\title{
Spectral asymptotics for infinite order pseudo-differential operators
}

\author{
Stevan Pilipović ${ }^{1} \cdot$ Bojan Prangoski $^{2}$. \\ Jasson Vindas ${ }^{3}$
}

Received: 24 September 2017 / Accepted: 15 December 2017 / Published online: 31 January 2018 (C) The Author(s) 2018. This article is an open access publication

\begin{abstract}
We study spectral properties of a class of global infinite order pseudodifferential operators and obtain the asymptotic behaviour of the spectral counting functions of such operators. Unlike their finite order counterparts, their spectral asymptotics are not of power-log-type but of log-type. The ultradistributional setting of such operators of infinite order makes the theory more complex so that the standard finite order global Weyl calculus cannot be used in this context.
\end{abstract}

Keywords Weyl asymptotic formula $\cdot$ Spectral asymptotics $\cdot$ Infinite order pseudodifferential operators $\cdot$ Hypoellipticity $\cdot$ Heat parametrix $\cdot$ Ultradistributions

Communicated by Ari Laptev.

The work of J. Vindas was supported by Ghent University, through the BOF-Grant 01N01014.

The work of S. Pilipovic was supported by the Project 174024 of the Serbian Ministry of EdSciTechDev.

Stevan Pilipović

stevan.pilipovic@dmi.uns.ac.rs

Bojan Prangoski

bprangoski@yahoo.com

Jasson Vindas

jasson.vindas@UGent.be

1 Department of Mathematics and Informatics, University of Novi Sad, Trg Dositeja Obradovića 4, 21000 Novi Sad, Serbia

2 Department of Mathematics, Faculty of Mechanical Engineering-Skopje, Karposh 2 b.b., 1000 Skopje, Macedonia

3 Department of Mathematics, Ghent University, Krijgslaan 281, 9000 Ghent, Belgium 
Mathematics Subject Classification 35P20 - 35S05 - 46F05 · 47D03

\section{Introduction}

In this article we study the spectral properties of global infinite order pseudodifferential operators. Our operator classes are intrinsically related to the ultradistributional framework so that the bounds on the derivatives of the symbols are controlled by Gevrey type weight sequences. Our aim is to establish Weyl asymptotic formulae for a large class of (hypoelliptic) $\Psi$ DOs of infinite order. It is worth mentioning that the Weyl asymptotics for the operators that we investigate here are not of power-logtype as in the finite order (distributional) setting, but of log-type, which in turn yields that the eigenvalues of infinite order $\Psi$ DOs, with appropriate assumptions, are "very sparse". As a by-product of our analysis, we also obtain Weyl asymptotic formulae for a class of finite order Shubin $\Psi$ DOs with some conditions on the symbols that are not the ones usually discussed in the literature.

The spaces of symbols and corresponding pseudo-differential operators involved in this work were introduced by Prangoski (see [18] for the symbolic calculus) and then extensively studied in several articles by himself and his coauthors; we refer to works of Cappiello [2,3] for similar symbol classes related to SG-hyperbolic problems of finite order. The definition of these symbols classes is linked to two Gevrey type weight sequences $A_{p}$ and $M_{p}, p \in \mathbb{N}$. The first one controls the smoothness, while the second one controls the growth at infinity of the symbols. These symbol classes are denoted by $\Gamma_{A_{p}, \rho}^{\left(M_{p}\right), \infty}$ and $\Gamma_{A_{p}, \rho}^{\left\{M_{p}\right\}, \infty}$. The first one gives rise to operators acting continuously on Gelfand-Shilov spaces of Beurling type (i.e. of $\left(M_{p}\right)$-class) and the second one on Gelfand-Shilov spaces of Roumieu type (of $\left\{M_{p}\right\}$-class); we will employ $\Gamma_{A_{p}, \rho}^{*, \infty}$ as a common notation for both cases. Since the symbols are allowed to grow sub-exponentially, i.e. ultrapolynomially, the corresponding $\Psi$ DOs are of infinite order and they go beyond the classical Weyl-Hörmander calculus.

The article is organised as follows. Section 2 gives some basic background material about the Gelfand-Shilov type spaces $\mathcal{S}^{*}\left(\mathbb{R}^{d}\right)$ and $\mathcal{S}^{*}\left(\mathbb{R}^{d}\right)$. We collect and explain in Sect. 3 some useful properties of the symbol classes $\Gamma_{A_{p}, \rho}^{*, \infty}$ and the corresponding global pseudo-differential operators. Further results related to the symbolic calculus that will be employed in the article are stated in the "Appendix" (Sect. 8).

Section 4 is devoted to establishing the semi-boundedness of the Weyl quantisation $a^{w}$ of a positive hypoelliptic infinite order symbol $a$. This will be achieved with the aid of results on anti-Wick quantisation from [16]. This result is interesting by itself because hypoellipticity in this setting allows the symbols to approach 0 subexponentially and thus generalises the familiar result for finite order operators. As a consequence, for hypoelliptic real-valued $a$ such that $|a(w)| \rightarrow \infty$ as $|w| \rightarrow \infty$, one obtains that the closure $\bar{A}$ of the unbounded operator $A$ on $L^{2}\left(\mathbb{R}^{d}\right)$ generated by $a^{w}$ is self-adjoint and has a spectrum given by a sequence of eigenvalues $\lambda_{n}, n \in \mathbb{N}$, tending to $\infty$ or $-\infty$, with eigenfunctions belonging to $\mathcal{S}^{*}\left(\mathbb{R}^{d}\right)$ and forming an orthonormal basis for $L^{2}\left(\mathbb{R}^{d}\right)$. 
We state in Sect. 5 our main results concerning Weyl asymptotic formulae and we postpone their proofs to Sect. 7, after developing the necessary machinery. We assume there that the symbol $a$ satisfies elliptic type bounds with respect to a rather general comparison function $f$ that is positive, increasing, and has suitable growth order. Theorem 5.1 gives the asymptotic behaviour of the spectral counting function $N(\lambda)$ for infinite order symbols, which corresponds to $f$ being of actual ultrapolynomial growth (and thus $f$ increases faster than any power function at $\infty$ ). Even more, our method yields new interesting results for Shubin type $\Psi$ DOs of finite order. Theorem 5.2 deals with the case of finite order Shubin type hypoelliptic symbols that satisfy elliptic bounds but with certain growth conditions on $f$ that appear to be different from the ones treated in the literature (cf. $[13,20]$ ). Theorem 5.4 provides an $O$-bound for $N(\lambda)$ by requiring only knowledge on a lower bound for the symbol. We present there also some illustrative examples.

The heat kernel analysis needed for the proofs of the Weyl asymptotic formulae for the class of operators under consideration is given in Sect. 6. We consider a realvalued hypoelliptic symbol $a$ in $\Gamma_{A_{p}, \rho}^{*, \infty}$ such that $a(w) / \ln |w| \rightarrow+\infty$ as $|w| \rightarrow \infty$. The main goal is the analysis of the semigroup $T(t) f=\sum_{j=0}^{\infty} e^{-t \lambda_{j}}\left(f, \varphi_{j}\right) \varphi_{j}$, $f \in L^{2}\left(\mathbb{R}^{d}\right), t \geq 0$, with infinitesimal generator $-\bar{A}$ [the closure of $-a^{w}$ in $\left.L^{2}\left(\mathbb{R}^{d}\right)\right]$ where $\lambda_{j}$ and $\varphi_{j}$ are the eigenvalues and eigenfunctions of $\bar{A}$. The crucial result to be shown here is that $T(t), t \geq 0$, form a smooth family of operators continuously acting on $\mathcal{S}^{*}\left(\mathbb{R}^{d}\right)$. The proofs of these facts are rather lengthy and we devote a whole subsection to them. It is important to stress that the classical approach does not work here (cf. Remark 6.14); one of the main reasons is the lack of Shubin-Sobolev spaces that fill in the gaps between the Gelfand-Shilov type spaces $\mathcal{S}^{*}\left(\mathbb{R}^{d}\right)$ and $L^{2}\left(\mathbb{R}^{d}\right)$, so we had to develop new techniques to overcome the problems. Once we have these properties of the semigroup $T(t), t \geq 0$, we prove that it is equal to the heat parametrix of $a^{w}$ as constructed in [17] modulo a smooth family of ultra-smoothing operators and use this to obtain the asymptotic formula

$$
\sum_{j=0}^{\infty} e^{-t \lambda_{j}}=\frac{1}{(2 \pi)^{d}} \int_{\mathbb{R}^{2 d}} e^{-t a(x, \xi)} d x d \xi+O\left(\int_{\mathbb{R}^{2 d}} \frac{e^{-\frac{t}{4} a(x, \xi)}}{\langle(x, \xi)\rangle^{2 \rho}} d x d \xi\right), \quad t \rightarrow 0^{+}
$$

This key asymptotic formula is the starting point for the proofs of our main theorems from Sect. 5 concerning Weyl asymptotic formulae; such proofs are the content of Sect. 7. The passage from asymptotics of the heat semigroup to Weyl formulae is accomplished using ideas from the theory of regular variation $[1,11]$ and Tauberian tools.

\section{Preliminaries}

For $x \in \mathbb{R}^{d}$ and $\alpha \in \mathbb{N}^{d}$, we will use the notation $\langle x\rangle=\left(1+|x|^{2}\right)^{1 / 2}, D^{\alpha}=$ $D_{1}^{\alpha_{1}} \ldots D_{d}^{\alpha_{d}}$, where $D_{j}^{\alpha_{j}}=i^{-\alpha_{j}} \partial^{\alpha_{j}} / \partial x_{j}{ }^{\alpha_{j}}$. Following Komatsu [8], we work with some of the standard conditions $(M .1),(M .2),(M .3),(M .3)^{\prime}$ and $(M .4)$ on sequences 
of positive numbers $M_{p}, p \in \mathbb{N}$, for which we always assume $M_{0}=1$. We only recall (M.4):

(M.4) $M_{p}^{2} / p !^{2} \leq\left(M_{p-1} /(p-1) !\right) \cdot\left(M_{p+1} /(p+1) !\right), p \in \mathbb{Z}_{+}$.

Note that the Gevrey sequence $M_{p}=p !^{s}, s>1$, satisfies all the conditions listed above. Given two weight sequences $M_{p}$ and $\tilde{M}_{p}$, the notation $M_{p} \subset \tilde{M}_{p}$ (resp. $M_{p} \prec \tilde{M}_{p}$ ) means that there are $C, L>0$ (resp. for every $L>0$ there is $C>0$ ) such that $M_{p} \leq C L^{p} \tilde{M}_{p}, \forall p \in \mathbb{N}$. For a multi-index $\alpha \in \mathbb{N}^{d}, M_{\alpha}$ stands for $M_{|\alpha|},|\alpha|=$ $\alpha_{1}+\cdots+\alpha_{d}$. As usual [8, Section 3], we set $m_{p}=M_{p} / M_{p-1}, p \in \mathbb{Z}_{+}$, and if $M_{p}$ satisfies $(M .1)$ and $M_{p} / C^{p} \rightarrow \infty$, for any $C>0$ (which obviously holds when $M_{p}$ satisfies $\left.(M .3)^{\prime}\right)$, its associated function is defined by $M(\rho)=\sup _{p \in \mathbb{N}} \ln _{+} \rho^{p} / M_{p}$, $\rho>0$. It is a non-negative, continuous, monotonically increasing function, vanishes for sufficiently small $\rho>0$, and increases more rapidly than $\ln \rho^{n}$ as $\rho \rightarrow \infty$, for any $n \in \mathbb{N}$. When $M_{p}=p !^{s}$, with $s>0$, we have $M(\rho) \asymp \rho^{1 / s}$.

For a regular compact set $K \subseteq \mathbb{R}^{d}$ (i.e. $K=\overline{\operatorname{int} K}$ ) and $h>0, \mathcal{E}^{M_{p}, h}(K)$ is the Banach space (abbreviated as $(B)$-space) of all $\varphi \in C^{\infty}$ (int $K$ ) whose derivatives extend to continuous functions on $K$ and satisfy $\sup _{\alpha \in \mathbb{N}^{d}} \sup _{x \in K}\left|D^{\alpha} \varphi(x)\right| /\left(h^{\alpha} M_{\alpha}\right)<$ $\infty$ and $\mathcal{D}_{K}^{M_{p}, h}$ denotes its subspace of all smooth functions supported by $K$. For $U \subseteq$ $\mathbb{R}^{d}$, we define as locally convex spaces (abbreviated as 1.c.s.) $\mathcal{E}^{\left(M_{p}\right)}(U), \mathcal{E}^{\left\{M_{p}\right\}}(U)$, $\mathcal{D}^{\left(M_{p}\right)}(U), \mathcal{D}^{\left\{M_{p}\right\}}(U)$ and their strong duals, the corresponding spaces of ultradistributions of Beurling and Roumieu type, cf. [8-10].

We denote by $\Re$ the set of all positive sequences which monotonically increase to infinity. There is a natural order on $\Re$ defined by $\left(r_{p}\right) \leq\left(k_{p}\right)$ if $r_{p} \leq k_{p}, \forall p \in \mathbb{Z}_{+}$, and with it $(\mathfrak{R}, \leq)$ becomes a directed set. For $\left(r_{p}\right) \in \mathfrak{R}$, consider the sequence $N_{0}=1$, $N_{p}=M_{p} \prod_{j=1}^{p} r_{j}, p \in \mathbb{Z}_{+}$. It is easy to check that this sequence satisfies $(M .1)$ and $(M .3)^{\prime}$ when $M_{p}$ does so and its associated function will be denoted by $N_{r_{p}}(\rho)$, i.e. $N_{r_{p}}(\rho)=\sup _{p \in \mathbb{N}} \ln _{+} \rho^{p} /\left(M_{p} \prod_{j=1}^{p} r_{j}\right), \rho>0$. Note that for $\left(r_{p}\right) \in \Re$ and $k>0$ there is $\rho_{0}>0$ such that $N_{r_{p}}(\rho) \leq M(k \rho)$, for $\rho>\rho_{0}$.

A measurable function $f$ on $\mathbb{R}^{d}$ is said to have ultrapolynomial growth of class $\left(M_{p}\right)$ (resp. of class $\left\{M_{p}\right\}$ ) if $\left\|e^{-M(h|\cdot|)} f\right\|_{L^{\infty}\left(\mathbb{R}^{d}\right)}<\infty$ for some $h>0$ (resp. for every $h>0$ ). We have the following equivalent description of continuous functions of ultrapolynomial growth of class $\left\{M_{p}\right\}$.

Lemma 2.1 [17, Lemma 2.1] Let $B \subseteq C\left(\mathbb{R}^{d}\right)$. The following conditions are equivalent: (i) For everyh $>0$ there exists $C>0$ such that $|f(x)| \leq C e^{M(h|x|)}$, for all $x \in \mathbb{R}^{d}, f \in B$; (ii) There exist $\left(r_{p}\right) \in \mathfrak{R}$ and $C>0$ such that $|f(x)| \leq C e^{N_{r_{p}}(|x|)}$, for all $x \in \mathbb{R}^{d}, f \in B$.

If $M_{p}$ satisfies $(M .1)$ and $(M .3)^{\prime}$, for $m>0$ we denote by $\mathcal{S}_{\infty}^{M_{p}, m}\left(\mathbb{R}^{d}\right)$ the $(B)$ space of all $\varphi \in C^{\infty}\left(\mathbb{R}^{d}\right)$ for which the norm $\sup _{\alpha \in \mathbb{N}^{d}} m^{|\alpha|}\left\|e^{M(m|\cdot|)} D^{\alpha} \varphi\right\|_{L^{\infty}\left(\mathbb{R}^{d}\right)} / M_{\alpha}$ is finite. The spaces of sub-exponentially decreasing ultradifferentiable function of Beurling and Roumieu type are defined as 


$$
\mathcal{S}^{\left(M_{p}\right)}\left(\mathbb{R}^{d}\right)=\lim _{m \rightarrow \infty} \mathcal{S}_{\infty}^{M_{p}, m}\left(\mathbb{R}^{d}\right) \text { and } \mathcal{S}^{\left\{M_{p}\right\}}\left(\mathbb{R}^{d}\right)=\underset{m \rightarrow 0}{\lim _{m \rightarrow 0}} \mathcal{S}_{\infty}^{M_{p}, m}\left(\mathbb{R}^{d}\right),
$$

respectively. Their strong duals $\mathcal{S}^{\prime\left(M_{p}\right)}\left(\mathbb{R}^{d}\right)$ and $\mathcal{S}^{\prime\left\{M_{p}\right\}}\left(\mathbb{R}^{d}\right)$ are the spaces of tempered ultradistributions of Beurling and Roumieu type, respectively. When $M_{p}=p !^{s}, s>1$, $\mathcal{S}^{\left\{M_{p}\right\}}\left(\mathbb{R}^{d}\right)$ is just the Gelfand-Shilov space $\mathcal{S}_{s}^{s}\left(\mathbb{R}^{d}\right)$ [13]. If $M_{p}$ satisfies $(M .2)$, the ultradifferential operators of class $*$ act continuously on $\mathcal{S}^{*}\left(\mathbb{R}^{d}\right)$ and $\mathcal{S}^{*}\left(\mathbb{R}^{d}\right.$ ) (for the definition of ultradifferential operators see [8]). These spaces are nuclear and the Fourier transform is a topological isomorphism on them. We refer to $[6,15]$ for the topological properties of $\mathcal{S}^{*}\left(\mathbb{R}^{d}\right)$ and $\mathcal{S}^{*}\left(\mathbb{R}^{d}\right)$. Here we recall that, when $M_{p}$ satisfies (M.2), the space $\mathcal{S}^{\left\{M_{p}\right\}}\left(\mathbb{R}^{d}\right)$ is topologically isomorphic to $\lim _{\left(r_{p}\right) \in \mathfrak{R}} \mathcal{S}_{\infty}^{M_{p},\left(r_{p}\right)}\left(\mathbb{R}^{d}\right)$, where the projective limit is taken with respect to the natural order on $\mathfrak{R}$ defined above and $\mathcal{S}_{\infty}^{M_{p},\left(r_{p}\right)}\left(\mathbb{R}^{d}\right)$ is the $(B)$-space of all $\varphi \in C^{\infty}\left(\mathbb{R}^{d}\right)$ for which the norm $\sup _{\alpha \in \mathbb{N}^{d}}\left\|e^{N_{r_{p}}(|\cdot|)} D^{\alpha} \varphi\right\|_{L^{\infty}\left(\mathbb{R}^{d}\right)} /\left(M_{\alpha} \prod_{j=1}^{|\alpha|} r_{j}\right)$ is finite.

Next, let $E$ and $F$ be 1.c.s.; $\mathcal{L}(E, F)$ stands for the space of continuous linear mappings from $E$ to $F$; when $E=F$, we write $\mathcal{L}(E)$. We employ the notation $\mathcal{L}_{b}(E, F)$ for the space $\mathcal{L}(E, F)$ equipped with the topology of bounded convergence and, similarly, $\mathcal{L}_{p}(E, F)$ and $\mathcal{L}_{\sigma}(E, F)$ stand for $\mathcal{L}(E, F)$ equipped with the topologies of precompact and simple convergence, respectively. Furthermore, $E \hookrightarrow F$ means that $E$ is continuously and densely included in $F$. For $(a, b) \subseteq \mathbb{R}$ and $0 \leq k \leq \infty$, $C^{k}((a, b) ; E)$ stands for the vector space of $k$ times continuously differentiable $E$ valued functions on $(a, b)$, while $C^{k}([a, b) ; E)$ for the space of those on $[a, b)$, where the derivatives at $a$ are to be understood as right derivatives; we use analogous notations when considering functions over $(a, b]$ or $[a, b]$.

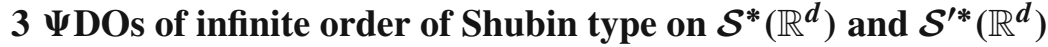

We discuss in this section properties of the classes of infinite order $\Psi$ DOs that we shall consider in the article; see also the "Appendix" for other important facts about their symbolic calculus. We refer to [4,18] and [17, Sections 3 and 4] for complete accounts.

\subsection{Symbol classes and symbolic calculus}

Let $A_{p}$ and $M_{p}$ be two weight sequences of positive numbers such that $A_{0}=A_{1}=$ $M_{0}=M_{1}=1$. We assume that $M_{p}$ satisfies (M.1), (M.2) and (M.3), and that $A_{p}$ satisfies (M.1), (M.2), (M.3) $)^{\prime}$ and (M.4). Of course, we may assume that the constants $c_{0}$ and $H$ appearing in (M.2) are the same for both sequences $M_{p}$ and $A_{p}$. We assume that $A_{p} \subset M_{p}$. Let $\rho_{0}=\inf \left\{\rho \in \mathbb{R}_{+} \mid A_{p} \subset M_{p}^{\rho}\right\}$; clearly $0<\rho_{0} \leq 1$. Throughout the rest of the article, $\rho$ is a fixed number satisfying $\rho_{0} \leq \rho \leq 1$, if the infimum is reached, or, otherwise $\rho_{0}<\rho \leq 1$. Clearly, we may also assume that $A_{p} \leq c_{0} L^{p} M_{p}^{\rho}$, where $c_{0} \geq 1$ is the constant from (M.2). 
For $h, m>0$, define (following [18]) $\Gamma_{A_{p}, \rho}^{M_{p}, \infty}\left(\mathbb{R}^{2 d} ; h, m\right)$ to be the $(B)$-space of all $a \in C^{\infty}\left(\mathbb{R}^{2 d}\right)$ for which the norm

$$
\sup _{\alpha, \beta \in \mathbb{N}^{d}} \sup _{(x, \xi) \in \mathbb{R}^{2 d}} \frac{\left|D_{\xi}^{\alpha} D_{x}^{\beta} a(x, \xi)\right|\langle(x, \xi)\rangle^{\rho|\alpha|+\rho|\beta|} e^{-M(m|\xi|)} e^{-M(m|x|)}}{h^{|\alpha|+|\beta|} A_{\alpha} A_{\beta}} .
$$

is finite. As 1.c.s., we define

$$
\begin{array}{ll}
\Gamma_{A_{p}, \rho}^{\left(M_{p}\right), \infty}\left(\mathbb{R}^{2 d} ; m\right)=\underset{h \rightarrow 0}{\lim } \Gamma_{A_{p}, \rho}^{M_{p}, \infty}\left(\mathbb{R}^{2 d} ; h, m\right) ; & \Gamma_{A_{p}, \rho}^{\left(M_{p}\right), \infty}\left(\mathbb{R}^{2 d}\right) \underset{m \rightarrow \infty}{\lim } \Gamma_{A_{p}, \rho}^{\left(M_{p}\right), \infty}\left(\mathbb{R}^{2 d} ; m\right) ; \\
\Gamma_{A_{p}, \rho}^{\left\{M_{p}\right\}, \infty}\left(\mathbb{R}^{2 d} ; h\right)=\underset{m \rightarrow 0}{\lim } \Gamma_{A_{p}, \rho}^{M_{p}, \infty}\left(\mathbb{R}^{2 d} ; h, m\right) ; & \Gamma_{A_{p}, \rho}^{\left\{M_{p}\right\}, \infty}\left(\mathbb{R}^{2 d}\right)=\underset{h \rightarrow \infty}{\lim } \Gamma_{A_{p}, \rho}^{\left\{M_{p}\right\}, \infty}\left(\mathbb{R}^{2 d} ; h\right) .
\end{array}
$$

Then, $\Gamma_{A_{p}, \rho}^{\left(M_{p}\right), \infty}\left(\mathbb{R}^{2 d} ; m\right)$ and $\Gamma_{A_{p}, \rho}^{\left\{M_{p}\right\}, \infty}\left(\mathbb{R}^{2 d} ; h\right)$ are $(F)$-spaces. The spaces $\Gamma_{A_{p}, \rho}^{*, \infty}\left(\mathbb{R}^{2 d}\right)$ are barrelled and bornological.

For $\tau \in \mathbb{R}$ and $a \in \Gamma_{A_{p}, \rho}^{*, \infty}\left(\mathbb{R}^{2 d}\right)$, the $\tau$-quantisation of $a$ is the operator $\operatorname{Op}_{\tau}(a)$, continuous on $\mathcal{S}^{*}\left(\mathbb{R}^{d}\right)$ given by the iterated integral:

$$
\left(\mathrm{Op}_{\tau}(a) u\right)(x)=\frac{1}{(2 \pi)^{d}} \int_{\mathbb{R}^{d}} \int_{\mathbb{R}^{d}} e^{i(x-y) \xi} a((1-\tau) x+\tau y, \xi) u(y) d y d \xi
$$

Let $t \geq 0$. We denote $Q_{t}=\left\{(x, \xi) \in \mathbb{R}^{2 d} \mid\langle x\rangle<t,\langle\xi\rangle<t\right\}$ and $Q_{t}^{c}=\mathbb{R}^{2 d} \backslash Q_{t}$. If $0 \leq t \leq 1$, then $Q_{t}=\emptyset$ and $Q_{t}^{c}=\mathbb{R}^{2 d}$. Let $B \geq 0$ and $h, m>0$. Following [17,18], denote by $F S_{A_{p}, \rho}^{M_{p}, \infty}\left(\mathbb{R}^{2 d} ; B, h, m\right)$ the vector space of all formal series $\sum_{j=0}^{\infty} a_{j}(x, \xi)$ such that $a_{j} \in C^{\infty}$ (int $\left.Q_{B m_{j}}^{c}\right), D_{\xi}^{\alpha} D_{x}^{\beta} a_{j}(x, \xi)$ can be extended to a continuous function on $Q_{B m_{j}}^{c}$ for all $\alpha, \beta \in \mathbb{N}^{d}$ and

$\sup _{j \in \mathbb{N}} \sup _{\alpha, \beta} \sup _{(x, \xi) \in Q_{B m_{j}}^{c}} \frac{\left|D_{\xi}^{\alpha} D_{x}^{\beta} a_{j}(x, \xi)\right|\langle(x, \xi)\rangle^{\rho|\alpha|+\rho|\beta|+2 j \rho} e^{-M(m|\xi|)} e^{-M(m|x|)}}{h^{|\alpha|+|\beta|+2 j} A_{\alpha} A_{\beta} A_{j} A_{j}}<\infty$.

In the above, we use the convention $m_{0}=0$ and hence, $Q_{B m_{0}}^{c}=\mathbb{R}^{2 d}$. With this norm, $F S_{A_{p}, \rho}^{M_{p}, \infty}\left(\mathbb{R}^{2 d} ; B, h, m\right)$ becomes a $(B)$-space. As l.c.s., we define

$$
\begin{aligned}
F S_{A_{p}, \rho}^{\left(M_{p}\right), \infty}\left(\mathbb{R}^{2 d} ; B, m\right) & =\lim _{h \rightarrow 0} F S_{A_{p}, \rho}^{M_{p}, \infty}\left(\mathbb{R}^{2 d} ; B, h, m\right), \\
F S_{A_{p}, \rho}^{\left(M_{p}\right), \infty}\left(\mathbb{R}^{2 d} ; B\right) & =\underset{m \rightarrow \infty}{\lim } F S_{A_{p}, \rho}^{\left(M_{p}\right), \infty}\left(\mathbb{R}^{2 d} ; B, m\right),
\end{aligned}
$$




$$
\begin{gathered}
F S_{A_{p}, \rho}^{\left\{M_{p}\right\}, \infty}\left(\mathbb{R}^{2 d} ; B, h\right)=\lim _{m \rightarrow 0} F S_{A_{p}, \rho}^{M_{p}, \infty}\left(\mathbb{R}^{2 d} ; B, h, m\right), \\
F S_{A_{p}, \rho}^{\left\{M_{p}\right\}, \infty}\left(\mathbb{R}^{2 d} ; B\right)= \\
\underset{h \rightarrow \infty}{\lim } F S_{A_{p}, \rho}^{\left\{M_{p}\right\}, \infty}\left(\mathbb{R}^{2 d} ; B, h\right) .
\end{gathered}
$$

Then, the spaces $F S_{A_{p}, \rho}^{\left(M_{p}\right), \infty}\left(\mathbb{R}^{2 d} ; B, m\right)$ and $F S_{A_{p}, \rho}^{\left\{M_{p}\right\}, \infty}\left(\mathbb{R}^{2 d} ; B, h\right)$ are $(F)$-spaces and the space $F S_{A_{p}, \rho}^{*, \infty}\left(\mathbb{R}^{2 d} ; B\right)$ is barrelled and bornological. The inclusion mapping $\Gamma_{A_{p}, \rho}^{*, \infty}\left(\mathbb{R}^{2 d}\right) \rightarrow F S_{A_{p}, \rho}^{*, \infty}\left(\mathbb{R}^{2 d} ; B\right)$, defined as $a \mapsto \sum_{j \in \mathbb{N}} a_{j}$, where $a_{0}=a$ and $a_{j}=0$, $j \geq 1$, is continuous. We call this inclusion the canonical one. For $B_{1} \leq B_{2}$, the map$\operatorname{ping} \sum_{j} p_{j} \mapsto \sum_{j} p_{\left.j\right|_{Q_{B_{2} m_{j}}^{c}}}, F S_{A_{p}, \rho}^{*, \infty}\left(\mathbb{R}^{2 d} ; B_{1}\right) \rightarrow F S_{A_{p}, \rho}^{*, \infty}\left(\mathbb{R}^{2 d} ; B_{2}\right)$ is continuous. We also call this mapping canonical.

Let $F S_{A_{p}, \rho}^{*, \infty}\left(\mathbb{R}^{2 d}\right)=\underset{B \rightarrow \infty}{\lim _{\longrightarrow} F S_{A_{p}, \rho}^{*, \infty}}\left(\mathbb{R}^{2 d} ; B\right)$, where the inductive limit is taken in an algebraic sense and the linking mappings are the canonical ones described above. Clearly, $F S_{A_{p}, \rho}^{*, \infty}\left(\mathbb{R}^{2 d}\right)$ is non-trivial.

If $\sum_{j} a_{j} \in F S_{A_{p}, \rho}^{*, \infty}\left(\mathbb{R}^{2 d} ; B\right)$ and $n \in \mathbb{N},\left(\sum_{j} a_{j}\right)_{n}$ will just mean the function $a_{n} \in C^{\infty}\left(Q_{B m_{n}}^{c}\right)$, while $\left(\sum_{j} a_{j}\right)_{<n}$ denotes the function $\sum_{j=0}^{n-1} a_{j} \in C^{\infty}\left(Q_{B m_{n-1}}^{c}\right)$. Furthermore, 1 denotes the element $\sum_{j} a_{j} \in F S_{A_{p}, \rho}^{*, \infty}\left(\mathbb{R}^{2 d} ; B\right)$ given by $a_{0}(x, \xi)=1$ and $a_{j}(x, \xi)=0, j \in \mathbb{Z}_{+}$.

Recall, Prangoski [18, Definition 3] that two sums, $\sum_{j \in \mathbb{N}} a_{j}, \sum_{j \in \mathbb{N}} b_{j} \in$ $F S_{A_{p}, \rho}^{*, \infty}\left(\mathbb{R}^{2 d}\right)$, are said to be equivalent, in notation $\sum_{j \in \mathbb{N}} a_{j} \sim \sum_{j \in \mathbb{N}} b_{j}$, if there exist $m>0$ and $B>0$ (resp. there exist $h>0$ and $B>0$ ), such that for every $h>0$ (resp. for every $m>0$ ),

$\sup _{n \in \mathbb{Z}_{+}} \sup _{\alpha, \beta} \sup _{(x, \xi) \in Q_{B m_{n}}^{c}} \frac{\left|D_{\xi}^{\alpha} D_{x}^{\beta} \sum_{j<n}\left(a_{j}(x, \xi)-b_{j}(x, \xi)\right)\right|\langle(x, \xi)\rangle^{\rho|\alpha|+\rho|\beta|+2 n \rho}}{h^{|\alpha|+|\beta|+2 n} A_{\alpha} A_{\beta} A_{n} A_{n} e^{M(m|\xi|)} e^{M(m|x|)}}<\infty$.

\subsection{Subordination}

In the sequel, we will often use the notation $w=(x, \xi) \in \mathbb{R}^{2 d}$.

Let $\Lambda$ be an index set and $\left\{f_{\lambda} \mid \lambda \in \Lambda\right.$ be a set of positive continuous functions on $\mathbb{R}^{2 d}$ each with ultrapolynomial growth of class $*$. We say that a set $U^{(\Lambda)}=\left\{\sum_{j} a_{j}^{(\lambda)} \mid \lambda \in \Lambda\right\} \subseteq F S_{A_{p}, \rho}^{*, \infty}\left(\mathbb{R}^{2 d} ; B^{\prime}\right)$ is subordinated to $\left\{f_{\lambda} \mid \lambda \in \Lambda\right\}$ in $F S_{A_{p}, \rho}^{*, \infty}\left(\mathbb{R}^{2 d}\right)$, in notation $U^{(\Lambda)} \precsim\left\{f_{\lambda} \mid \lambda \in \Lambda\right\}$, if the following estimate holds: there exists $B \geq B^{\prime}$ such that for every $h>0$ there exists $C>0$ (resp. there exist $h, C>0$ ) such that

$$
\sup _{\lambda \in \Lambda} \sup _{j \in \mathbb{N}} \sup _{\alpha \in \mathbb{N}^{2 d}} \sup _{w \in Q_{B m_{j}}^{c}} \frac{\left|D_{w}^{\alpha} a_{j}^{(\lambda)}(w)\right|\langle w\rangle^{\rho(|\alpha|+2 j)}}{h^{|\alpha|+2 j} A_{|\alpha|+2 j} f_{\lambda}(w)} \leq C .
$$


Whenever we want to emphasise that the estimate is valid for a particular $B \geq B^{\prime}$, we write $U^{(\Lambda)} \precsim\left\{f_{\lambda} \mid \lambda \in \Lambda\right\}$ in $F S_{A_{p}, \rho}^{*, \infty}\left(\mathbb{R}^{2 d} ; B\right)$. When $f_{\lambda}=f, \forall \lambda \in \Lambda$, we abbreviate the notation and simply write $U \precsim f$, and then say that $U$ is subordinated to $f$. Clearly, for $U \subseteq F S_{A_{p}, \rho}^{*, \infty}\left(\mathbb{R}^{2 d} ; B_{1}\right)$ such that $U \precsim f$, there exists $B \geq B_{1}$ such that the image of $U$ under the canonical mapping $F S_{A_{p}, \rho}^{*, \infty}\left(\mathbb{R}^{2 d} ; B_{1}\right) \rightarrow F S_{A_{p}, \rho}^{*, \infty}\left(\mathbb{R}^{2 d} ; B\right)$ is a bounded subset of $F S_{A_{p}, \rho}^{\left(M_{p}\right), \infty}\left(\mathbb{R}^{2 d} ; B, m\right)$ for some $m>0$ (resp. a bounded subset of $F S_{A_{p}, \rho}^{\left\{M_{p}\right\}, \infty}\left(\mathbb{R}^{2 d} ; B, h\right)$ for some $\left.h>0\right)$. For such $U$, we say that a bounded set $V$ in $\Gamma_{A_{p}, \rho}^{\left(M_{p}\right), \infty}\left(\mathbb{R}^{2 d} ; m\right)$ for some $m>0$ (resp. in $\Gamma_{A_{p}, \rho}^{\left\{M_{p}\right\}, \infty}\left(\mathbb{R}^{2 d} ; h\right)$ for some $h>0$ ) is subordinated to $U$ under $f$, in notations $V \precsim f U$, if there exists a surjective mapping $\Sigma: U \rightarrow V$ such that the following estimate holds: there exists $B \geq B_{1}$ such that for every $h>0$ there exists $C>0$ (resp. there exist $h, C>0$ ) such that for all $\sum_{j} a_{j} \in U$ and the corresponding $\Sigma\left(\sum_{j} a_{j}\right)=a \in V$

$$
\sup _{n \in \mathbb{Z}_{+}} \sup _{\alpha \in \mathbb{N}^{2} d} \sup _{w \in Q_{B m_{n}}^{c}} \frac{\left|D_{w}^{\alpha}\left(a(w)-\sum_{j<n} a_{j}(w)\right)\right|\langle w\rangle^{\rho(|\alpha|+2 n)}}{h^{|\alpha|+2 n} A_{|\alpha|+2 n} f(w)} \leq C .
$$

Again, when we want to emphasise the particular $B$ for which this holds, we write $V \precsim_{f} U$ in $F S_{A_{p}, \rho}^{*, \infty}\left(\mathbb{R}^{2 d} ; B\right)$. If $V \precsim_{f} U$ and if we denote by $\tilde{V}$ the image of $V$ under the canonical inclusion $\Gamma_{A_{p}, \rho}^{*, \infty}\left(\mathbb{R}^{2 d}\right) \rightarrow F S_{A_{p}, \rho}^{*, \infty}\left(\mathbb{R}^{2 d} ; 0\right), a \mapsto a+\sum_{j \in \mathbb{Z}_{+}} 0$, then by specialising the above estimate for $n=1$ together with the boundedness of $V$ in $\Gamma_{A_{p}, \rho}^{\left(M_{p}\right), \infty}\left(\mathbb{R}^{2 d} ; m\right)$ for some $m>0$ (resp. in $\Gamma_{A_{p}, \rho}^{\left\{M_{p}\right\}, \infty}\left(\mathbb{R}^{2 d} ; h\right)$ for some $\left.h>0\right)$ and the continuity and positivity of $f$, we derive that $\tilde{V} \precsim f$ in $F S_{A_{p}, \rho}^{*, \infty}\left(\mathbb{R}^{2 d} ; 0\right)$. In such a case, we slightly abuse notation and write $V \precsim f$. This estimate also implies $\Sigma\left(\sum_{j} a_{j}\right) \sim$ $\sum_{j} a_{j}$. To see that given such an $U \subseteq F S_{A_{p}, \rho}^{*, \infty}\left(\mathbb{R}^{2 d} ; B\right)$ there always exists $V \precsim f U$, we can proceed as follows. Let $\psi \in \mathcal{D}^{\left(A_{p}\right)}\left(\mathbb{R}^{d}\right)$ in the $\left(M_{p}\right)$ case and $\psi \in \mathcal{D}^{\left\{A_{p}\right\}}\left(\mathbb{R}^{d}\right)$ in the $\left\{M_{p}\right\}$ case respectively, such that $0 \leq \psi \leq 1, \psi(\xi)=1$ when $\langle\xi\rangle \leq 2$ and $\psi(\xi)=0$ when $\langle\xi\rangle \geq 3$. Set $\chi(x, \xi)=\psi(x) \psi(\xi), \chi_{n, R}(w)=\chi\left(w /\left(R m_{n}\right)\right)$ for $n \in \mathbb{Z}_{+}$and $R>0$ and put $\chi_{0, R}(w)=0$. Given $U \subseteq F S_{A_{p}, \rho}^{*, \infty}\left(\mathbb{R}^{2 d} ; B\right)$ as above, for $\sum_{j} a_{j} \in U$ denote $R\left(\sum_{j} a_{j}\right)(w)=\sum_{j=0}^{\infty}\left(1-\chi_{j, R}(w)\right) a_{j}(w)$. If $R>B$, this is a well defined smooth function on $\mathbb{R}^{2 d}$, since the series is locally finite.

Proposition 3.1 [17, Proposition 3.3] Let $U=\left\{\sum_{j} a_{j}^{(\lambda)} \mid \lambda \in \Lambda\right\}$ be a subset of $F S_{A_{p}, \rho}^{*, \infty}\left(\mathbb{R}^{2 d} ; B^{\prime}\right)$ that is subordinated to $\left\{f_{\lambda} \mid \lambda \in \Lambda\right\}$ in $F S_{A_{p}, \rho}^{*, \infty}\left(\mathbb{R}^{2 d}\right)$. There exists $R_{0}>B^{\prime}$ such that for each $R \geq R_{0}, U_{R}=\left\{R\left(\sum_{j} a_{j}^{(\lambda)}\right) \mid \lambda \in \Lambda\right\} \subseteq \Gamma_{A_{p}, \rho}^{*, \infty}\left(\mathbb{R}^{2 d}\right)$ and the following estimate holds: there exists $B=B(R) \geq B^{\prime}$ such that for every $h>0$ there exists $C>0$ (resp. there exist $h, C>0$ ) such that

$\sup _{\lambda \in \Lambda} \sup _{n \in \mathbb{Z}_{+}} \sup _{\alpha \in \mathbb{N}^{2} d} \sup _{w \in Q_{B m_{n}}^{c}} \frac{\left|D_{w}^{\alpha}\left(R\left(\sum_{j} a_{j}^{(\lambda)}\right)(w)-\sum_{j<n} a_{j}^{(\lambda)}(w)\right)\right|\langle w\rangle^{\rho(|\alpha|+2 n)}}{h^{|\alpha|+2 n} A_{|\alpha|+2 n} f_{\lambda}(w)} \leq C$. 
If in addition $f_{\lambda}=f, \forall \lambda \in \Lambda$, then $U_{R}$ is bounded in $\Gamma_{A_{p}, \rho}^{\left(M_{p}\right), \infty}\left(\mathbb{R}^{2 d} ; m\right)$ for some $m>0$ (resp. bounded in $\Gamma_{A_{p}, \rho}^{\left\{M_{p}\right\}, \infty}\left(\mathbb{R}^{2 d} ; h\right)$ for some $h>0$ ) and hence $U_{R} \precsim f U$.

We say that this $U_{R}$ is canonically obtained from $U$ by $\left\{\chi_{n, R}\right\}_{n \in \mathbb{N}}$. Of course, here the mapping $\Sigma: U \rightarrow U_{R}$ is just $\sum_{j} a_{j} \mapsto R\left(\sum_{j} a_{j}\right)$.

Proposition 3.2 [17, Proposition 3.4] Let V be a bounded subset of $\Gamma_{A_{p}, \rho}^{\left(M_{p}\right), \infty}\left(\mathbb{R}^{2 d} ; \tilde{m}\right)$ for some $\tilde{m}>0$ (resp. of $\Gamma_{A_{p}, \rho}^{\left\{M_{p}\right\}, \infty}\left(\mathbb{R}^{2 d} ; \tilde{h}\right)$ for some $\left.\tilde{h}>0\right)$. Assume that there exist $B, m>0$ such that for every $h>0$ there exists $C>0$ (resp. there exist $B, h>0$ such that for every $m>0$ there exists $C>0$ ) such that

$$
\sup _{a \in V} \sup _{n \in \mathbb{Z}_{+}} \sup _{\alpha \in \mathbb{N}^{2} d} \sup _{w \in Q_{B m_{n}}^{c}} \frac{\left|D_{w}^{\alpha} a(w)\right|\langle w\rangle^{\rho(|\alpha|+2 n)}}{h^{|\alpha|+2 n} A_{|\alpha|+2 n} e^{M(m|w|)}} \leq C .
$$

Then, $\left\{\mathrm{Op}_{\tau}(a) \mid a \in U\right\}$ is an equicontinuous subset of $\mathcal{L}\left(\mathcal{S}^{\prime *}\left(\mathbb{R}^{d}\right), \mathcal{S}^{*}\left(\mathbb{R}^{d}\right)\right)$ for each $\tau \in \mathbb{R}$.

In what follows, we will frequently use the term "*-regularising set" for a subset of $\mathcal{L}\left(\mathcal{S}^{*}\left(\mathbb{R}^{d}\right), \mathcal{S}^{*}\left(\mathbb{R}^{d}\right)\right)$. Changing the quantisation and taking composition of $\Psi$ DOs with symbols in $\Gamma_{A_{p}, \rho}^{*, \infty}\left(\mathbb{R}^{2 d}\right)$ always results in $\Psi$ DOs with symbols in the same class modulo $*$-regularising operators; we collect some of these facts in the "Appendix" and we refer to $[17,18]$ for the complete theory.

\subsection{Weyl quantisation. The sharp product in $F S_{A_{p}, \rho}^{*, \infty}\left(\mathbb{R}^{2 d} ; B\right)$}

We recall in this and the next subsection results from [17] about the Weyl quantisation of symbols; we often write $a^{w}$ instead of $\mathrm{Op}_{1 / 2}(a)$.

Given $\sum_{j} a_{j}, \sum_{j} b_{j} \in F S_{A_{p}, \rho}^{*, \infty}\left(\mathbb{R}^{2 d} ; B\right)$ we define their sharp product, denoted as $\sum_{j} a_{j} \# \sum_{j} b_{j}$, via the formal series $\sum_{j} c_{j}=\sum_{j} a_{j} \# \sum_{j} b_{j}$ where

$$
c_{j}(x, \xi)=\sum_{s+k+l=j} \sum_{|\alpha+\beta|=l} \frac{(-1)^{|\beta|}}{\alpha ! \beta ! 2^{l}} \partial_{\xi}^{\alpha} D_{x}^{\beta} a_{s}(x, \xi) \partial_{\xi}^{\beta} D_{x}^{\alpha} b_{k}(x, \xi), \quad(x, \xi) \in Q_{B m_{j}}^{c} .
$$

It is easy to verify that $\sum_{j} c_{j}$ is a well defined element of $F S_{A_{p}, \rho}^{*, \infty}\left(\mathbb{R}^{2 d} ; B\right)$. If $a \in$ $\Gamma_{A_{p}, \rho}^{*, \infty}\left(\mathbb{R}^{2 d}\right)$, then $a \# \sum_{j} b_{j}$ will denote the \# product of the image of $a$ under the canonical inclusion $\Gamma_{A_{p}, \rho}^{*, \infty}\left(\mathbb{R}^{2 d}\right) \rightarrow F S_{A_{p}, \rho}^{*, \infty}\left(\mathbb{R}^{2 d} ; B\right)$ and $\sum_{j} b_{j}$. The same convention applies if $b \in \Gamma_{A_{p}, \rho}^{*, \infty}\left(\mathbb{R}^{2 d}\right)$ or if both $a, b \in \Gamma_{A_{p}, \rho}^{*, \infty}\left(\mathbb{R}^{2 d}\right)$.

Remark 3.3 If $\sum_{j} a_{j}, \sum_{j} b_{j} \in F S_{A_{p}, \rho}^{*, \infty}\left(\mathbb{R}^{2 d} ; B\right)$ and $\sum_{j} c_{j}=\sum_{j} a_{j} \# \sum_{j} b_{j}$, then $\sum_{j} \overline{c_{j}}=\sum_{j} \overline{b_{j}} \# \sum_{j} \overline{a_{j}}$. In particular, if $a_{j}$ and $b_{j}$ are real-valued for all $j \in \mathbb{N}$ and $\sum_{j} a_{j} \# \sum_{j} b_{j}=\sum_{j} b_{j} \# \sum_{j} a_{j}$, then $c_{j}$ are real-valued for all $j \in \mathbb{N}$. 
Proposition 3.4 [17, Proposition 4.5] For each $B \geq 0, F S_{A_{p}, \rho}^{*, \infty}\left(\mathbb{R}^{2 d} ; B\right)$ is a ring with the pointwise addition and multiplication given by \#. Moreover, the multiplication $\#: F S_{A_{p}, \rho}^{*, \infty}\left(\mathbb{R}^{2 d} ; B\right) \times F S_{A_{p}, \rho}^{*, \infty}\left(\mathbb{R}^{2 d} ; B\right) \rightarrow F S_{A_{p}, \rho}^{*, \infty}\left(\mathbb{R}^{2 d} ; B\right)$ is hypocontinuous.

The multiplicative identity of $F S_{A_{p}, \rho}^{*, \infty}\left(\mathbb{R}^{2 d} ; B\right)$ is given by $\mathbf{1}$. The \#-product of symbols corresponds to the composition of their Weyl quantisation (see the "Appendix").

\section{Hypoelliptic operators of infinite order}

This section is devoted to hypoellipticity in the context of our symbol classes. Our main goal below is to establish a semi-boundedness result. In preparation, we start by discussing $L^{2}$-realisations of the associated unbounded operators.

Lemma 4.1 $\left[17\right.$, Lemma 5.3] Let $V \subseteq \Gamma_{A_{p}, \rho}^{*, \infty}\left(\mathbb{R}^{2 d}\right)$. Assume that for every $h>0$ there exists $C>0$ (resp. there exist $h, C>0$ ) such that

$$
\left|D_{w}^{\alpha} b(w)\right| \leq C h^{|\alpha|} A_{\alpha}\langle w\rangle^{-\rho|\alpha|}, w \in \mathbb{R}^{2 d}, \alpha \in \mathbb{N}^{2 d}, b \in V .
$$

Then, for each $b \in V, b^{w}$ extends to a bounded operator on $L^{2}\left(\mathbb{R}^{d}\right)$ and the set $\left\{b^{w} \mid b \in V\right\}$ is bounded in $\mathcal{L}_{b}\left(L^{2}\left(\mathbb{R}^{d}\right), L^{2}\left(\mathbb{R}^{d}\right)\right)$. If $\left\{b_{\lambda}\right\}_{\lambda \in \Lambda} \subseteq V$ is a net that converges to $b_{0} \in V$ in the topology of $\Gamma_{A_{p}, \rho}^{*, \infty}\left(\mathbb{R}^{2 d}\right)$, then $b_{\lambda}^{w} \rightarrow b_{0}^{w}$ in $\mathcal{L}_{p}\left(L^{2}\left(\mathbb{R}^{d}\right), L^{2}\left(\mathbb{R}^{d}\right)\right)$.

Given $a \in \Gamma_{A_{p}, \rho}^{*, \infty}\left(\mathbb{R}^{2 d}\right)$, let us denote by $A$ the unbounded operator on $L^{2}\left(\mathbb{R}^{d}\right)$ with domain $\mathcal{S}^{*}\left(\mathbb{R}^{d}\right)$ defined as $A \varphi=a^{w} \varphi, \varphi \in \mathcal{S}^{*}\left(\mathbb{R}^{d}\right)$. Considering $a^{w}$ as a mapping on $\mathcal{S}^{*}\left(\mathbb{R}^{d}\right)$, its restriction to the subspace $\left\{g \in L^{2}\left(\mathbb{R}^{d}\right) \mid a^{w} g \in L^{2}\left(\mathbb{R}^{d}\right)\right\}$ defines a closed extension of $A$ which is called the maximal realisation of $A$. As standard, we denote by $\bar{A}$ the closure of $A$, also called the minimal realisation of $A$. Notice that the formal adjoint $\left(a^{w}\right)^{*}$ is in fact the pseudo-differential operator $\bar{a}^{w}$ and hence, it can be extended to a continuous operator on $\mathcal{S}^{*}\left(\mathbb{R}^{d}\right)$. One can also consider the adjoint $A^{*}$ of $A$ in $L^{2}\left(\mathbb{R}^{d}\right)$. The following result gives the precise connection between $A^{*}$ and $\left(a^{w}\right)^{*}$. Its proof is completely analogous to the one in the classical case for finite order IDOs and we omit it (see for example [13, Proposition 4.2.1, p. 160]).

Proposition 4.2 Let $a \in \Gamma_{A_{p}, \rho}^{*, \infty}\left(\mathbb{R}^{2 d}\right)$ with $A$ and $A^{*}$ defined as above. Then $A^{*}$ coincides with the maximal realisation of $\left(a^{w}\right)^{*}$, i.e. the domain of $A^{*}$ is $D\left(A^{*}\right)=$ $\left\{g \in L^{2}\left(\mathbb{R}^{d}\right) \mid\left(a^{w}\right)^{*} g \in L^{2}\left(\mathbb{R}^{d}\right)\right\}$ and $A^{*} g=\left(a^{w}\right)^{*} g, \forall g \in D\left(A^{*}\right)$.

We now introduce the notion of hypoellipticity in $\Gamma_{A_{p}, \rho}^{*, \infty}$.

Definition 4.3 $\left[4\right.$, Definition 1.1] Let $a \in \Gamma_{A_{p}, \rho}^{*, \infty}\left(\mathbb{R}^{2 d}\right)$. We say that $a$ is $\Gamma_{A_{p}, \rho^{-}}^{*, \infty}$ hypoelliptic (or, in short, simply hypoelliptic) if

(i) there exists $B>0$ such that there are $c, m>0$ (resp. for every $m>0$ there is $c>0$ ) such that 


$$
|a(x, \xi)| \geq c e^{-M(m|x|)-M(m|\xi|)}, \quad(x, \xi) \in Q_{B}^{c},
$$

(ii) there exists $B>0$ such that for every $h>0$ there is $C>0$ (resp. there are $h, C>0)$ such that

$$
\left|D_{\xi}^{\alpha} D_{x}^{\beta} a(x, \xi)\right| \leq C \frac{h^{|\alpha|+|\beta|}|a(x, \xi)| A_{\alpha} A_{\beta}}{\langle(x, \xi)\rangle^{\rho(|\alpha|+|\beta|)}}, \alpha, \beta \in \mathbb{N}^{d},(x, \xi) \in Q_{B}^{c}
$$

Operators with hypoelliptic symbols have parametrices and hence are globally regular; see the "Appendix" for the precise results.

Proposition 4.4 [17, Proposition 5.4] Let a be hypoelliptic and A be the corresponding unbounded operator on $L^{2}\left(\mathbb{R}^{d}\right)$ defined above. Then the minimal realisation $\bar{A}$ coincides with the maximal realisation. Moreover, $\bar{A}$ coincides with the restriction of $a^{w}$ on the domain of $\bar{A}$. If additionally a is real-valued, then $\bar{A}$ is a self-adjoint operator on $L^{2}\left(\mathbb{R}^{d}\right)$.

\subsection{Semi-boundedness and the spectrum of operators with positive hypoelliptic Weyl symbols}

Before we can say anything meaningful about the spectrum of operators with hypoelliptic positive Weyl symbols, we need to prove that such operators are always semi-bounded. This is a well know fact for finite order symbols. We prove here that it remains true even in the infinite order case. In order to appreciate more this result, the reader should keep in mind the operators can be of truly infinite order, i.e. the symbols are allowed to have ultrapolynomial growth; such operators then go beyond the classical Weyl-Hörmander calculus.

Proposition 4.5 Let $b \in \Gamma_{A_{p}, \rho}^{*, \infty}\left(\mathbb{R}^{2 d}\right)$ be positive hypoelliptic symbol. Then, there exists $C>0$ such that $\left(b^{w} \varphi, \varphi\right) \geq-C\|\varphi\|_{L^{2}\left(\mathbb{R}^{d}\right)}^{2}, \forall \varphi \in \mathcal{S}^{*}\left(\mathbb{R}^{d}\right)$.

Proof The proof heavily relies on the connection between the Weyl and the anti-Wick quantisation of symbols from $\Gamma_{A_{p}, \rho}^{*, \infty}\left(\mathbb{R}^{2 d}\right)$ (see [16]). For $a \in \Gamma_{A_{p}, \rho}^{*, \infty}\left(\mathbb{R}^{2 d}\right)$, we denote by $A_{a}$ its anti-Wick quantisation. By [16, Theorem 3.2], there exists $a \in \Gamma_{A_{p}, \rho}^{*, \infty}\left(\mathbb{R}^{2 d}\right)$ and a $*$-regularising operator $T$ such that $b^{w}=A_{a}+T$. By a careful inspection of the proof of the quoted result, one can find the explicit construction of $a$; it is given as follows. Start with $p_{k, j}^{\prime} \in C^{\infty}\left(\mathbb{R}^{2 d}\right), k, j \in \mathbb{N}$, defined by $p_{0,0}^{\prime}=b, p_{k, 0}^{\prime}=0$ for all $k \in \mathbb{Z}_{+}, p_{k, j}^{\prime}=0$ for all $0 \leq k<j$, and

$$
\begin{aligned}
p_{k, j}^{\prime}(x, \xi)= & \sum_{\substack{l_{1}+\cdots+l_{j}=k \\
l_{1} \geq 1, \ldots, l_{j} \geq 1}} \sum_{\left|\alpha^{(1)}+\beta^{(1)}\right|=2 l_{1}, \ldots,\left|\alpha^{(j)}+\beta^{(j)}\right|=2 l_{j}} \frac{c_{\alpha^{(1)}, \beta^{(1)} \cdots \cdots c_{\alpha^{(j)}, \beta^{(j)}}}}{\alpha^{(1) ! \beta^{(1)} ! \cdots \cdots \alpha^{(j) ! \beta^{(j) !}}}} \\
& \cdot \partial_{\xi}^{\alpha^{(1)}+\cdots+\alpha^{(j)}} \partial_{x}^{\beta^{(1)}+\cdots+\beta^{(j)}} b(x, \xi),
\end{aligned}
$$


for all $x, \xi \in \mathbb{R}^{d}, k \geq j$, where $c_{\alpha, \beta}=\pi^{-d} \int_{\mathbb{R}^{2 d}} \eta^{\alpha} y^{\beta} e^{-|y|^{2}-|\eta|^{2}} d y d \eta, \alpha, \beta \in \mathbb{N}^{d}$. Since $b$ is positive and hypoelliptic, the estimate (4.3) holds on the whole $\mathbb{R}^{2 d}$ for $b$. Repeating the proof of [16, Theorem 3.2] verbatim and using (4.3) for $b$ (which, as we mentioned, is valid on $\mathbb{R}^{2 d}$ ), we obtain the following estimate: for every $h>0$ there exists $C>0$ (resp. there exist $h, C>0$ ) such that

$$
\left|D_{w}^{\gamma} p_{k, j}^{\prime}(w)\right| \leq C h^{|\gamma|+2 k} A_{|\gamma|+2 k} b(w)\langle w\rangle^{-\rho(|\gamma|+2 k)},
$$

for all $w \in \mathbb{R}^{2 d}, \gamma \in \mathbb{N}^{2 d}, k, j \in \mathbb{N}$ (recall that $p_{k, j}^{\prime}=0$, for $0 \leq k<j, p_{k, 0}^{\prime}=0$ for $k \in \mathbb{Z}_{+}$, and $\left.p_{0,0}^{\prime}=b\right)$. Now, $a \sim \sum_{j}(-1)^{j} b_{j}$ with $b_{j}=R\left(\sum_{k} p_{k, j}^{\prime}\right)$, where $R \geq 1$ can be chosen to be the same for all $j \in \mathbb{N}$ and the following estimate holds: for every $h>0$ there exists $C>0$ (resp. there exist $h, C>0$ ) such that

$$
\left|D_{w}^{\gamma} b_{j}(w)\right| \leq C h^{|\gamma|+2 j} A_{|\gamma|+2 j} b(w)\langle w\rangle^{-\rho(|\gamma|+2 j)},
$$

for all $w \in \mathbb{R}^{2 d}, \gamma \in \mathbb{N}^{2 d}, j \in \mathbb{N}$ (cf. [16, Lemma 3.1] and its proof). Clearly $b_{0}=p_{0,0}^{\prime}=b$. In the $\left(M_{p}\right)$ case, fix $0<h^{\prime}<1$ and let $C^{\prime}>1$ be the constant for which (4.4) holds and in the $\left\{M_{p}\right\}$ case, let $h^{\prime}, C^{\prime}>1$ be the constants for which this estimate holds. If we take large enough $R^{\prime}$ such that $R^{\prime \rho} \geq 4 c_{0}^{2} H L C^{\prime}$ in the $\left(M_{p}\right)$ case and $R^{\prime \rho} \geq 4 c_{0}^{2} h^{\prime} H L C^{\prime}$ in the $\left\{M_{p}\right\}$ case respectively, then $a^{\prime}=R^{\prime}\left(\sum_{j}(-1)^{j} b_{j}\right) \in$ $\Gamma_{A_{p}, \rho}^{*, \infty}\left(\mathbb{R}^{2 d}\right)$ is real-valued and $a^{\prime} \sim a$, i.e. $a-a^{\prime} \in \mathcal{S}^{*}\left(\mathbb{R}^{2 d}\right)$ (cf. Propositions 3.1 and 3.2). Moreover, since $1-\chi_{j, R^{\prime}}=0$ on $Q_{R^{\prime} m_{j}}$ and $m_{j}^{2 j} \geq M_{2 j} /\left(c_{0} H^{2 j}\right), \forall j \in \mathbb{Z}_{+}$,

$$
\begin{aligned}
\sum_{j=1}^{\infty}\left(1-\chi_{j, R^{\prime}}(w)\right)\left|b_{j}(w)\right| & \leq C^{\prime} b(w) \sum_{j=1}^{\infty}\left(1-\chi_{j, R^{\prime}}(w)\right) h^{\prime 2 j} A_{2 j}\langle w\rangle^{-2 j \rho} \\
& \leq C^{\prime} b(w) \sum_{j=1}^{\infty} h^{\prime 2 j} A_{2 j} R^{\prime-2 j \rho} m_{j}^{-2 j \rho} \\
& \leq c_{0}^{2} C^{\prime} b(w) \sum_{j=1}^{\infty}\left(h^{\prime} H L / R^{\prime \rho}\right)^{2 j} \leq b(w) / 3
\end{aligned}
$$

Thus

$$
a^{\prime}(w)=b(w)+\sum_{j=1}^{\infty}(-1)^{j}\left(1-\chi_{j, R^{\prime}}(w)\right) b_{j}(w) \geq 2 b(w) / 3>0, \quad \forall w \in \mathbb{R}^{2 d} .
$$

Hence $\left(A_{a^{\prime}} \varphi, \varphi\right) \geq 0, \varphi \in \mathcal{S}^{*}\left(\mathbb{R}^{d}\right)$ (cf. [16, Proposition 3.4]). Observe that $A_{a^{\prime}}=$ $b^{w}+T^{\prime}$, for some $*$-regularising operator $T^{\prime}$. Since $b$ is real-valued, $\left(b^{w} \varphi, \varphi\right) \in \mathbb{R}$, $\varphi \in \mathcal{S}^{*}\left(\mathbb{R}^{d}\right)$, hence the same holds for $T^{\prime}$ too. We conclude $\left(b^{w} \varphi, \varphi\right) \geq-\left(T^{\prime} \varphi, \varphi\right) \geq$ $-\left\|T^{\prime}\right\|_{\mathcal{L}_{b}\left(L^{2}\left(\mathbb{R}^{d}\right)\right)}\|\varphi\|_{L^{2}\left(\mathbb{R}^{d}\right)}^{2}$.

Using Propositions 4.4, 4.5 and Remark 8.7, we can prove the following spectral result in the same way as in the proof of [13, Theorem 4.2.9, p. 163]. 
Proposition 4.6 Let $a \in \Gamma_{A_{p}, \rho}^{*, \infty}\left(\mathbb{R}^{2 d}\right)$ be a hypoelliptic real-valued symbol such that $|a(w)| \rightarrow \infty$ as $|w| \rightarrow \infty$ and let $A$ be the unbounded operator on $L^{2}\left(\mathbb{R}^{d}\right)$ defined by $a^{w}$. Then the closure $\bar{A}$ of $A$ is a self-adjoint operator having spectrum given by $a$ sequence of real eigenvalues either diverging to $+\infty$ or to $-\infty$ according to the sign of a at infinity. The eigenvalues have finite multiplicities and the eigenfunctions belong to $\mathcal{S}^{*}\left(\mathbb{R}^{d}\right)$. Moreover, $L^{2}\left(\mathbb{R}^{d}\right)$ has an orthonormal basis consisting of eigenfunctions of $\bar{A}$.

\section{The Weyl asymptotic formula for infinite order $\Psi$ DOs. Part I: statements of the main results}

This section is dedicated to Weyl asymptotic formulae for a large class of infinite order hypoelliptic pseudo-differential operators. We state here our main results, their proofs are postponed to Sect. 7, after obtaining some auxiliary results on the spectrum of the heat parametrix of positive hypoelliptic symbols.

We consider throughout this section a real-valued hypoelliptic symbol $a \in$ $\Gamma_{A_{p}, \rho}^{*, \infty}\left(\mathbb{R}^{2 d}\right)$ such that $a(w) \rightarrow \infty$ as $|w| \rightarrow \infty$. If we denote as $\bar{A}$ the closure of the unbounded operator on $L^{2}\left(\mathbb{R}^{d}\right)$ induced by its Weyl quantisation $a^{w}$ then we can apply Proposition 4.6 to obtain that the spectrum of the self-adjoint operator $\bar{A}$ is given by a sequence of real eigenvalues with finite multiplicities $\left\{\lambda_{j}\right\}_{j \in \mathbb{N}}$ which tends to $\infty$, where multiplicities are taken into account and the sequence is arranged in nondecreasing order $\lambda_{0} \leq \lambda_{1} \leq \lambda_{2} \leq \cdots \leq \lambda_{j} \leq \cdots$. We denote the spectral counting function of the operator $A=a^{w}$ as

$$
N(\lambda)=\sum_{\lambda_{j} \leq \lambda} 1=\#\left\{j \in \mathbb{N} \mid \lambda_{j} \leq \lambda\right\} .
$$

Our goal is to show later the following three theorems on spectral asymptotics. For these results, we will suppose that the symbol $a$ satisfies certain asymptotic bounds with respect to a comparison function $f$, which we assume throughout the rest of this section to be positive, strictly increasing, of ultrapolynomial growth of class $*$ on some interval $[Y, \infty)$, for some $Y>0$, and absolutely continuous on each compact subinterval of $[Y, \infty)$. Furthermore, we employ the notation

$$
\sigma(\lambda)=\left(f^{-1}(\lambda)\right)^{2 d} \quad \text { for large } \lambda .
$$

Theorem 5.1 Let $a \in \Gamma_{A_{p}, \rho}^{*, \infty}\left(\mathbb{R}^{2 d}\right)$ hypoelliptic, let $f$ satisfy

$$
\lim _{y \rightarrow \infty} \frac{y f^{\prime}(y)}{f(y)}=\infty,
$$

and let $\Phi$ be a positive continuous function on the sphere $\mathbb{S}^{2 d-1}$. Suppose that for each $\varepsilon \in(0,1)$ there are positive constants $c_{\epsilon}, C_{\epsilon}, B_{\epsilon}>0$ such that

$$
c_{\varepsilon} f((1-\varepsilon) r \Phi(\vartheta)) \leq a(r \vartheta) \leq C_{\varepsilon} f((1+\varepsilon) r \Phi(\vartheta)),
$$


for all $r \geq B_{\varepsilon}$ and $\vartheta \in \mathbb{S}^{2 d-1}$. Then,

$$
\begin{aligned}
\lim _{\lambda \rightarrow \infty} \frac{N(\lambda)}{\sigma(\lambda)} & =\frac{\pi}{(2 \pi)^{d+1} d} \int_{\mathbb{S}^{2 d-1}} \frac{d \vartheta}{(\Phi(\vartheta))^{2 d}}, \\
\lambda_{j} & =f\left(\gamma j^{\frac{1}{2 d}}(1+o(1))\right), \quad j \rightarrow \infty,
\end{aligned}
$$

with $\gamma=\sqrt{2 \pi} \cdot\left(2 d / \int_{\mathbb{S}^{2 d-1}}(\Phi(\vartheta))^{-2 d} d \vartheta\right)^{\frac{1}{2 d}}$, and, for each $h^{\prime}<\gamma<h$,

$$
\lim _{j \rightarrow \infty} \frac{\lambda_{j}}{f\left(h^{\prime} j^{\frac{1}{2 d}}\right)}=\infty \text { and } \lim _{j \rightarrow \infty} \frac{\lambda_{j}}{f\left(h j^{\frac{1}{2 d}}\right)}=0
$$

Note that Theorem 5.1 deals with operators which are truly of infinite order because integration of (5.2) gives that $\langle w\rangle^{\beta}=o(a(w))$ for any $\beta>0$.

The next theorem gives the Weyl asymptotic formula for a wider class of finite order pseudo-differential operators than the one that is usually discussed in the literature, see e.g. [13, Sect. 4.6]; in particular, our result is more general than [13, Theorem 4.6.1, p. 196] (see Example 5.8 below). The reader should also compare this with [20, Theorem 30.1, p. 224]; we work with different assumptions than in the quoted result and, on the other hand, we give a more explicit result concerning the asymptotic behaviour of $N(\lambda)$.

Theorem 5.2 Let $a \in \Gamma_{\rho}^{m}\left(\mathbb{R}^{2 d}\right)$ be hypoelliptic (in the $\Gamma_{\rho}^{m}$-sense). Suppose that

$$
\lim _{y \rightarrow \infty} \frac{y f^{\prime}(y)}{f(y)}=\beta \in(0, \infty)
$$

exists. If

$$
\lim _{r \rightarrow \infty} \frac{a(r \vartheta)}{f(r)}=\Phi(\vartheta)>0
$$

exists uniformly on $\vartheta \in \mathbb{S}^{2 d-1}$, then

$$
\lim _{\lambda \rightarrow \infty} \frac{N(\lambda)}{\sigma(\lambda)}=\frac{\pi}{(2 \pi)^{d+1} d} \int_{\mathbb{S}^{2 d-1}} \frac{d \vartheta}{(\Phi(\vartheta))^{2 d / \beta}}
$$

and

$$
\lambda_{j} \sim\left(\frac{\pi}{(2 \pi)^{d+1} d} \int_{\mathbb{S}^{2 d-1}} \frac{d \vartheta}{(\Phi(\vartheta))^{2 d / \beta}}\right)^{-\frac{\beta}{2 d}} f\left(j^{\frac{1}{2 d}}\right), \quad j \rightarrow \infty
$$

We will derive the following "geometric" version of Theorems 5.1 and 5.2 where the asymptotic behaviour of $N$ is given in terms of the symbol. 
Corollary 5.3 Suppose that the symbol a satisfies either the assumptions of Theorem 5.1 or those of Theorem 5.2. Then,

$$
N(\lambda) \sim \frac{1}{(2 \pi)^{d}} \int_{a(w)<\lambda} d w, \quad \lambda \rightarrow \infty
$$

If one is only interested in upper $O$-estimates on $N$, the next theorem gives such bounds under much weaker assumptions on the symbol.

Theorem 5.4 Let $a \in \Gamma_{A_{p}, \rho}^{*, \infty}\left(\mathbb{R}^{2 d}\right)$ be hypoelliptic such that

$$
C f(|w|) \leq a(w) \text { for all }|w| \geq B,
$$

for some $C, B>0$. If $f$ satisfies

$$
0<\beta^{\prime}=\liminf _{y \rightarrow \infty} \frac{y f^{\prime}(y)}{f(y)},
$$

then,

$$
\limsup _{\lambda \rightarrow \infty} \frac{N(\lambda)}{\sigma(\lambda)} \leq \frac{e}{2^{d} d !}\left(1+\frac{\Gamma\left(1+2 d / \beta^{\prime}\right)}{C^{2 d / \beta^{\prime}}}\right)
$$

and for each $0<h<\sqrt{2} C^{1 / \beta^{\prime}} e^{-1 /(2 d)} d !^{1 /(2 d)}\left(C^{2 d / \beta^{\prime}}+\Gamma\left(1+2 d / \beta^{\prime}\right)\right)^{-1 /(2 d)}$

$$
\lambda_{j} \geq f\left(h j^{\frac{1}{2 d}}\right), \quad j \geq j_{h} .
$$

Furthermore, if $f$ satisfies

$$
\lim _{y \rightarrow \infty} \frac{y f^{\prime}(y)}{f(y)}=\beta^{\prime} \in(0, \infty]
$$

then,

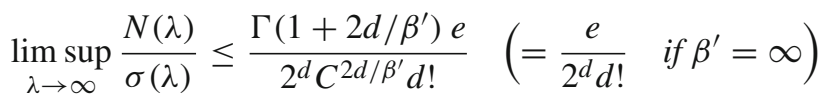

and the bound (5.15) holds for each $0<h<\sqrt{2} C^{1 / \beta^{\prime}} d !^{1 /(2 d)}\left(e \Gamma\left(1+2 d / \beta^{\prime}\right)\right)^{-1 /(2 d)}$ $\left(=\sqrt{2}(d ! / e)^{1 /(2 d)}\right.$ if $\left.\beta^{\prime}=\infty\right)$.

Remark 5.5 If $\limsup _{y \rightarrow \infty} y f^{\prime}(y) / f(y)<\infty$, Theorem 5.4 is also valid for $a \in$ $\Gamma_{\rho}^{m}\left(\mathbb{R}^{2 d}\right)$ that is $\Gamma_{\rho}^{m}$-hypoelliptic and satisfies (5.12), as the proof given in Section 7 shows. Here we get that $\lambda_{j}$ is bounded from below by a constant multiple of $f\left(j^{\frac{1}{2 d}}\right)$ for $\lambda_{j}>0$. In particular, this case applies to $f(y)=y^{\beta^{\prime}}$, where we obtain $N(\lambda)=$ $O\left(\lambda^{2 d / \beta^{\prime}}\right)$ and $\lambda_{j} \geq h^{\beta^{\prime}} j^{\beta^{\prime} /(2 d)}, j \geq j_{h}$, with the constants as in Theorem 5.4 (see also Example 5.8). 
The rest of this section is devoted to some illustrative examples. The asymptotic formulae from Examples 5.6 and 5.7 prove a result that one might expect: the eigenvalues of a truly infinite order operator are "very sparse".

Example 5.6 If $f(y)=e^{(h y)^{1 / s}}$ where $s>1$, then $\sigma(\lambda) \sim h^{-2 d}(\ln \lambda)^{2 d s}$ and, when $\Phi(\vartheta)=1$ Theorem 5.1 delivers

$$
N(\lambda) \sim 2^{-d} h^{-2 d} d !^{-1}(\ln \lambda)^{2 d s}, \quad \lambda \rightarrow \infty,
$$

and

$$
\lambda_{j}=\exp \left(2^{1 /(2 s)} h^{1 / s} d !^{1 /(2 d s)} j^{1 /(2 d s)}(1+o(1))\right), \quad j \rightarrow \infty,
$$

because here $\gamma=(d !)^{1 /(2 d)} \sqrt{2}$.

Let us give an example of a symbol that satisfies the assumptions in Theorem 5.1 with this $f$. Let

$$
a(w)=e^{(h\langle w\rangle)^{1 / s}}+a_{1}(w)
$$

where $s \geq 1 /(1-\rho)$ is such $e^{\langle w\rangle^{1 / s}}$ is of ultrapolynomial growth of class $*$ (i.e. $M_{p} \subset p !^{s}$ and $M_{p} \prec p !^{s}$, respectively) and $a_{1}$ is real-valued and satisfies the following estimate: for every $h^{\prime}>0$ there exists $C^{\prime}>0$ (resp. there exist $\left.h^{\prime}, C^{\prime}>0\right)$ such that

$$
\left|D_{w}^{\alpha} a_{1}(w)\right| \leq C^{\prime} h^{\prime|\alpha|} A_{\alpha} e^{(h\langle w\rangle)^{1 / s}}\langle w\rangle^{-\rho(|\alpha|+1)}, \forall w \in \mathbb{R}^{2 d}, \forall \alpha \in \mathbb{N}^{2 d}
$$

Clearly $a$ satisfies the bound

$$
C_{1} e^{(h|w|)^{1 / s}} \leq a(w) \leq C_{2} e^{(h|w|)^{1 / s}}, \text { for large }|w|
$$

Furthermore, since $\left|D_{w}^{\alpha}\langle w\rangle\right| \leq 2^{|\alpha|+1}|\alpha| !\langle w\rangle^{1-|\alpha|}$, for all $w \in \mathbb{R}^{2 d}, \alpha \in \mathbb{N}^{2 d}$, Pilipović and Prangoski [17, Remark 7.6] proves that $e^{(h\langle w\rangle)^{1 / s}} \in \Gamma_{A_{p}, \rho}^{*, \infty}\left(\mathbb{R}^{2 d}\right)$ and it is hypoelliptic. Because of (5.20) and (5.21), $a$ is also a hypoelliptic symbol in $\Gamma_{A_{p}, \rho}^{*, \infty}\left(\mathbb{R}^{2 d}\right)$. Hence, the asymptotic formulae (5.18) and (5.19) for $N(\lambda)$ and the eigenvalues hold true for $a^{w}=\left(e^{(h\langle\cdot\rangle)^{1 / s}}\right)^{w}+a_{1}^{w}$. We remark that given any $s>1$ the conditions are always met with $v / l \leq \rho \leq 1-1 / s, M_{p}=p !^{l}$, and $A_{p}=p !^{\nu}$ if we choose the parameters $l$ and $v$ such that $1<v<l<s$ and $v / l \leq 1-1 / s$.

More generally, let $f(y)=\tilde{M}(h y)$, where $\tilde{M}$ is the associated function of a sequence $M_{p} \subset \tilde{M}_{p}$ (resp. $M_{p} \prec \tilde{M}_{p}$ ), and $\tilde{M}_{p}$ satisfies (M.1). Then [8] $y f^{\prime}(y) / f(y)=\tilde{m}(h y) \rightarrow \infty$. In this case, when $\Phi(\vartheta)=1$ we obtain

$$
N(\lambda) \sim 2^{-d} h^{-2 d} d !^{-1}\left(\tilde{M}^{-1}(\ln \lambda)\right)^{2 d}, \quad \lambda \rightarrow \infty .
$$


Similarly for the upper bound from Theorem 5.4. In particular, if there exist $C, h>0$ such that $C e^{\tilde{M}(h|w|)} \leq a(w)$, for large $|w|$, one always has the $O$-bound

$$
N(\lambda)=O\left(\left(\tilde{M}^{-1}(\ln \lambda)\right)^{2 d}\right)
$$

If $M_{p} \prec \tilde{M}_{p}$ and there exists $B>0$ such that for every $h>0$ there exists $c>0$ such that $c e^{\tilde{M}(h|w|)} \leq a(w), \forall|w| \geq B$, then we have the effective estimate

$$
N(\lambda) \leq 2\left(2 h^{2}\right)^{-d}(e / d !)\left(\tilde{M}^{-1}(\ln \lambda)\right)^{2 d}
$$

for large enough $\lambda \geq \lambda_{h}$, which yields the $o$-bound

$$
N(\lambda)=o\left(\left(\tilde{M}^{-1}(\ln \lambda)\right)^{2 d}\right), \quad \lambda \rightarrow \infty
$$

Example 5.7 We present in this example another nontrivial instance of a hypoelliptic pseudo-differential operator of infinite order. Let $v, l, s$ be three positive numbers such that $1<v<l<s$ and $v / l \leq 1-1 / s$. Consider the entire function

$$
P(z)=1+\sum_{n=1}^{\infty} \frac{(h z)^{n}}{n^{s n}}, \quad z \in \mathbb{C},
$$

where $h$ is a positive constant, and the symbol

$$
a(w)=P(\langle w\rangle), \quad w \in \mathbb{R}^{2 d} .
$$

It is shown in [5, Sect. 3] that $a \in \Gamma_{A_{p}, \rho}^{*, \infty}\left(\mathbb{R}^{2 d}\right)$ is hypoelliptic, where $v / l \leq \rho \leq$ $1-1 / s, M_{p}=p !^{l}$, and $A_{p}=p !^{\nu}$. Denote as $N$ the spectral counting function of the Weyl quantisation of $a$ and let $\left\{\lambda_{j}\right\}_{j=0}^{\infty}$ be its sequence of eigenvalues. We will show that

$$
N(\lambda) \sim \frac{e^{2 d s}}{2^{d} h^{2 d} s^{2 d s} d !}(\ln \lambda)^{2 d s}
$$

and

$$
\lambda_{j}=\exp \left(e^{-1} s \cdot 2^{1 /(2 s)} h^{1 / s} d !^{1 /(2 d s)} j^{1 /(2 d s)}(1+o(1))\right), \quad j \rightarrow \infty .
$$

We start by noticing that, given any fixed $0<\varepsilon<1$, we have bounds

$$
c_{\varepsilon}^{\prime} P((1-\varepsilon)|w|) \leq a(w) \leq C_{\varepsilon}^{\prime} P((1+\varepsilon)|w|)
$$

for sufficiently large $w$. Next, observe that

$$
e^{-s} \exp \left(\frac{s y^{1 / s}}{e}\right) \leq \sup _{p \in \mathbb{Z}_{+}} \frac{y^{p}}{p^{s p}} \leq e^{s} \exp \left(\frac{s y^{1 / s}}{e}\right), \quad y \geq e^{s},
$$


because the only critical point of $g(t)=t \ln y-s t \ln t \operatorname{lies}$ at $t=e^{-1} y^{1 / s}$. Thus, given any arbitrary $0<\varepsilon<1$, we obtain the bounds

$$
e^{-s} \exp \left(\frac{s(h y)^{1 / s}}{e}\right) \leq P(y) \leq \frac{((1+\varepsilon) e)^{s}}{(\varepsilon+1)^{s}-1} \exp \left(\frac{(1+\varepsilon) s(h y)^{1 / s}}{e}\right), \quad y \geq e^{s} / h
$$

It then follows that the radial symbol $a$ satisfies (5.3) with $f(y)=\exp \left(e^{-1} s(h y)^{1 / s}\right)$ and the constant function $\Phi(\vartheta)=1$. Theorem 5.1 immediately yields (5.23) and (5.24).

Example 5.8 If $f(y)=y^{\beta} \ln ^{\alpha} y$, where $\beta>0$, we have that $y f^{\prime}(y) / f(y) \rightarrow \beta$ and $\sigma(\lambda) \sim\left(\beta^{\alpha} \lambda \ln ^{\alpha} \lambda\right)^{1 / \beta}$. Therefore, the conclusion of Theorem 5.2 reads in this case

$$
N(\lambda) \sim \frac{\left(\beta^{\alpha} \lambda\right)^{2 d / \beta} \pi}{(2 \pi)^{d+1} d \ln ^{2 d \alpha / \beta} \lambda} \int_{\mathbb{S}^{2 d-1}} \frac{d \vartheta}{(\Phi(\vartheta))^{2 d / \beta}}, \quad \lambda \rightarrow \infty
$$

and

$$
\lambda_{j} \sim(2 d)^{\frac{\beta-\alpha}{2 d}}(2 \pi)^{\frac{\beta}{2}}\left(\int_{\mathbb{S}^{2 d-1}} \frac{d \vartheta}{(\Phi(\vartheta))^{2 d / \beta}}\right)^{-\frac{\beta}{2 d}} j^{\frac{\beta}{2 d}} \ln \frac{\alpha}{2 d} j, \quad j \rightarrow \infty
$$

Likewise for the upper bound from Theorem 5.4.

\section{The spectrum of the heat parametrix}

Throughout this section we assume $a$ is a hypoelliptic real-valued symbol in $\Gamma_{A_{p}, \rho}^{*, \infty}\left(\mathbb{R}^{2 d}\right)$ such that $a(w) / \ln |w| \rightarrow \infty$ as $|w| \rightarrow \infty$. There exists $B \geq 1$ such that the hypoellipticity condition (4.3) for $a$ holds on $Q_{B}^{c}$ and $a(w)>0, \forall w \in Q_{B}^{c}$. Pick $\tilde{\chi} \in \mathcal{D}^{\left(A_{p}\right)}\left(\mathbb{R}^{2 d}\right)$ [resp. $\tilde{\chi} \in \mathcal{D}^{\left\{A_{p}\right\}}\left(\mathbb{R}^{2 d}\right)$ ] such that $0 \leq \tilde{\chi} \leq 1, \tilde{\chi}=1$ on $Q_{B_{1}}$, for $B_{1}>B$, and $\tilde{\chi}=0$ on the complement of a small neighbourhood of $\overline{Q_{B_{1}}}$. Then $b=(1-\tilde{\chi}) a+\tilde{\chi}$ is positive on the whole $\mathbb{R}^{2 d}$ and, in fact, it is a hypoelliptic symbol in $\Gamma_{A_{p}, \rho}^{*, \infty}\left(\mathbb{R}^{2 d}\right)$ for which the hypoellipticity condition (4.3) holds globally on $\mathbb{R}^{2 d}$.

\subsection{The heat parametrix of positive hypoelliptic symbols}

For the symbol $b$ constructed above, we can apply the theory given in [17, Subsection 7.2] for the construction of the heat parametrix. We have the following series of results.

There exist $u_{j}(t, w) \in C^{\infty}\left(\mathbb{R} \times \mathbb{R}^{2 d}\right), j \in \mathbb{N}$, such that $u_{0}(t, w)=e^{-t b(w)}$ and the following results hold.

Lemma 6.1 [17, Lemma 7.8] For every $h>0$ there exists $C>0$ (resp. there exist $h, C>0)$ such that 


$$
\left|D_{t}^{n} D_{w}^{\alpha} u_{j}(t, w)\right| \leq C n ! h^{|\alpha|+2 j} A_{|\alpha|+2 j}(b(w))^{n}\langle w\rangle^{-\rho(|\alpha|+2 j)} e^{-\frac{t}{4} b(w)},
$$

for all $\alpha \in \mathbb{N}^{2 d}, n \in \mathbb{N},(t, w) \in[0, \infty) \times \mathbb{R}^{2 d}$.

Notice that for each $R>0$, the function $u(t, w)=\sum_{n=0}^{\infty}\left(1-\chi_{n, R}(w)\right) u_{n}(t, w)=$ $R\left(\sum_{j} u_{j}\right)(t, w)$ is in $C^{\infty}\left(\mathbb{R} \times \mathbb{R}^{2 d}\right)$.

Lemma 6.2 [17, Lemma 7.10] There exists $R>1$ such that the $C^{\infty}$-function $u(t, w)=\sum_{n=0}^{\infty}\left(1-\chi_{n, R}(w)\right) u_{n}(t, w)=R\left(\sum_{j} u_{j}\right)(t, w)$ satisfies the following condition: for every $h>0$ there exists $C>0$ (resp. there exist $h, C>0$ ) such that

$$
\left|D_{t}^{n} D_{w}^{\alpha} u(t, w)\right| \leq C n ! h^{|\alpha|} A_{\alpha}(b(w))^{n}\langle w\rangle^{-\rho|\alpha|} e^{-\frac{t}{4} b(w)},
$$

for all $\alpha \in \mathbb{N}^{2 d}, n \in \mathbb{N},(t, w) \in[0, \infty) \times \mathbb{R}^{2 d}$ and

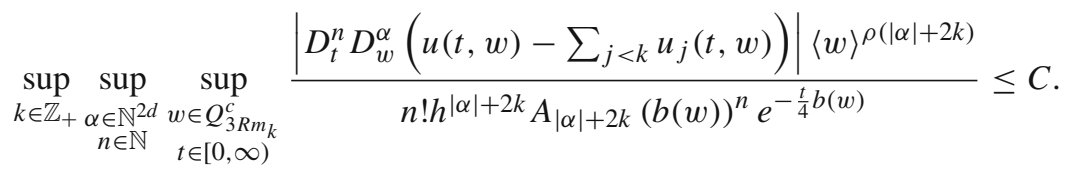

Theorem 6.3 [17, Theorem 7.11] The function $u(t, w)$ of Lemma 6.2 defines the vector-valued mapping $\mathbf{u}: t \mapsto u(t, \cdot),[0, \infty) \rightarrow \Gamma_{A_{p}, \rho}^{*, \infty}\left(\mathbb{R}^{2 d}\right)$, that belongs to $C^{\infty}\left([0, \infty) ; \Gamma_{A_{p}, \rho}^{*, \infty}\left(\mathbb{R}^{2 d}\right)\right)$. The operator-valued mapping $t \mapsto(\mathbf{u}(t))^{w}$ belongs to both $C^{\infty}\left([0, \infty) ; \mathcal{L}_{b}\left(\mathcal{S}^{*}\left(\mathbb{R}^{d}\right), \mathcal{S}^{*}\left(\mathbb{R}^{d}\right)\right)\right)$ and $C^{\infty}\left([0, \infty) ; \mathcal{L}_{b}\left(\mathcal{S}^{\prime *}\left(\mathbb{R}^{d}\right), \mathcal{S}^{\prime *}\left(\mathbb{R}^{d}\right)\right)\right)$. Moreover, $(\mathbf{u}(t))^{w}$ satisfies

$$
\left\{\begin{array}{l}
\left(\partial_{t}+b^{w}\right)(\mathbf{u}(t))^{w}=\mathbf{K}(t), t \in[0, \infty), \\
(\mathbf{u}(0))^{w}=\mathrm{Id}
\end{array}\right.
$$

where $\mathbf{K} \in C^{\infty}\left([0, \infty) ; \mathcal{L}_{b}\left(\mathcal{S}^{*}\left(\mathbb{R}^{d}\right), \mathcal{S}^{*}\left(\mathbb{R}^{d}\right)\right)\right)$.

For each $t \geq 0,(\mathbf{u}(t))^{w} \in \mathcal{L}\left(L^{2}\left(\mathbb{R}^{d}\right)\right)$ and there exists $C>0$ such that

$$
\left\|(\mathbf{u}(t))^{w}\right\|_{\mathcal{L}_{b}\left(L^{2}\left(\mathbb{R}^{d}\right)\right)} \leq C, \text { for all } t \geq 0 .
$$

The mapping $t \mapsto(\mathbf{u}(t))^{w},(0, \infty) \rightarrow \mathcal{L}_{b}\left(L^{2}\left(\mathbb{R}^{d}\right)\right)$, is continuous and $(\mathbf{u}(t))^{w} \rightarrow$ $(\mathbf{u}(0))^{w}=\mathrm{Id}$, as $t \rightarrow 0^{+}$, in $\mathcal{L}_{p}\left(L^{2}\left(\mathbb{R}^{d}\right)\right)$. Furthermore, for each $n \in \mathbb{Z}_{+}$and $t>0$, $\left(\partial_{t}^{n} \mathbf{u}(t)\right)^{w} \in \mathcal{L}\left(L^{2}\left(\mathbb{R}^{d}\right)\right)$. The mapping $t \mapsto(\mathbf{u}(t))^{w},(0, \infty) \rightarrow \mathcal{L}_{b}\left(L^{2}\left(\mathbb{R}^{d}\right)\right)$, is smooth and $\partial_{t}^{n}(\mathbf{u}(t))^{w}=\left(\partial_{t}^{n} \mathbf{u}(t)\right)^{w}$.

Since the operator $a^{w}-b^{w}=(a-b)^{w}$ is $*$-regularising (by the definition of $b$ ), (6.2) implies

$$
\left\{\begin{array}{l}
\left(\partial_{t}+a^{w}\right)(\mathbf{u}(t))^{w}=\tilde{\mathbf{K}}(t), t \in[0, \infty), \\
(\mathbf{u}(0))^{w}=\mathrm{Id},
\end{array}\right.
$$

where $\tilde{\mathbf{K}} \in C^{\infty}\left([0, \infty) ; \mathcal{L}_{b}\left(\mathcal{S}^{\prime *}\left(\mathbb{R}^{d}\right), \mathcal{S}^{*}\left(\mathbb{R}^{d}\right)\right)\right)$. 
We denote by $A$ the unbounded operator on $L^{2}\left(\mathbb{R}^{d}\right)$ defined by $a^{w}$. We apply Proposition 4.6 and obtain that the spectrum of the self-adjoint operator $\bar{A}$ is given by a sequence of real eigenvalues $\left\{\lambda_{j}\right\}_{j \in \mathbb{N}}$ which tends to $+\infty$, where the multiplicities are taken into account, and $L^{2}\left(\mathbb{R}^{d}\right)$ has an orthonormal basis $\left\{\varphi_{j}\right\}_{j \in \mathbb{N}}$ consisting of eigenfunctions of $\bar{A}$ which all belong to $\mathcal{S}^{*}\left(\mathbb{R}^{d}\right)\left(\varphi_{j}\right.$ corresponds to $\left.\lambda_{j}, j \in \mathbb{N}\right)$. For each $t \geq 0$, we define the following operator on $L^{2}\left(\mathbb{R}^{d}\right)$

$$
T(t) g=\sum_{j=0}^{\infty} e^{-t \lambda_{j}}\left(g, \varphi_{j}\right) \varphi_{j}, g \in L^{2}\left(\mathbb{R}^{d}\right)
$$

Obviously, the above series is unconditionally convergent and $T(t)$ is continuous. Furthermore, $T(t)$ is self-adjoint (one easily verifies that $(T(t) g, g) \in[0, \infty), g \in$ $L^{2}\left(\mathbb{R}^{d}\right)$, and hence it is positive) and $T(0)=$ Id. Clearly, $\{T(t)\}_{t \geq 0}$ is a $C_{0}$-semigroup.

As it will become clear later, the analysis of this semigroup is one of the key ingredients in the proofs of the main results from Sect. 5. We will show:

- $T(t)$ belongs to $\mathcal{L}\left(\mathcal{S}^{*}\left(\mathbb{R}^{d}\right), \mathcal{S}^{*}\left(\mathbb{R}^{d}\right)\right)$;

- the mapping $t \mapsto T(t),[0, \infty) \rightarrow \mathcal{L}_{b}\left(\mathcal{S}^{*}\left(\mathbb{R}^{d}\right), \mathcal{S}^{*}\left(\mathbb{R}^{d}\right)\right)$, is smooth;

- $T(t)$ and $(\mathbf{u}(t))^{w}$ are the same, modulo a smooth $*$-regularising family.

As the proofs of these facts are rather lengthy, we devote a whole subsection to them.

Remark 6.4 If $a \in \Gamma_{\rho}^{m}\left(\mathbb{R}^{2 d}\right)$ is a hypoelliptic real-valued symbol such that $a(w) \geq$ $c\langle w\rangle^{\delta}$ for some $\delta>0, \forall|w| \geq c$, one can construct its heat parametrix as well. For this purpose, one can use the same construction as in [13, Theorem 4.5.1, p. 193] (although it is there given only for elliptic symbols). In fact, defining $b \in \Gamma_{\rho}^{m}\left(\mathbb{R}^{2 d}\right)$ to be positive on $\mathbb{R}^{2 d}$ and equal to $a$ outside of a compact neighbourhood of the origin, one can repeat the proof of the quoted result verbatim to find a symbol $u(t, \cdot) \in \Gamma_{\rho}^{m}\left(\mathbb{R}^{2 d}\right), t \geq 0$, which solves (6.2) with $\mathbf{K} \in C^{\infty}\left([0, \infty) ; \mathcal{L}_{b}\left(\mathcal{S}^{\prime}\left(\mathbb{R}^{d}\right), \mathcal{S}\left(\mathbb{R}^{d}\right)\right)\right)$. Moreover, there are $u_{j}(t, w) \in C^{\infty}\left(\mathbb{R} \times \mathbb{R}^{2 d}\right), j \in \mathbb{N}$, such that

$t^{k}\left|D_{t}^{n} D_{w}^{\alpha}\left(u(t, w)-\sum_{j=0}^{J-1} u_{j}(t, w)\right)\right| \leq \frac{C_{k, n, J, t_{0}, \alpha}(b(w))^{n-k}}{\langle w\rangle^{\rho(|\alpha|+2 J)}}, w \in \mathbb{R}^{2 d}, t \in\left[0, t_{0}\right]$

$\left(t_{0}>0\right.$ can be arbitrarily chosen), where $u_{0}(t, w)=e^{-t b(w)}$ and $u_{j}$ is given as $u_{j}(t, w)=e^{-t b(w)} \sum_{l=1}^{2 j} t^{l} u_{l, j}(w), j \in \mathbb{Z}_{+}$, with symbols $u_{l, j}$ that satisfy the estimates

$$
\left|D_{w}^{\alpha} u_{l, j}(w)\right| \leq C_{l, j, \alpha}(b(w))^{l}\langle w\rangle^{-\rho(|\alpha|+2 j)}, w \in \mathbb{R}^{2 d}
$$

Notice then that $(\mathbf{u}(t))^{w}=(u(t, \cdot))^{w}$ satisfies the Eq. (6.3) for some vector-valued function $\tilde{\mathbf{K}} \in C^{\infty}\left([0, \infty) ; \mathcal{L}_{b}\left(\mathcal{S}^{\prime}\left(\mathbb{R}^{d}\right), \mathcal{S}\left(\mathbb{R}^{d}\right)\right)\right)$. 


\subsection{The analysis of the semigroup $T(t), t \geq 0$}

Lemma 6.5 The infinitesimal generator of $\{T(t)\}_{t \geq 0}$ is $-\bar{A}$.

Proof For the moment, denote as $B$ the infinitesimal generator of $\{T(t)\}_{t \geq 0}$. Fix $\psi \in \mathcal{S}^{*}\left(\mathbb{R}^{d}\right)$. Since $\bar{A} \psi=\sum_{j=0}^{\infty}\left(\bar{A} \psi, \varphi_{j}\right) \varphi_{j}$, we have $\sum_{j=0}^{\infty}\left|\left(\bar{A} \psi, \varphi_{j}\right)\right|^{2}<\infty$ and, as $\bar{A}$ is self-adjoint, we conclude

$$
\sum_{j=0}^{\infty} \lambda_{j}^{2}\left|\left(\psi, \varphi_{j}\right)\right|^{2}<\infty \text { and } \bar{A} \psi=\sum_{j=0}^{\infty} \lambda_{j}\left(\psi, \varphi_{j}\right) \varphi_{j},
$$

where the last series is unconditionally convergent in $L^{2}\left(\mathbb{R}^{d}\right)$. We have

$$
\frac{T(t) \psi-\psi}{t}+\bar{A} \psi=\sum_{j=0}^{\infty}\left(\frac{e^{-t \lambda_{j}}-1}{t}+\lambda_{j}\right)\left(\psi, \varphi_{j}\right) \varphi_{j} .
$$

Let $c>0$ be such that $\lambda_{j}>-c, j \in \mathbb{N}$. By Taylor formula, there exists $C>0$ such that $\left|e^{-t s}-1\right| \leq C t|s|$, for all $t \in[0,1], s \geq-c$. Hence $\left|e^{-t \lambda_{j}}-1\right| \leq C t\left|\lambda_{j}\right|$, for all $t \in[0,1], j \in \mathbb{N}$. Thus, letting $t \rightarrow 0^{+}$in (6.5), dominated convergence implies $t^{-1}(T(t) \psi-\psi) \rightarrow-\bar{A} \psi$ in $L^{2}\left(\mathbb{R}^{d}\right)$. Thus $-A \subset B$ and hence $-\bar{A} \subset B$ ( $B$ is closed as a generator of a $C_{0}$-semigroup). Now, for $f, g \in D(B)$, we have

$$
(B f, g)=\lim _{t \rightarrow 0^{+}}\left(t^{-1}((T(t) f-f), g)\right)=\lim _{t \rightarrow 0^{+}}\left(f, t^{-1}(T(t) g-g)\right)=(f, B g),
$$

i.e. $B \subset B^{*}$. Since $B^{*} \subset-\bar{A}^{*}=-\bar{A}$ (which follows from $-\bar{A} \subset B$ ), we conclude $-\bar{A}=B$.

Let $c>0$ be large enough such that $\lambda_{j}>-c+1, j \in \mathbb{N}$, and $\tilde{a}(w)=a(w)+c>0$, $w \in \mathbb{R}^{2 d}$. Then $\tilde{a} \in \Gamma_{A_{p}, \rho}^{*, \infty}\left(\mathbb{R}^{2 d}\right)$ is hypoelliptic and we denote by $\tilde{A}$ the corresponding unbounded operator on $L^{2}\left(\mathbb{R}^{d}\right)$. Notice that $\sigma(\overline{\tilde{A}}) \subseteq\{\lambda \in \mathbb{R} \mid \lambda>1\}$ and $\overline{\tilde{A}}$ is selfadjoint (see Proposition 4.4).

Denote by $\mathbf{P}$ the following closed sector: $\{z \in \mathbb{C} \backslash\{0\} \mid-3 \pi / 4 \leq \arg z \leq 3 \pi / 4\} \cup$ $\{0\}$. One easily verifies that there exists $\tilde{C}>0$ such that

$$
\tilde{a}(w) \leq \tilde{C}|\tilde{a}(w)+z| \text { and }|z| \leq \tilde{C}|\tilde{a}(w)+z|, \quad \forall w \in \mathbb{R}^{2 d}, \forall z \in \mathbf{P} .
$$

Of course, $\tilde{a}(w)+z \neq 0$, for all $w \in \mathbb{R}^{2 d}, z \in \mathbf{P}$. We denote by $\tilde{a}_{z}$ the symbol $\tilde{a}+z \in \Gamma_{A_{p}, \rho}^{*, \infty}\left(\mathbb{R}^{2 d}\right)$. These inequalities yield that $\tilde{a}_{z}, z \in \mathbf{P}$, are hypoelliptic and they satisfy the following uniform estimate: for every $h>0$ there exists $C>0$ (resp. there exist $h, C>0$ ) such that

$$
\left|D^{\alpha} \tilde{a}_{z}(w)\right| \leq C h^{|\alpha|} A_{\alpha}\left|\tilde{a}_{z}(w)\right|\langle w\rangle^{-\rho|\alpha|}, w \in \mathbb{R}^{2 d}, \alpha \in \mathbb{N}^{2 d}, z \in \mathbf{P} .
$$


Notice that (6.6) implies that there exist $c, C, m>0$ (resp. for every $m>0$ there exist $c, C>0$ ) such that

$$
c(1+|z|) e^{-M(m|\xi|)} e^{-M(m|x|)} \leq\left|\tilde{a}_{z}(x, \xi)\right| \leq C(1+|z|) e^{M(m|\xi|)} e^{M(m|x|)},
$$

for all $(x, \xi) \in \mathbb{R}^{2 d}, z \in \mathbf{P}$. In the Roumieu case, employing Lemma 2.1, this estimate yields the existence of $\left(k_{p}\right) \in \mathfrak{R}$ and $c, C>0$ such that

$$
c(1+|z|) e^{-N_{k_{p}}(|\xi|)} e^{-N_{k_{p}}(|x|)} \leq\left|\tilde{a}_{z}(x, \xi)\right| \leq C(1+|z|) e^{N_{k_{p}}(|\xi|)} e^{N_{k_{p}}(|x|)}
$$

for all $(x, \xi) \in \mathbb{R}^{2 d}, z \in \mathbf{P}$. Define $q_{0}^{(z)}(w)=1 / \tilde{a}_{z}(w), w \in \mathbb{R}^{2 d}$, and inductively

$$
\begin{aligned}
q_{j}^{(z)}(x, \xi)= & -q_{0}^{(z)}(x, \xi) \sum_{s=1}^{j} \sum_{|\alpha+\beta|=s} \frac{(-1)^{|\beta|}}{\alpha ! \beta ! 2^{s}} \\
& \partial_{\xi}^{\alpha} D_{x}^{\beta} q_{j-s}^{(z)}(x, \xi) \partial_{\xi}^{\beta} D_{x}^{\alpha} \tilde{a}_{z}(x, \xi), \quad(x, \xi) \in \mathbb{R}^{2 d} .
\end{aligned}
$$

In a completely analogous way as in [17, Subsection 6.2.1], one proves that $\sum_{j} q_{j}^{(z)} \in$ $F S_{A_{p}, \rho}^{*, \infty}\left(\mathbb{R}^{2 d} ; 0\right), \sum_{j} q_{j}^{(z)} \# \tilde{a}_{z}=\mathbf{1}=\tilde{a}_{z} \# \sum_{j} q_{j}^{(z)}$ in $F S_{A_{p}, \rho}^{*, \infty}\left(\mathbb{R}^{2 d} ; 0\right)$ and the following estimate holds: for every $h>0$ there exists $C>0$ (resp. there exist $h, C>0$ ) such that

$$
\left|D_{w}^{\alpha} q_{j}^{(z)}(w)\right| \leq C \frac{h^{|\alpha|+2 j} A_{|\alpha|+2 j}}{\left|\tilde{a}_{z}(w)\right|\langle w\rangle^{\rho(|\alpha|+2 j)}}, w \in \mathbb{R}^{2 d}, \alpha \in \mathbb{N}^{2 d}, j \in \mathbb{N}, z \in \mathbf{P} .
$$

This estimate together with (6.8) in the Beurling case and (6.9) in the Roumieu case respectively, implies the following: that

in the $\left(M_{p}\right)$ case, there exists $m>0$ such that for every $h>0$ there is $C>0$ such

$$
(1+|z|)\left|D_{w}^{\alpha} q_{j}^{(z)}(w)\right| \leq C h^{|\alpha|+2 j} A_{|\alpha|+2 j} e^{M(m|\xi|)} e^{M(m|x|)}\langle w\rangle^{-\rho(|\alpha|+2 j)},
$$

for all $w \in \mathbb{R}^{2 d}, \alpha \in \mathbb{N}^{2 d}, j \in \mathbb{N}, z \in \mathbf{P}$;

in the $\left\{M_{p}\right\}$ case, there exist $\left(k_{p}\right) \in \mathfrak{R}$ and $h, C>0$ such that

$$
(1+|z|)\left|D_{w}^{\alpha} q_{j}^{(z)}(w)\right| \leq C h^{|\alpha|+2 j} A_{|\alpha|+2 j} e^{N_{k_{p}}(|\xi|)} e^{N_{k_{p}}(|x|)}\langle w\rangle^{-\rho(|\alpha|+2 j)},
$$

for all $w \in \mathbb{R}^{2 d}, \alpha \in \mathbb{N}^{2 d}, j \in \mathbb{N}, z \in \mathbf{P}$. Thus, we have obtained

$$
\left\{\sum_{j}(1+|z|) q_{j}^{(z)} \mid z \in \mathbf{P}\right\} \precsim e^{M(m|\xi|)} e^{M(m|x|)} \text { in } F S_{A_{p}, \rho}^{\left(M_{p}\right), \infty}\left(\mathbb{R}^{2 d} ; 0\right) \text { and }
$$




$$
\left\{\sum_{j}(1+|z|) q_{j}^{(z)} \mid z \in \mathbf{P}\right\} \precsim e^{N_{k_{p}}(|\xi|)} e^{N_{k_{p}}(|x|)} \text { in } F S_{A_{p}, \rho}^{\left\{M_{p}\right\}, \infty}\left(\mathbb{R}^{2 d} ; 0\right)
$$

in the Beurling and the Roumieu case, respectively. Similarly, (6.8) and (6.7) yield $\left\{\tilde{a}_{z} /(1+|z|) \mid z \in \mathbf{P}\right\} \precsim e^{M(m|\xi|)} e^{M(m|x|)}$ in the Beurling case and (6.9) and (6.7) imply $\left\{\tilde{a}_{z} /(1+|z|) \mid z \in \mathbf{P}\right\} \precsim e^{N_{k_{p}}(|\xi|)} e^{N_{k_{p}}(|x|)}$ in the Roumieu case. Thus, Corollary 8.3 implies that there exist $R_{1}, R_{2}>0$ such that

$$
\left\{\mathrm{Op}_{1 / 2}\left(R_{1}\left(\sum_{j} q_{j}^{(z)}\right)\right) \tilde{a}_{z}^{w}-\mathrm{Id} \mid z \in \mathbf{P}\right\} \text { and }\left\{\tilde{a}_{z}^{w} \mathrm{Op}_{1 / 2}\left(R_{2}\left(\sum_{j} q_{j}^{(z)}\right)\right)-\mathrm{Id} \mid z \in \mathbf{P}\right\}
$$

are equicontinuous subsets of $\mathcal{L}\left(\mathcal{S}^{*}\left(\mathbb{R}^{d}\right), \mathcal{S}^{*}\left(\mathbb{R}^{d}\right)\right)$ (note that $R\left(\sum_{j}(1+|z|) q_{j}^{(z)}\right)=$ $(1+|z|) R\left(\sum_{j} q_{j}^{(z)}\right)$, for $\left.R>0\right)$. By taking $R=\max \left\{R_{1}, R_{2}\right\}$, we obtain the next result (taking larger $R_{1}$ or $R_{2}$ yields the same results because of Proposition 3.2).

Proposition 6.6 There exists $R>0$, which can be taken arbitrary large, such that

$$
\left\{\mathrm{Op}_{1 / 2}\left(R\left(\sum_{j} q_{j}^{(z)}\right)\right) \tilde{a}_{z}^{w}-\mathrm{Id} \mid z \in \mathbf{P}\right\} \text { and }\left\{\tilde{a}_{z}^{w} \mathrm{Op}_{1 / 2}\left(R\left(\sum_{j} q_{j}^{(z)}\right)\right)-\mathrm{Id} \mid z \in \mathbf{P}\right\}
$$

are equicontinuous subsets of $\mathcal{L}\left(\mathcal{S}^{* *}\left(\mathbb{R}^{d}\right), \mathcal{S}^{*}\left(\mathbb{R}^{d}\right)\right)$. Moreover, the estimate (6.10)

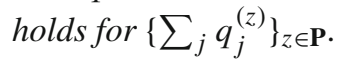

Lemma 6.7 There exists $R^{\prime}>0$ such that for all $R \geq R^{\prime}$ the following statements hold:

(i) $q_{z}:=R\left(\sum_{j} q_{j}^{(z)}\right) \in \Gamma_{A_{p}, \rho}^{*, \infty}\left(\mathbb{R}^{2 d}\right), z \in \mathbf{P}$, and for every $h>0$ there exists $C>0$ (resp. there exist $h, C>0$ ) such that

$$
\left|D_{w}^{\alpha} q_{z}(w)\right| \leq \frac{C h^{|\alpha|} A_{\alpha}}{\left|\tilde{a}_{z}(w)\right|\langle w\rangle^{\rho|\alpha|}}, w \in \mathbb{R}^{2 d}, \alpha \in \mathbb{N}^{2 d}, z \in \mathbf{P}
$$

(ii) the set $\left\{(1+|z|) q_{z}^{w} \mid z \in \mathbf{P}\right\}$ is equicontinuous in both $\mathcal{L}\left(\mathcal{S}^{*}\left(\mathbb{R}^{d}\right), \mathcal{S}^{*}\left(\mathbb{R}^{d}\right)\right)$ and $\mathcal{L}\left(\mathcal{S}^{*}\left(\mathbb{R}^{d}\right), \mathcal{S}^{*}\left(\mathbb{R}^{d}\right)\right)$.

Proof The estimate (6.10) implies $\left\{\sum_{j} q_{j}^{(z)} \mid z \in \mathbf{P}\right\} \precsim\left\{1 / \mid \tilde{a}_{z} \| z \in \mathbf{P}\right\}$ in $F S_{A_{p}, \rho}^{*, \infty}\left(\mathbb{R}^{2 d} ; 0\right)$. Thus, we can apply Proposition 3.1 to obtain the existence of $R^{\prime}>0$ such that for each $R \geq R^{\prime}, q_{z}:=R\left(\sum_{j} q_{j}^{(z)}\right) \in \Gamma_{A_{p}, \rho}^{*, \infty}\left(\mathbb{R}^{2 d}\right)$ and (6.15) is valid when $w \in Q_{B m_{1}}^{c}=Q_{B}^{c}$, for some $B=B(R)>0$. There exists $j_{0} \in \mathbb{Z}_{+}$such that $q_{z}(w)=\sum_{n=0}^{j_{0}}\left(1-\chi_{n, R}(w)\right) q_{n}^{(z)}(w)$, for all $w \in Q_{B}, z \in \mathbf{P}$. Because of (6.10) we can conclude the validity of (6.15) when $w \in Q_{B}$ as well, and the proof of $(i)$ is complete.

Fix $R \geq R^{\prime}$ and consider $q_{z}=R\left(\sum_{j} q_{j}^{(z)}\right), z \in \mathbf{P}$. As a direct consequence of (6.15) and (6.8) [resp. (6.9)], we have $\left\{(1+|z|) q_{z} \mid z \in \mathbf{P}\right\} \precsim e^{M(m|\xi|)} e^{M(m|x|)}$ (resp. $\left.\left\{(1+|z|) q_{z} \mid z \in \mathbf{P}\right\} \precsim e^{N_{k_{p}}(|\xi|)} e^{N_{k_{p}}(|x|)}\right)$. Hence, Proposition 8.1 proves (ii). 
Fix $R>0$ for which the conclusions in Proposition 6.6 and Lemma 6.7 hold and denote $q_{z}=R\left(\sum_{j} q_{j}^{(z)}\right) \in \Gamma_{A_{p}, \rho}^{*, \infty}\left(\mathbb{R}^{2 d}\right), z \in \mathbf{P}$. Since $\sigma(\overline{\tilde{A}}) \subseteq\{\lambda \in \mathbb{R} \mid \lambda>1\}$, it follows that $(z+\overline{\tilde{A}})$ is injective for each $z \in \mathbf{P}$. Hence, the operator $\tilde{a}_{z}^{w}: \mathcal{S}^{*}\left(\mathbb{R}^{d}\right) \rightarrow$ $\mathcal{S}^{*}\left(\mathbb{R}^{d}\right)$ is injective, as well. Moreover, for given $\varphi \in \mathcal{S}^{*}\left(\mathbb{R}^{d}\right)$, there exists $g \in L^{2}\left(\mathbb{R}^{d}\right)$ such that $(z+\overline{\tilde{A}}) g=\varphi($ as $z \in \rho(\overline{\tilde{A}}))$, i.e. $\tilde{a}_{z}^{w} g=\varphi$. Since $\tilde{a}_{z}$ is hypoelliptic, it is globally regular and hence $g \in \mathcal{S}^{*}\left(\mathbb{R}^{d}\right)$. Thus $\tilde{a}_{z}^{w}$ is a continuous bijection on $\mathcal{S}^{*}\left(\mathbb{R}^{d}\right)$. As $\mathcal{S}^{\left(M_{p}\right)}\left(\mathbb{R}^{d}\right)$ is an $(F)$-space and $\mathcal{S}^{\left\{M_{p}\right\}}\left(\mathbb{R}^{d}\right)$ is a $(D F S)$-space, it follows that $\mathcal{S}^{*}\left(\mathbb{R}^{d}\right)$ is a Pták space (see [19, Sect. IV. 8, p. 162]). The Pták homomorphism theorem [19, Corollary 1, p. 164] implies that $\tilde{a}_{z}^{w}$ is topological isomorphism on $\mathcal{S}^{*}\left(\mathbb{R}^{d}\right)$, for each $z \in \mathbf{P}$.

Clearly, $\left(\tilde{a}_{z}^{w}\right)^{-1}$ is the restriction of $(z+\overline{\tilde{A}})^{-1}$ to $\mathcal{S}^{*}\left(\mathbb{R}^{d}\right)$. Now, observe that

$$
\left(\tilde{a}_{z}^{w}\right)^{-1}=\left(\operatorname{Id}-q_{z}^{w} \tilde{a}_{z}^{w}\right)\left(\tilde{a}_{z}^{w}\right)^{-1}\left(\operatorname{Id}-\tilde{a}_{z}^{w} q_{z}^{w}\right)+\left(\operatorname{Id}-q_{z}^{w} \tilde{a}_{z}^{w}\right) q_{z}^{w}+q_{z}^{w},
$$

as operators on $\mathcal{S}^{*}\left(\mathbb{R}^{d}\right)$. Proposition 6.6 together with Lemma 6.7 (ii) yields that the set $\left\{(1+|z|)\left(\mathrm{Id}-q_{z}^{w} \tilde{a}_{z}^{w}\right) q_{z}^{w} \mid z \in \mathbf{P}\right\}$ is equicontinuous $*$-regularising. Proposition 6.6 implies that for each $z \in \mathbf{P}$, the operator $\left(\operatorname{Id}-q_{z}^{w} \tilde{a}_{z}^{w}\right)\left(\tilde{a}_{z}^{w}\right)^{-1}\left(\operatorname{Id}-\tilde{a}_{z}^{w} q_{z}^{w}\right)$ extends to a $*$-regularising operator. Thus, for each $z \in \mathbf{P},\left(\tilde{a}_{z}^{w}\right)^{-1}$ extends to a continuous operator on $\mathcal{S}^{\prime *}\left(\mathbb{R}^{d}\right)$. Since $\sigma(\overline{\tilde{A}}) \subseteq\{\lambda \in \mathbb{R} \mid \lambda>1\}$ and $\overline{\tilde{A}}$ is self-adjoint, Carracedo and Alix [7, Theorem 1.3 .5 , p. 21$]$ yields that $\overline{\tilde{A}}$ is sectorial with spectral angle 0 , and this in turn yields that for each $0<\delta \leq 1$ there exists $C_{\delta}>0$ such that

$$
\left\|(z+\overline{\tilde{A}})^{-1}\right\| \leq C_{\delta} /|z|
$$

for all $z \in\{\zeta \in \mathbb{C} \backslash\{0\} \mid-\pi+\delta \leq \arg \zeta \leq \pi-\delta\}$. Denote the particular constant for which (6.16) holds true on $\mathbf{P}_{*}=\mathbf{P} \backslash\{0\}$ by $\tilde{C}$. Since $\sigma(\overline{\tilde{A}}) \subseteq\{\lambda \in \mathbb{R} \mid \lambda>$ $1\}$, we have $\left\|(z+\overline{\tilde{A}})^{-1}\right\| \leq C^{\prime}$, for all $|z| \leq 1$. Now, Proposition 6.6 yields that $\left\{|z|\left(\operatorname{Id}-q_{z}^{w} \tilde{a}_{z}^{w}\right)\left(\tilde{a}_{z}^{w}\right)^{-1}\left(\operatorname{Id}-\tilde{a}_{z}^{w} q_{z}^{w}\right) \mid z \in \mathbf{P}\right\}$ and $\left\{\left(\operatorname{Id}-q_{z}^{w} \tilde{a}_{z}^{w}\right)\left(\tilde{a}_{z}^{w}\right)^{-1}\left(\mathrm{Id}-\tilde{a}_{z}^{w} q_{z}^{w}\right) \mid z \in\right.$ $\mathbf{P}\}$ are equicontinuous $*$-regularising and thus, the same holds for $\{(1+|z|)(\mathrm{Id}-$ $\left.\left.q_{z}^{w} \tilde{a}_{z}^{w}\right)\left(\tilde{a}_{z}^{w}\right)^{-1}\left(\operatorname{Id}-\tilde{a}_{z}^{w} q_{z}^{w}\right) \mid z \in \mathbf{P}\right\}$ as well. Denoting $S_{z}=\left(\operatorname{Id}-q_{z}^{w} \tilde{a}_{z}^{w}\right)\left(\tilde{a}_{z}^{w}\right)^{-1}(\operatorname{Id}-$ $\left.\tilde{a}_{z}^{w} q_{z}^{w}\right)+\left(\operatorname{Id}-q_{z}^{w} \tilde{a}_{z}^{w}\right) q_{z}^{w}$, we have $\left(\tilde{a}_{z}^{w}\right)^{-1}=q_{z}^{w}+S_{z}$. These facts, together with Lemma 6.7 ( $i \mathrm{i})$, prove the following result.

Lemma 6.8 The operators $\left(\tilde{a}_{z}^{w}\right)^{-1}, z \in \mathbf{P}$, are continuous on $\mathcal{S}^{*}\left(\mathbb{R}^{d}\right)$ and they extend to continuous operators on $\mathcal{S}^{*}\left(\mathbb{R}^{d}\right)$. The set $\left\{(1+|z|)\left(\tilde{a}_{z}^{w}\right)^{-1} \mid z \in \mathbf{P}\right\}$ is equicontinuous in $\mathcal{L}\left(\mathcal{S}^{*}\left(\mathbb{R}^{d}\right), \mathcal{S}^{*}\left(\mathbb{R}^{d}\right)\right)$ and in $\mathcal{L}\left(\mathcal{S}^{*}\left(\mathbb{R}^{d}\right), \mathcal{S}^{*}\left(\mathbb{R}^{d}\right)\right)$. Furthermore, for each $z \in \mathbf{P}$, $\left(\tilde{a}_{z}^{w}\right)^{-1}$ is exactly the restriction of $(z+\overline{\tilde{A}})^{-1}$ to $\mathcal{S}^{*}\left(\mathbb{R}^{d}\right)$.

Consider now the uniformly bounded $C_{0}$-semigroup $\tilde{T}(t)=e^{-t c} T(t), t \geq 0$. Clearly, its infinitesimal generator is $-\overline{\tilde{A}}$. Hence, Pazy [14, Theorem 5.2 (c), p. 61] proves that $\{\tilde{T}(t)\}_{t \geq 0}$ is analytic [cf. (6.16)] and [14, Theorem 7.7, p. 30] yields

$$
\tilde{T}(t)=\frac{1}{2 \pi i} \int_{\Lambda} e^{z t}(z+\overline{\tilde{A}})^{-1} d z, t>0,
$$


where $\Lambda$ is a smooth curve in $\{\zeta \in \mathbb{C} \backslash\{0\} \mid-\pi+\delta \leq \arg \zeta \leq \pi-\delta\}$ for any $0<\delta<1$, running from $\infty e^{-i \theta}$ to $\infty e^{i \theta}$ for arbitrary but fixed $\pi / 2<\theta<\pi-\delta$ and the integral is absolutely convergent for $t>0$ in $\mathcal{L}_{b}\left(L^{2}\left(\mathbb{R}^{d}\right), L^{2}\left(\mathbb{R}^{d}\right)\right)$ [cf. (6.16)].

Proposition 6.9 For each $t \geq 0, \tilde{T}(t) \in \mathcal{L}\left(\mathcal{S}^{*}\left(\mathbb{R}^{d}\right), \mathcal{S}^{*}\left(\mathbb{R}^{d}\right)\right)$. Moreover, the mapping $t \mapsto \tilde{T}(t)$ belongs to $C^{\infty}\left([0, \infty) ; \mathcal{L}_{b}\left(\mathcal{S}^{*}\left(\mathbb{R}^{d}\right), \mathcal{S}^{*}\left(\mathbb{R}^{d}\right)\right)\right)$ and its derivatives are given by $\left(d^{k} / d t^{k}\right) \tilde{T}(t)=(-1)^{k}\left(\tilde{a}^{w}\right)^{k} \tilde{T}(t), t \geq 0, k \in \mathbb{Z}_{+}$.

Proof Because of the analyticity of $z \mapsto(z+\overline{\tilde{A}})^{-1}$, we can shift the path of integration without changing the value of the integral in (6.17) to the curve $\tilde{\Lambda}=\Lambda_{1} \cup \Lambda_{2} \cup \Lambda_{3}$, where $\Lambda_{1}=\left\{r e^{-i 3 \pi / 4} \mid 1 \leq r<\infty\right\}, \Lambda_{2}=\left\{e^{i \theta} \mid-3 \pi / 4 \leq \theta \leq 3 \pi / 4\right\}$ and $\Lambda_{3}=\left\{r e^{i 3 \pi / 4} \mid 1 \leq r<\infty\right\}$. Clearly $\tilde{\Lambda} \subseteq \mathbf{P}_{*}$. For $\varphi \in \mathcal{S}^{*}\left(\mathbb{R}^{d}\right)$, we have

$$
\begin{aligned}
\tilde{T}(t) \varphi= & \frac{1-i}{2 \pi} \int_{1 / \sqrt{2}}^{\infty} e^{-r t-i r t}\left(\tilde{a}_{-r(1+i)}^{w}\right)^{-1} \varphi d r+\frac{1}{2 \pi} \int_{-3 \pi / 4}^{3 \pi / 4} e^{t e^{i \theta}} e^{i \theta}\left(\tilde{a}_{e^{i \theta}}^{w}\right)^{-1} \varphi d \theta \\
& +\frac{1+i}{2 \pi} \int_{1 / \sqrt{2}}^{\infty} e^{-r t+i r t}\left(\tilde{a}_{-r(1-i)}^{w}\right)^{-1} \varphi d r \\
= & I_{1}(t, \varphi)+I_{2}(t, \varphi)+I_{3}(t, \varphi)
\end{aligned}
$$

with absolutely convergent integrals for $t>0$ in $L^{2}\left(\mathbb{R}^{d}\right)$ [cf. (6.16); recall $\left(\tilde{a}_{z}^{w}\right)^{-1} \varphi=$ $(z+\overline{\tilde{A}})^{-1} \varphi$, for $\left.z \in \mathbf{P}, \varphi \in \mathcal{S}^{*}\left(\mathbb{R}^{d}\right)\right]$. By the properties of the Bochner integral, for each $g \in L^{2}\left(\mathbb{R}^{d}\right)$, we have

$$
\left\langle g, I_{1}(t, \varphi)\right\rangle=\frac{1-i}{2 \pi} \int_{1 / \sqrt{2}}^{\infty} e^{-r t-i r t}\left\langle g,\left(\tilde{a}_{-r(1+i)}^{w}\right)^{-1} \varphi\right\rangle d r .
$$

Our immediate goal is to prove $I_{1}(t, \varphi) \in \mathcal{S}^{*}\left(\mathbb{R}^{d}\right)$ for each $t>0$ and $\varphi \in \mathcal{S}^{*}\left(\mathbb{R}^{d}\right)$. Thus, fix $t>0$ and denote

$$
C_{t}=\int_{1 / \sqrt{2}}^{\infty} e^{-r t} d r>0
$$

Let $\varphi \in \mathcal{S}^{*}\left(\mathbb{R}^{d}\right)$ and $\varepsilon>0$ be arbitrary but fixed. By Lemma 6.8 , the set $\tilde{H}=$ $\left\{(1+|z|)\left(\tilde{a}_{z}^{w}\right)^{-1} \mid z \in \mathbf{P}\right\}$ is equicontinuous in $\mathcal{L}\left(\mathcal{S}^{*}\left(\mathbb{R}^{d}\right), \mathcal{S}^{*}\left(\mathbb{R}^{d}\right)\right)$ and hence $B=$ $\left\{(1+r \sqrt{2})\left(\tilde{a}_{-r(1+i)}^{w}\right)^{-1} \varphi \mid r \geq 1 / \sqrt{2}\right\}$ is bounded in $\mathcal{S}^{*}\left(\mathbb{R}^{d}\right)$. Thus, the absolute polar of $\left(C_{t} / \varepsilon\right) B$, which we denote by $W=\left(\left(C_{t} / \varepsilon\right) B\right)^{\circ}$, is a neighbourhood of zero in $\mathcal{S}^{\prime *}\left(\mathbb{R}^{d}\right)$. Hence, employing (6.18) for $g \in W \cap L^{2}\left(\mathbb{R}^{d}\right)$, we have

$$
\left|\left\langle g, I_{1}(t, \varphi)\right\rangle\right| \leq \int_{1 / \sqrt{2}}^{\infty} e^{-r t}\left|\left\langle g,\left(\tilde{a}_{-r(1+i)}^{w}\right)^{-1} \varphi\right\rangle\right| d r \leq \varepsilon .
$$

Thus, the mapping $g \mapsto\left\langle g, I_{1}(t, \varphi)\right\rangle, L^{2}\left(\mathbb{R}^{d}\right) \rightarrow \mathbb{C}$, is continuous when we equip $L^{2}\left(\mathbb{R}^{d}\right)$ with the topology induced on it by $\mathcal{S}^{*}\left(\mathbb{R}^{d}\right)$. Hence $g \mapsto\left\langle g, I_{1}(t, \varphi)\right\rangle$ can be continuously extended to a functional on $\mathcal{S}^{*}\left(\mathbb{R}^{d}\right)$, i.e. $I_{1}(t, \varphi) \in \mathcal{S}^{*}\left(\mathbb{R}^{d}\right)$. Let $g \in \mathcal{S}^{*}\left(\mathbb{R}^{d}\right)$. There exist $g_{j} \in L^{2}\left(\mathbb{R}^{d}\right), j \in \mathbb{Z}_{+}$, such that $g_{j} \rightarrow g$ in $\mathcal{S}^{\prime *}\left(\mathbb{R}^{d}\right)$ 
$\left(L^{2}\left(\mathbb{R}^{d}\right)\right.$ is sequentially dense in $\left.\mathcal{S}^{*}\left(\mathbb{R}^{d}\right)\right)$. The function $r \mapsto\left\langle g,\left(\tilde{a}_{-r(1+i)}^{w}\right)^{-1} \varphi\right\rangle$, $[1 / \sqrt{2}, \infty) \rightarrow \mathbb{C}$, is measurable since it is the pointwise limit of the sequence of continuous functions $r \mapsto\left\langle g_{j},\left(\tilde{a}_{-r(1+i)}^{w}\right)^{-1} \varphi\right\rangle,[1 / \sqrt{2}, \infty) \rightarrow \mathbb{C}$. Because of the equicontinuity of $\tilde{H}$ and the fact that $\left\{g_{j} \mid j \in \mathbb{Z}_{+}\right\}$is bounded in $\mathcal{S}^{\prime *}\left(\mathbb{R}^{d}\right)$, we can conclude the existence of $C^{\prime}>0$ such that $\left|\left\langle g_{j},\left(\tilde{a}_{-r(1+i)}^{w}\right)^{-1} \varphi\right\rangle\right| \leq C^{\prime}$, for all $r \in$ $[1 / \sqrt{2}, \infty), j \in \mathbb{Z}_{+}$. Applying the dominated convergence theorem to (6.18) with $g_{j}$ in place of $g$, we can conclude that (6.18) is valid for $g \in \mathcal{S}^{*}\left(\mathbb{R}^{d}\right)$. Next, we prove that for each $t>0$, the mapping $\varphi \mapsto I_{1}(t, \varphi), \mathcal{S}^{*}\left(\mathbb{R}^{d}\right) \rightarrow \mathcal{S}^{*}\left(\mathbb{R}^{d}\right)$, is continuous. Let $V$ be a closed convex circled neighbourhood of zero in $\mathcal{S}^{*}\left(\mathbb{R}^{d}\right)$, which, without loss of generality, we can assume to be the absolute polar $B^{\prime \circ}$ of a bounded set $B^{\prime}$ in $\mathcal{S}^{*}\left(\mathbb{R}^{d}\right)$. Since

$$
\tilde{H}^{\prime}=\left\{(1+|z|)^{t}\left(\left(\tilde{a}_{z}^{w}\right)^{-1}\right) \mid z \in \mathbf{P}\right\}
$$

is equicontinuous in $\mathcal{L}\left(\mathcal{S}^{*}\left(\mathbb{R}^{d}\right), \mathcal{S}^{*}\left(\mathbb{R}^{d}\right)\right.$ ) (cf. Lemma 6.8 and [12, Theorem 6, p. $138])$, the set $B_{1}^{\prime}=\left\{(1+|z|)^{t}\left(\left(\tilde{a}_{z}^{w}\right)^{-1}\right) g \mid z \in \mathbf{P}, g \in B^{\prime}\right\}$ is bounded in $\mathcal{S}^{*}\left(\mathbb{R}^{d}\right)$. Hence $V_{1}=\left(C_{t} B_{1}^{\prime}\right)^{\circ}$ is a neighbourhoods of zero in $\mathcal{S}^{*}\left(\mathbb{R}^{d}\right)$ [see (6.19) for the definition of $C_{t}$ ]. Employing (6.18), one easily verifies $\left|\left\langle g, I_{1}(t, \varphi)\right\rangle\right| \leq 1$, for all $\varphi \in V_{1}$ and $g \in B^{\prime}$, which proves the desired continuity. Next, we prove that the mapping $t \mapsto I_{1}(t, \cdot)$ belongs to $C\left((0, \infty) ; \mathcal{L}_{b}\left(\mathcal{S}^{*}\left(\mathbb{R}^{d}\right), \mathcal{S}^{*}\left(\mathbb{R}^{d}\right)\right)\right)$. Fix $t_{0}>0$ and let $\delta>0$ be small enough such that $\left[t_{0}-\delta, t_{0}+\delta\right] \subseteq(0, \infty)$. Consider the following subset of $\mathcal{L}\left(\mathcal{S}^{*}\left(\mathbb{R}^{d}\right), \mathcal{S}^{*}\left(\mathbb{R}^{d}\right)\right)$ :

$$
H_{1}=\left\{\varphi \mapsto I_{1}(t, \varphi) \mid t \in\left[t_{0}-\delta, t_{0}+\delta\right]\right\} .
$$

Employing (6.18) together with the fact that $\tilde{H}^{\prime}$ is equicontinuous in $\mathcal{L}\left(\mathcal{S}^{*}\left(\mathbb{R}^{d}\right)\right.$, $\mathcal{S}^{\prime *}\left(\mathbb{R}^{d}\right)$ ) (see (6.20) for the definition of $\tilde{H}^{\prime}$ ), one can easily derive that $H_{1}$ is a bounded set in $\mathcal{L}_{\sigma}\left(\mathcal{S}^{*}\left(\mathbb{R}^{d}\right), \mathcal{S}^{*}\left(\mathbb{R}^{d}\right)\right)$ and hence equicontinuous $\left(\mathcal{S}^{*}\left(\mathbb{R}^{d}\right)\right.$ is barrelled). Fix $\varphi \in \mathcal{S}^{*}\left(\mathbb{R}^{d}\right)$ and a neighbourhood of zero $V$ in $\mathcal{S}^{*}\left(\mathbb{R}^{d}\right)$ for which we may assume that it is the absolute polar $B^{\prime \circ}$ of a convex circled bounded subset $B^{\prime}$ of $\mathcal{S}^{\prime *}\left(\mathbb{R}^{d}\right)$. Let $1 \leq C<\infty$ be large enough such that $C \geq \sup \left\{\mid\left\langle g,\left(\tilde{a}_{z}^{w}\right)^{-1} \varphi\right\rangle \| z \in \mathbf{P}, g \in B^{\prime}\right\}$. Then, employing (6.18), we have

$\sup _{g \in B^{\prime}}\left|\left\langle g, I_{1}(t, \varphi)-I_{1}\left(t_{0}, \varphi\right)\right\rangle\right| \leq C \int_{1 / \sqrt{2}}^{\infty} e^{-r\left(t_{0}-\delta\right)}\left|e^{-r\left(t-t_{0}+\delta\right)} e^{-i r t}-e^{-r \delta} e^{-i r t_{0}}\right| d r$,

for all $t \in\left[t_{0}-\delta, t_{0}+\delta\right]$. The dominated convergence theorem implies that there exists $0<\varepsilon<\delta$ such that $I_{1}(t, \varphi)-I_{1}\left(t_{0}, \varphi\right) \in B^{\prime \circ}=V$, for all $t \in\left[t_{0}-\varepsilon, t_{0}+\varepsilon\right]$. Hence $I_{1}(t, \cdot) \rightarrow I_{1}\left(t_{0}, \cdot\right)$, as $t \rightarrow t_{0}$, in $\mathcal{L}_{\sigma}\left(\mathcal{S}^{*}\left(\mathbb{R}^{d}\right), \mathcal{S}^{*}\left(\mathbb{R}^{d}\right)\right)$. As $H_{1}$ is equicontinuous, the Banach-Steinhaus theorem [19, Theorem 4.5, p. 85] implies that the convergence holds in the topology of precompact convergence and, as $\mathcal{S}^{*}\left(\mathbb{R}^{d}\right)$ is Montel, it also holds in $\mathcal{L}_{b}\left(\mathcal{S}^{*}\left(\mathbb{R}^{d}\right), \mathcal{S}^{*}\left(\mathbb{R}^{d}\right)\right)$. This proves the continuity of the mapping $t \mapsto I_{1}(t, \cdot)$, $(0, \infty) \rightarrow \mathcal{L}_{b}\left(\mathcal{S}^{*}\left(\mathbb{R}^{d}\right), \mathcal{S}^{*}\left(\mathbb{R}^{d}\right)\right)$. 
In an analogous fashion one proves that for each $t>0$, the mappings $\varphi \mapsto I_{2}(t, \varphi)$ and $\varphi \mapsto I_{3}(t, \varphi)$ belong to $\mathcal{L}\left(\mathcal{S}^{*}\left(\mathbb{R}^{d}\right), \mathcal{S}^{*}\left(\mathbb{R}^{d}\right)\right)$ and the mappings $t \mapsto I_{2}(t, \cdot)$ and $t \mapsto I_{3}(t, \cdot),(0, \infty) \rightarrow \mathcal{L}_{b}\left(\mathcal{S}^{*}\left(\mathbb{R}^{d}\right), \mathcal{S}^{*}\left(\mathbb{R}^{d}\right)\right)$, are continuous.

Thus, we obtain $\tilde{T}(t) \in \mathcal{L}\left(\mathcal{S}^{*}\left(\mathbb{R}^{d}\right), \mathcal{S}^{*}\left(\mathbb{R}^{d}\right)\right)$, for each $t>0$, and also $t \mapsto \tilde{T}(t) \in$ $C\left((0, \infty) ; \mathcal{L}_{b}\left(\mathcal{S}^{*}\left(\mathbb{R}^{d}\right), \mathcal{S}^{*}\left(\mathbb{R}^{d}\right)\right)\right)$. Next, we prove the continuity at 0 . For each $t>$ 0 , we shift the path of integration in (6.17) to $\tilde{\Lambda}_{t}=\tilde{\Lambda}_{1, t} \cup \tilde{\Lambda}_{2, t} \cup \tilde{\Lambda}_{3, t}$, where $\tilde{\Lambda}_{1, t}=\left\{r e^{-i 3 \pi / 4} \mid 1 / t \leq r<\infty\right\}, \tilde{\Lambda}_{2, t}=\left\{t^{-1} e^{i \theta} \mid-3 \pi / 4 \leq \theta \leq 3 \pi / 4\right\}$ and $\tilde{\Lambda}_{3, t}=\left\{r e^{i 3 \pi / 4} \mid 1 / t \leq r<\infty\right\}$. Clearly $\tilde{\Lambda}_{t} \subseteq \mathbf{P}_{*}$. For $\varphi \in \mathcal{S}^{*}\left(\mathbb{R}^{\bar{d}}\right)$, we have

$$
\begin{aligned}
\tilde{T}(t) \varphi= & \frac{1-i}{2 \pi} \int_{(t \sqrt{2})^{-1}}^{\infty} e^{-r t-i r t}\left(\tilde{a}_{-r(1+i)}^{w}\right)^{-1} \varphi d r+\frac{1}{2 \pi t} \int_{-3 \pi / 4}^{3 \pi / 4} e^{e^{i \theta}} e^{i \theta}\left(\tilde{a}_{t^{-1}}^{w} e^{i \theta}\right)^{-1} \varphi d \theta \\
& +\frac{1+i}{2 \pi} \int_{(t \sqrt{2})^{-1}}^{\infty} e^{-r t+i r t}\left(\tilde{a}_{-r(1-i)}^{w}\right)^{-1} \varphi d r \\
= & \tilde{I}_{1}(t, \varphi)+\tilde{I}_{2}(t, \varphi)+\tilde{I}_{3}(t, \varphi) .
\end{aligned}
$$

Analogously as above, one establishes that, for each $t>0$ and $\varphi \in \mathcal{S}^{*}\left(\mathbb{R}^{d}\right)$, one has $\tilde{I}_{1}(t, \varphi), \tilde{I}_{2}(t, \varphi), \tilde{I}_{3}(t, \varphi) \in \mathcal{S}^{*}\left(\mathbb{R}^{d}\right)$. By similar techniques as in the proof of the validity of (6.18) for $g \in \mathcal{S}^{* *}\left(\mathbb{R}^{d}\right)$, one can prove that for each $g \in \mathcal{S}^{*}\left(\mathbb{R}^{d}\right)$, $\varphi \in \mathcal{S}^{*}\left(\mathbb{R}^{d}\right)$ and $t>0$ we have

$$
\begin{aligned}
\left\langle g, \tilde{I}_{1}(t, \varphi)\right\rangle & =\frac{1-i}{2 \pi} \int_{(t \sqrt{2})^{-1}}^{\infty} e^{-r t-i r t}\left\langle g,\left(\tilde{a}_{-r(1+i)}^{w}\right)^{-1} \varphi\right\rangle d r, \\
\left\langle g, \tilde{I}_{2}(t, \varphi)\right\rangle & =\frac{1}{2 \pi t} \int_{-3 \pi / 4}^{3 \pi / 4} e^{e^{i \theta}} e^{i \theta}\left\langle g,\left(\tilde{a}_{t^{-1}}^{w} e^{i \theta}\right)^{-1} \varphi\right\rangle d \theta, \\
\left\langle g, \tilde{I}_{3}(t, \varphi)\right\rangle & =\frac{1+i}{2 \pi} \int_{(t \sqrt{2})^{-1}}^{\infty} e^{-r t+i r t}\left\langle g,\left(\tilde{a}_{-r(1-i)}^{w}\right)^{-1} \varphi\right\rangle d r .
\end{aligned}
$$

Fix $\varphi \in \mathcal{S}^{*}\left(\mathbb{R}^{d}\right)$ and a bounded subset $B^{\prime}$ of $\mathcal{S}^{\prime *}\left(\mathbb{R}^{d}\right)$. The equicontinuity of $\tilde{H}^{\prime}$ [cf. (6.20)] implies the existence of $C^{\prime}>0$ such that $\left|\left\langle g,\left(\tilde{a}_{-r(1+i)}^{w}\right)^{-1} \varphi\right\rangle\right| \leq C^{\prime} /(1+r \sqrt{2})$, for all $g \in B^{\prime}, r \in[0, \infty)$, and hence, a change of variables yields

$$
\left|\left\langle g, \tilde{I}_{1}(t, \varphi)\right\rangle\right| \leq \frac{C^{\prime}}{\pi} \int_{1 / \sqrt{2}}^{\infty} \frac{e^{-s}}{t+s \sqrt{2}} d s \leq C^{\prime} \int_{1 / \sqrt{2}}^{\infty} e^{-s} d s \leq C^{\prime},
$$

for all $g \in B^{\prime}, t>0$. Similarly, there exists $C^{\prime \prime}>0$ such that $\left|\left\langle g, \tilde{I}_{3}(t, \varphi)\right\rangle\right| \leq C^{\prime \prime}$, for all $g \in B^{\prime}, t>0$. Again, the equicontinuity of $\tilde{H}^{\prime}$ yields the existence of $C^{\prime \prime \prime}>0$ such that $\left|\left\langle g,\left(\tilde{a}_{t^{-1} e^{i \theta}}^{w}\right)^{-1} \varphi\right\rangle\right| \leq C^{\prime \prime \prime} /\left(1+t^{-1}\right)$, for all $g \in B^{\prime}, \theta \in[-3 \pi / 4,3 \pi / 4]$, $t>0$. Hence

$$
\left|\left\langle g, \tilde{I}_{2}(t, \varphi)\right\rangle\right| \leq \frac{C^{\prime \prime \prime}}{2 \pi t} \int_{-3 \pi / 4}^{3 \pi / 4} \frac{e^{\cos \theta}}{1+t^{-1}} d \theta \leq 3 e C^{\prime \prime \prime} / 4,
$$

for all $g \in B^{\prime}, t>0$. We conclude that there exists $C>0$ such that $|\langle g, \tilde{T}(t) \varphi\rangle| \leq$ $C$, for all $g \in B^{\prime}, t>0$. This proves that $\{\tilde{T}(t) \mid t>0\}$ is bounded and hence 
equicontinuous in $\mathcal{L}\left(\mathcal{S}^{*}\left(\mathbb{R}^{d}\right), \mathcal{S}^{*}\left(\mathbb{R}^{d}\right)\right)$. Consequently, the same holds for $\{\tilde{T}(t) \mid t \geq$ $0\}$ ( since $\tilde{T}(0)=\mathrm{Id})$. This immediately yields the equicontinuity of $\left\{\tilde{a}^{w} \tilde{T}(t) \mid t \geq 0\right\}$ in $\mathcal{L}\left(\mathcal{S}^{*}\left(\mathbb{R}^{d}\right), \mathcal{S}^{*}\left(\mathbb{R}^{d}\right)\right)$. Since $\{\tilde{T}(t)\}_{t \geq 0}$ is a $C_{0}$-semigroup with infinitesimal generator $-\overline{\tilde{A}}$, we have

$$
\tilde{T}(t) \varphi-\varphi=-\int_{0}^{t} \overline{\tilde{A}} \tilde{T}(s) \varphi d s=-\int_{0}^{t} \tilde{a}^{w} \tilde{T}(s) \varphi d s, \forall \varphi \in \mathcal{S}^{*}\left(\mathbb{R}^{d}\right), t>0 .
$$

Employing the equicontinuity of $\left\{\tilde{a}^{w} \tilde{T}(t) \mid t \geq 0\right\}$ in $\mathcal{L}\left(\mathcal{S}^{*}\left(\mathbb{R}^{d}\right), \mathcal{S}^{*}\left(\mathbb{R}^{d}\right)\right)$ and using similar arguments as in the proof of the validity of (6.18) for $g \in \mathcal{S}^{*}\left(\mathbb{R}^{d}\right)$, one can prove that for each $g \in \mathcal{S}^{*}\left(\mathbb{R}^{d}\right), \varphi \in \mathcal{S}^{*}\left(\mathbb{R}^{d}\right)$ and $t>0$ we have

$$
\langle g, \tilde{T}(t) \varphi-\varphi\rangle=-\int_{0}^{t}\left\langle g, \tilde{a}^{w} \tilde{T}(s) \varphi\right\rangle d s .
$$

For fixed $\varphi \in \mathcal{S}^{*}\left(\mathbb{R}^{d}\right)$ and a bounded subset $B^{\prime}$ of $\mathcal{S}^{*}\left(\mathbb{R}^{d}\right)$, the equicontinuity of the set $\left\{\tilde{a}^{w} \tilde{T}(t) \mid t \geq 0\right\}$ in $\mathcal{L}\left(\mathcal{S}^{*}\left(\mathbb{R}^{d}\right), \mathcal{S}^{*}\left(\mathbb{R}^{d}\right)\right)$ proves the existence of $C>0$ such that $\left|\left\langle g, \tilde{a}^{w} \tilde{T}(t) \varphi\right\rangle\right| \leq C$, for all $g \in B^{\prime}, t \geq 0$. Thus, (6.21) yields $|\langle g, \tilde{T}(t) \varphi-\varphi\rangle| \leq C t$, for all $g \in B^{\prime}, t>0$. Hence, there exists $\varepsilon>0$ such that for all $0<t<\varepsilon$, $\tilde{T}(t) \varphi-\varphi \in B^{\prime \circ}$, which proves that $\tilde{T}(t) \rightarrow \tilde{T}(0)=\mathrm{Id}$, as $t \rightarrow 0^{+}$, in $\mathcal{L}_{\sigma}\left(\mathcal{S}^{*}\left(\mathbb{R}^{d}\right), \mathcal{S}^{*}\left(\mathbb{R}^{d}\right)\right)$. Since $\{\tilde{T}(t) \mid t \geq 0\}$ is equicontinuous in $\mathcal{L}\left(\mathcal{S}^{*}\left(\mathbb{R}^{d}\right), \mathcal{S}^{*}\left(\mathbb{R}^{d}\right)\right)$, the Banach-Steinhaus theorem [19, Theorem 4.5, p. 85] yields that the convergence holds in the topology of precompact convergence and, as $\mathcal{S}^{*}\left(\mathbb{R}^{d}\right)$ is Montel, the convergence also holds in $\mathcal{L}_{b}\left(\mathcal{S}^{*}\left(\mathbb{R}^{d}\right), \mathcal{S}^{*}\left(\mathbb{R}^{d}\right)\right)$. This proves that $t \mapsto \tilde{T}(t)$ belongs to $C\left([0, \infty) ; \mathcal{L}_{b}\left(\mathcal{S}^{*}\left(\mathbb{R}^{d}\right), \mathcal{S}^{*}\left(\mathbb{R}^{d}\right)\right)\right)$.

Observe now that for $t>t_{0} \geq 0, g \in \mathcal{S}^{*}\left(\mathbb{R}^{d}\right)$ and $\varphi \in \mathcal{S}^{*}\left(\mathbb{R}^{d}\right)$, (6.21) implies

$$
\begin{aligned}
& \frac{\left\langle g, \tilde{T}(t) \varphi-\tilde{T}\left(t_{0}\right) \varphi\right\rangle}{t-t_{0}}+\left\langle g, \tilde{a}^{w} \tilde{T}\left(t_{0}\right) \varphi\right\rangle \\
& =-\frac{1}{t-t_{0}} \int_{t_{0}}^{t}\left\langle g, \tilde{a}^{w}\left(\tilde{T}(s)-\tilde{T}\left(t_{0}\right)\right) \varphi\right\rangle d s .
\end{aligned}
$$

Let $B$ be a bounded subset of $\mathcal{S}^{*}\left(\mathbb{R}^{d}\right)$ and $V$ a neighbourhood of zero in $\mathcal{S}^{*}\left(\mathbb{R}^{d}\right)$. Consider the neighbourhood of zero $M(B, V)=\left\{S \in \mathcal{L}\left(\mathcal{S}^{*}\left(\mathbb{R}^{d}\right), \mathcal{S}^{*}\left(\mathbb{R}^{d}\right)\right) \mid S(B) \subseteq\right.$ $V\}$ in $\mathcal{L}_{b}\left(\mathcal{S}^{*}\left(\mathbb{R}^{d}\right), \mathcal{S}^{*}\left(\mathbb{R}^{d}\right)\right)$. We may of course assume $V$ is the absolute polar $B^{\prime \circ}$ of a bounded set $B^{\prime}$ in $\mathcal{S}^{* *}\left(\mathbb{R}^{d}\right)$. Then $B_{1}^{\prime}={ }^{t}\left(\tilde{a}^{w}\right) B^{\prime}$ is bounded in $\mathcal{S}^{* *}\left(\mathbb{R}^{d}\right)$ and hence its absolute polar $V_{1}=B_{1}^{\prime \circ}$ is a neighbourhood of zero in $\mathcal{S}^{*}\left(\mathbb{R}^{d}\right)$. Since $t \mapsto \tilde{T}(t) \in$ $C\left([0, \infty) ; \mathcal{L}_{b}\left(\mathcal{S}^{*}\left(\mathbb{R}^{d}\right), \mathcal{S}^{*}\left(\mathbb{R}^{d}\right)\right)\right)$, there exists $\varepsilon>0$ such that for all $t \in\left[t_{0}, t_{0}+\varepsilon\right]$, we have $\tilde{T}(t)-\tilde{T}\left(t_{0}\right) \in M\left(B, V_{1}\right)$. Thus, (6.22) implies $\left(t-t_{0}\right)^{-1}\left(\tilde{T}(t)-\tilde{T}\left(t_{0}\right)\right)+$ $\tilde{a}^{w} \tilde{T}\left(t_{0}\right) \in M(B, V)$, for all $t \in\left(t_{0}, t_{0}+\varepsilon\right]$, i.e. the right derivative of $t \mapsto \tilde{T}(t)$, $[0, \infty) \rightarrow \mathcal{L}_{b}\left(\mathcal{S}^{*}\left(\mathbb{R}^{d}\right), \mathcal{S}^{*}\left(\mathbb{R}^{d}\right)\right)$, is $-\tilde{a}^{w} \tilde{T}\left(t_{0}\right)$. Similarly, the left derivative at $t_{0}>0$ is $-\tilde{a}^{w} \tilde{T}\left(t_{0}\right)$. Hence, $t \mapsto \tilde{T}(t),[0, \infty) \rightarrow \mathcal{L}_{b}\left(\mathcal{S}^{*}\left(\mathbb{R}^{d}\right), \mathcal{S}^{*}\left(\mathbb{R}^{d}\right)\right)$, is differentiable and $(d / d t) \tilde{T}(t)=-\tilde{a}^{w} \tilde{T}(t)$. As $t \mapsto-\tilde{a}^{w} \tilde{T}(t)$ is continuous, $t \mapsto \tilde{T}(t)$ is of class $C^{1}$ and now, the equality $(d / d t) \tilde{T}(t)=-\tilde{a}^{w} \tilde{T}(t)$ readily implies that $t \mapsto \tilde{T}(t)$ is in $C^{\infty}\left([0, \infty) ; \mathcal{L}_{b}\left(\mathcal{S}^{*}\left(\mathbb{R}^{d}\right), \mathcal{S}^{*}\left(\mathbb{R}^{d}\right)\right)\right)$ and $\left(d^{k} / d t^{k}\right) \tilde{T}(t)=(-1)^{k}\left(\tilde{a}^{w}\right)^{k} \tilde{T}(t), k \in \mathbb{Z}_{+}$. 
As a direct consequence of the previous proposition we then have,

Theorem 6.10 We have $T(t) \in \mathcal{L}\left(\mathcal{S}^{*}\left(\mathbb{R}^{d}\right), \mathcal{S}^{*}\left(\mathbb{R}^{d}\right)\right)$ for each $t \geq 0$. Moreover, the mapping $t \mapsto T(t)$ belongs to $C^{\infty}\left([0, \infty) ; \mathcal{L}_{b}\left(\mathcal{S}^{*}\left(\mathbb{R}^{d}\right), \mathcal{S}^{*}\left(\mathbb{R}^{d}\right)\right)\right)$ and one has $\left(d^{k} / d t^{k}\right) T(t)=(-1)^{k}\left(a^{w}\right)^{k} T(t), t \geq 0, k \in \mathbb{Z}_{+}$.

Since $T(t)$ solves $(6.3)$ with $\tilde{\mathbf{K}}(t)=0$, we obtain

$$
(\mathbf{u}(t))^{w} \varphi-T(t) \varphi=\int_{0}^{t} T(t-s) \tilde{\mathbf{K}}(s) \varphi d s, \varphi \in \mathcal{S}^{*}\left(\mathbb{R}^{d}\right) .
$$

Theorem 6.10 then implies that for each $t>0$, the mapping $s \mapsto T(t-s) \tilde{\mathbf{K}}(s)$ belongs to $C^{\infty}\left([0, t] ; \mathcal{L}_{b}\left(\mathcal{S}^{*}\left(\mathbb{R}^{d}\right), \mathcal{S}^{*}\left(\mathbb{R}^{d}\right)\right)\right)$. For $t \geq 0$ and $f \in \mathcal{S}^{*}\left(\mathbb{R}^{d}\right)$, define

$$
\mathbf{Q}(t) f=\int_{0}^{t} T(t-s) \tilde{\mathbf{K}}(s) f d s \in L^{2}\left(\mathbb{R}^{d}\right) .
$$

Similarly as in the proof of Proposition 6.9, one verifies $\mathbf{Q}(t) f \in \mathcal{S}^{*}\left(\mathbb{R}^{d}\right)$ and, for each $g \in \mathcal{S}^{*}\left(\mathbb{R}^{d}\right)$,

$$
\langle g, \mathbf{Q}(t) f\rangle=\int_{0}^{t}\langle g, T(t-s) \tilde{\mathbf{K}}(s) f\rangle d s .
$$

Again, employing analogous techniques as in the proof of Proposition 6.9, one can prove $f \mapsto \mathbf{Q}(t) f \in \mathcal{L}\left(\mathcal{S}^{*}\left(\mathbb{R}^{d}\right), \mathcal{S}^{*}\left(\mathbb{R}^{d}\right)\right)$, for each $t \geq 0$. Using the properties of $T(t)$ and $\tilde{\mathbf{K}}(t)$, one readily checks that the mapping $(t, s) \mapsto T(t-s) \tilde{\mathbf{K}}(s),\{(t, s) \in$ $\left.\mathbb{R}^{2} \mid 0 \leq s \leq t\right\} \rightarrow \mathcal{L}_{b}\left(\mathcal{S}^{*}\left(\mathbb{R}^{d}\right), \mathcal{S}^{*}\left(\mathbb{R}^{d}\right)\right)$, is continuous. Hence, for each $C>0$, $\{T(t-s) \tilde{\mathbf{K}}(s) \mid 0 \leq s \leq t \leq C\}$ is an equicontinuous subset of $\mathcal{L}\left(\mathcal{S}^{*}\left(\mathbb{R}^{d}\right), \mathcal{S}^{*}\left(\mathbb{R}^{d}\right)\right)$. Employing this fact together with (6.23) and the semigroup property of $T(t)$, one can prove that $t \mapsto \mathbf{Q}(t),[0, \infty) \rightarrow \mathcal{L}_{b}\left(\mathcal{S}^{*}\left(\mathbb{R}^{d}\right), \mathcal{S}^{*}\left(\mathbb{R}^{d}\right)\right)$, is continuous. Now, reproducing the proof of [17, Lemma 7.15$]$ verbatim, one gets the following result.

Lemma 6.11 The mapping $t \mapsto \mathbf{Q}(t)$ belongs to $C^{\infty}\left([0, \infty) ; \mathcal{L}_{b}\left(\mathcal{S}^{*}\left(\mathbb{R}^{d}\right), \mathcal{S}^{*}\left(\mathbb{R}^{d}\right)\right)\right)$.

Denoting the Weyl symbol of $\mathbf{Q}(t)$ by $Q(t, w)$, this lemma together with the property of symbols of operators in $\mathcal{L}\left(\mathcal{S}^{*}\left(\mathbb{R}^{d}\right), \mathcal{S}^{*}\left(\mathbb{R}^{d}\right)\right)$ ) (cf. [18, Propositions 2 and 3$]$ ) imply:

Corollary 6.12 The mapping $t \mapsto Q(t, \cdot)$ belongs to $C^{\infty}\left([0, \infty) ; \mathcal{S}^{*}\left(\mathbb{R}^{2 d}\right)\right)$.

Notice that (6.1) together with $a(w) / \ln |w| \rightarrow+\infty$, as $|w| \rightarrow \infty$, ensures that $(\mathbf{u}(t))^{w}$ is trace-class for each $t>0$ (cf. [13, Theorem 4.4.21, p. 190]). Now, Lemma 6.11 ensures that $T(t)$ is also trace-class for $t>0$. As $T(t)$ are self-adjoint, we conclude $\operatorname{Tr} T(t)=\sum_{j=0}^{\infty} e^{-t \lambda_{j}}$. Thus,

$$
\sum_{j=0}^{\infty} e^{-t \lambda_{j}}=\frac{1}{(2 \pi)^{d}} \int_{\mathbb{R}^{2 d}} u(t, w) d w-\frac{1}{(2 \pi)^{d}} \int_{\mathbb{R}^{2 d}} Q(t, w) d w, \quad t>0
$$


The second integral is $O(1)$ as $t \rightarrow 0^{+}$(because of Corollary 6.12). Fix $n>d / \rho$, $n \in \mathbb{Z}_{+}$. Since $u_{0}(t, w)=e^{-t b(w)}$ and $b(w)=a(w)$ for $w$ outside of a compact neighbourhood of the origin, we have

$$
\begin{aligned}
\sum_{j=0}^{\infty} e^{-t \lambda_{j}}= & \frac{1}{(2 \pi)^{d}} \int_{\mathbb{R}^{2 d}} e^{-t a(w)} d w+\frac{1}{(2 \pi)^{d}} \sum_{j=1}^{n-1} \int_{\mathbb{R}^{2 d}} u_{j}(t, w) d w \\
& +\frac{1}{(2 \pi)^{d}} \int_{\mathbb{R}^{2 d}}\left(u(t, w)-\sum_{j=0}^{n-1} u_{j}(t, w)\right) d w+O(1), t \rightarrow 0^{+} .
\end{aligned}
$$

In view of the second estimate in Lemma 6.2 (specialised for $n=0$ and $\alpha=0$ ), the very last integral is $O(1)$ as $t \rightarrow 0^{+}$. Lemma $6.1 \mathrm{implies}$ that there exists $C^{\prime}>0 \mathrm{such}$ that $\left|u_{j}(t, w)\right| \leq C e^{-\frac{t}{4} b(w)}\langle w\rangle^{-2 \rho}$, for all $w \in \mathbb{R}^{2 d}, t \geq 0, j=1, \ldots, n-1$. Using again $b=a$ except in a compact neighbourhood of 0 , we have

$$
\sum_{j=0}^{\infty} e^{-t \lambda_{j}}=\frac{1}{(2 \pi)^{d}} \int_{\mathbb{R}^{2 d}} e^{-t a(w)} d w+O\left(\int_{\mathbb{R}^{2 d}} \frac{e^{-\frac{t}{4} a(w)}}{\langle w\rangle^{2 \rho}} d w\right)+O(1), \quad t \rightarrow 0^{+}
$$

We claim

$$
\lim _{t \rightarrow 0^{+}} \int_{\mathbb{R}^{2 d}} \frac{e^{-t a(w) / 4}}{\langle w\rangle^{2 \rho}} d w=\infty
$$

To verify it, first notice that $a \in \Gamma_{A_{p}, \rho}^{*, \infty}\left(\mathbb{R}^{2 d}\right)$ implies that there are $m, C>0$ (resp. for every $m>0$ there exists $C>0$ ) such that $a(w) \leq C e^{M(m|w|)}, \forall w \in \mathbb{R}^{2 d}$. Using this estimate (in the Roumieu case we can take $m=1$ with the corresponding $C>0$ ) and polar coordinates, we have

$$
\begin{aligned}
\int_{\mathbb{R}^{2 d}} \frac{e^{-t a(w) / 4}}{\langle w\rangle^{2 \rho}} d w & \geq \int_{\mathbb{S}^{2 d-1}} \int_{0}^{\infty} \exp \left(-\frac{t C e^{M(m r)}}{4}\right) \frac{r^{2 d-1}}{\left(1+r^{2}\right)^{\rho}} d r d \vartheta \\
& \geq \frac{2 \pi^{d}}{(d-1) !} \int_{1}^{\infty} \exp \left(-\frac{t C e^{M(m r)}}{4}\right) \frac{r}{1+r^{2}} d r
\end{aligned}
$$

Monotone convergence implies that the very last integral tends to $\infty$ as $t \rightarrow 0^{+}$. We have shown:

Theorem 6.13 Let a be a hypoelliptic real-valued symbol in $\Gamma_{A_{p}, \rho}^{*, \infty}\left(\mathbb{R}^{2 d}\right)$ such that

$$
\lim _{|w| \rightarrow \infty} \frac{a(w)}{\ln |w|}=\infty
$$


Then

$$
\sum_{j=0}^{\infty} e^{-t \lambda_{j}}=\frac{1}{(2 \pi)^{d}} \int_{\mathbb{R}^{2 d}} e^{-t a(w)} d w+O\left(\int_{\mathbb{R}^{2 d}} \frac{e^{-\frac{t}{4} a(w)}}{\langle w\rangle^{2 \rho}} d w\right), t \rightarrow 0^{+}
$$

The next remark shows that (6.25) remains valid for hypoelliptic symbols of finite order.

Remark 6.14 Let $a \in \Gamma_{\rho}^{m}\left(\mathbb{R}^{2 d}\right)$ be a hypoelliptic real-valued symbol such that $a(w) \geq$ $c\langle w\rangle^{\delta}$ for some $\delta>0, \forall|w| \geq c$, and consider its heat parametrix $(\mathbf{u}(t))^{w}=(u(t, \cdot))^{w}$ as constructed in Remark 6.4 and the $C_{0}$-semigroup $\{T(t)\}_{t \geq 0}$ as given by (6.4). The fact $t \mapsto T(t) \in C^{\infty}\left([0, \infty) ; \mathcal{L}_{b}\left(\mathcal{S}\left(\mathbb{R}^{d}\right), \mathcal{S}\left(\mathbb{R}^{d}\right)\right)\right)$ can be proved far more easily in the distributional setting. To verify this, first notice that $\left(a^{w}\right)^{j}$ is hypoelliptic for each $j \in \mathbb{Z}_{+}$and denote its symbol by $a_{j} \in \Gamma_{\rho}^{j m}\left(\mathbb{R}^{2 d}\right)$. Clearly $\left|a_{j}(w)\right| \geq\langle w\rangle^{\delta j}$ away the origin. For each $\varphi \in \mathcal{S}\left(\mathbb{R}^{d}\right), t \geq 0$ and $j \in \mathbb{Z}_{+}$, we have $\left(a^{w}\right)^{j} T(t) \varphi=$ $T(t)\left(a^{w}\right)^{j} \varphi \in L^{2}\left(\mathbb{R}^{d}\right)$. Because of [13, Theorem 2.1.16, p. 76], $T(t) \varphi$ belongs to all Sobolev spaces $H_{\Gamma}^{k}\left(\mathbb{R}^{d}\right), k \in \mathbb{Z}_{+}$, and thus $T(t) \varphi \in \mathcal{S}\left(\mathbb{R}^{d}\right)$. Now, the closed graph theorem yields $T(t) \in \mathcal{L}\left(\mathcal{S}\left(\mathbb{R}^{d}\right), \mathcal{S}\left(\mathbb{R}^{d}\right)\right), t \geq 0$. Since $\mathcal{S}\left(\mathbb{R}^{d}\right)=\lim _{k \rightarrow \infty} H_{\Gamma}^{k}\left(\mathbb{R}^{d}\right)$, in order to prove that $t \mapsto T(t)$ is right continuous at $t_{0}$ it is enough to prove that for each $k \in \mathbb{Z}_{+}, \varepsilon>0$ and bounded subset $B$ of $\mathcal{S}\left(\mathbb{R}^{d}\right)$, there exists $\eta>0$ such that $\left\|T(t) \varphi-T\left(t_{0}\right) \varphi\right\|_{H_{\Gamma}^{k}} \leq \varepsilon, \forall t \in\left(t_{0}, t_{0}+\eta\right), \forall \varphi \in B$. The a priori estimate in [13, Theorem 2.1.16, p. 76] yields that there exist $C>0$ and $j \in \mathbb{Z}_{+}$such that $\left\|T(t) \varphi-T\left(t_{0}\right) \varphi\right\|_{H_{\Gamma}^{k}}$

$$
\leq C\left\|T\left(t_{0}\right)\right\|_{\mathcal{L}_{b}\left(L^{2}\left(\mathbb{R}^{d}\right)\right)}\left(\left\|\left(T\left(t-t_{0}\right)-\mathrm{Id}\right)\left(a^{w}\right)^{j} \varphi\right\|_{L^{2}}+\left\|\left(T\left(t-t_{0}\right)-\mathrm{Id}\right) \varphi\right\|_{L^{2}}\right) .
$$

Since $T(t) \rightarrow$ Id in $\mathcal{L}_{p}\left(L^{2}\left(\mathbb{R}^{d}\right), L^{2}\left(\mathbb{R}^{d}\right)\right.$ ) (by the Banach-Steinhaus theorem; $\{T(t)\}_{t \geq 0}$ is a $C_{0}$-semigroup) and $B$ and $\left(a^{w}\right)^{j}(B)$ are precompact in $\mathcal{S}\left(\mathbb{R}^{d}\right)$ and hence also in $L^{2}\left(\mathbb{R}^{d}\right)$, we obtain that $t \mapsto T(t)$ is right continuous at $t_{0}$. Similarly, one proves that it is left continuous. The same a priori estimate proves that the set $H=\left\{\left(t-t_{0}\right)^{-1}\left(T(t)-T\left(t_{0}\right)\right) \mid t \in\left(\left[t_{0}-1, t_{0}+1\right] \backslash\left\{t_{0}\right\}\right) \cap[0, \infty)\right\}$ is bounded in $\mathcal{L}_{\sigma}\left(\mathcal{S}\left(\mathbb{R}^{d}\right), \mathcal{S}\left(\mathbb{R}^{d}\right)\right)$, hence equicontinuous. Again, the same a priori estimate proves $\left(t-t_{0}\right)^{-1}\left(T(t)-T\left(t_{0}\right)\right) \rightarrow-a^{w} T\left(t_{0}\right)$ in $\mathcal{L}_{\sigma}\left(\mathcal{S}\left(\mathbb{R}^{d}\right), \mathcal{S}\left(\mathbb{R}^{d}\right)\right)$ and, as $H$ is equicontinuous, the Banach-Steinhaus theorem [19, Theorem 4.5, p. 85] gives the limit in the topology of precompact convergence. As $\mathcal{S}\left(\mathbb{R}^{d}\right)$ is Montel, the limit holds in the strong topology. This immediately yields $t \mapsto T(t) \in C^{\infty}\left([0, \infty) ; \mathcal{L}_{b}\left(\mathcal{S}\left(\mathbb{R}^{d}\right), \mathcal{S}\left(\mathbb{R}^{d}\right)\right)\right)$. Now one can obtain in the same way as above the validity of Lemma 6.11 and Corollary 6.12 in this case as well (of course, with $\mathcal{S}\left(\mathbb{R}^{d}\right)$ and $\mathcal{S}^{\prime}\left(\mathbb{R}^{d}\right)$ in place of $\mathcal{S}^{*}\left(\mathbb{R}^{d}\right)$ and $\left.\mathcal{S}^{* *}\left(\mathbb{R}^{d}\right)\right)$.

Using the estimates for $u(t, w)$ and $u_{j}(t, w)$ given in Remark 6.4, one readily obtains (6.24) and the asymptotic estimate (6.25) from Theorem 6.13 in the finite order case too. 


\section{The Weyl asymptotic formula for infinite order $\Psi$ DOs. Part II: proofs of the main results}

We now present the proofs of Theorems 5.1, 5.2, 5.4, and Corollary 5.3. In the sequel, we also use Vinogradov's notation for $O$-estimates, namely, $g_{1}(t) \ll g_{2}(t)$ as an alternative way of writing $g_{1}(t)=O\left(g_{2}(t)\right)$.

We first make some comments that apply to all cases simultaneously. A preliminary observation is that $f(y) / y^{\delta} \rightarrow \infty$ as $y \rightarrow \infty$ for any $0<\delta<$ $\liminf _{y \rightarrow \infty} y f^{\prime}(y) / f(y)$ as follows by integrating (5.13) which holds in the three cases. It then follows from (5.3), (5.8), or (5.12) that $a(w) /|w|^{\delta} \rightarrow \infty$ as $w \rightarrow \infty$. Incidentally, this also implies that $f^{\prime}(y)>0$ a.e. on $\left[Y_{1}, \infty\right)$, for some large enough $Y_{1} \geq Y$ and additionally $f(y)>1$ on $\left[Y_{1}, \infty\right)$. Without loss of generality, we may assume $Y_{1}=Y>1$. We conclude that $\sigma$ is absolutely continuous on every compact interval contained in $[f(Y), \infty)$. We extend $\sigma$ to $[0, f(Y)]$ as a positive non-decreasing absolutely continuous function with $\sigma(\lambda)=1$ near $\lambda=0$. Note also that $\sigma(\lambda) \rightarrow \infty$ as $\lambda \rightarrow \infty$. We now derive some regular variation properties of $\sigma$.

For Theorems 5.1 and 5.2, and Corollary 5.3, we combine (5.2) and (5.7) into

$$
\lim _{y \rightarrow \infty} \frac{y f^{\prime}(y)}{f(y)}=\beta \in(0, \infty]
$$

Let us verify that (7.1) implies that $\sigma$ is a Karamata regular varying function [1] with index of regular variation $2 d / \beta(=0$ if $\beta=\infty)$, that is, that

$$
\lim _{\lambda \rightarrow \infty} \frac{\sigma(\alpha \lambda)}{\sigma(\lambda)}=\alpha^{\frac{2 d}{\beta}}
$$

uniformly for $\alpha$ in compact subsets of $(0, \infty)$. In fact, we have that

$$
\eta(\lambda)=\frac{\lambda \sigma^{\prime}(\lambda)}{\sigma(\lambda)}=2 d \frac{f\left(f^{-1}(\lambda)\right)}{f^{-1}(\lambda) f^{\prime}\left(f^{-1}(\lambda)\right)} \rightarrow \frac{2 d}{\beta}, \quad \lambda \rightarrow \infty,
$$

and

$$
\sigma(\lambda)=\exp \left(\int_{0}^{\lambda} \frac{\eta(t)}{t} d t\right)
$$

for all $\lambda$ (note that $\eta(t)$ vanishes for $t$ near 0 ). This easily yields (7.2).

Similarly, the hypothesis (5.13) and the fact that $\sigma$ is increasing imply that there are $v, C_{1}>0$ such that

$$
\sigma(\alpha \lambda) / \sigma(\lambda) \leq C_{1}(\alpha+1)^{v}, \quad \forall \alpha, \lambda>0
$$

In fact, we may take any $v>0$ such that $2 d / v<\beta^{\prime}=\liminf _{y \rightarrow \infty} y f^{\prime}(y) / f(y)$. For $\nu$ in this range, the inequality can be refined for large $\lambda$. Indeed, there is $\lambda_{0}=\lambda_{0}(v)$ such that

$$
\sigma(\alpha \lambda) / \sigma(\lambda) \leq \alpha^{v}, \quad \forall \alpha \geq 1, \lambda \geq \lambda_{0} .
$$


The next starting point is the formula (6.25) from Theorem 6.13, which holds under all our three sets of hypotheses (see Remark 6.14 for the finite order case). As there are only finitely many possibly negative eigenvalues, we obtain [cf. (6.24)]

$$
\int_{0}^{\infty} e^{-t \lambda} d N(\lambda)=\frac{1}{(2 \pi)^{d}} \int_{\mathbb{R}^{2 d}} e^{-t a(w)} d w+O\left(\int_{\mathbb{R}^{2 d}} \frac{e^{-t a(w) / 4}}{\langle w\rangle^{2 \rho}} d w\right), \quad t \rightarrow 0^{+}
$$

Proof of Theorem 5.1 Let $\varepsilon>0$ be arbitrary but fixed and set

$$
C^{\prime}=\frac{1}{2 d} \int_{\mathbb{S}^{2 d-1}} \frac{d \vartheta}{(\Phi(\vartheta))^{2 d}}
$$

Using polar coordinates and the lower bound from (5.3), we have that

$$
\begin{aligned}
\int_{\mathbb{R}^{2 d}} e^{-t a(w)} d w & \leq \int_{\mathbb{S}^{2 d-1}} \int_{B_{\varepsilon}}^{\infty} r^{2 d-1} e^{-c_{\varepsilon} t f((1-\varepsilon) r \Phi(\vartheta))} d r d \vartheta+\int_{|w| \leq B_{\varepsilon}} e^{-t a(w)} d w \\
& =\int_{\mathbb{S}^{2 d-1}} \int_{B_{\varepsilon}}^{\infty} r^{2 d-1} e^{-c_{\varepsilon} t f((1-\varepsilon) r \Phi(\vartheta))} d r d \vartheta+O_{\varepsilon}(1) \\
& =(1-\varepsilon)^{-2 d} C^{\prime} \int_{f(Y)}^{\infty} e^{-c_{\varepsilon} t \lambda} \sigma^{\prime}(\lambda) d \lambda+O_{\varepsilon}(1) \\
& =(1-\varepsilon)^{-2 d} C^{\prime} \int_{0}^{\infty} e^{-\lambda} \sigma\left(\lambda /\left(c_{\varepsilon} t\right)\right) d \lambda+O_{\varepsilon}(1)
\end{aligned}
$$

where we have used the change of variables $\lambda=f((1-\varepsilon) r \Phi(\vartheta))$ which gives

$$
r^{2 d-1} d r=\frac{1}{2 d}\left(\frac{1}{(1-\varepsilon) \Phi(\vartheta)}\right)^{2 d} \sigma^{\prime}(\lambda) d \lambda
$$

Since $\sigma$ is slowly varying (i.e. $\sigma(\alpha \lambda) / \sigma(\lambda) \rightarrow 1$ as $\lambda \rightarrow \infty$ ),

$$
\int_{0}^{\infty} e^{-\lambda} \sigma\left(\lambda /\left(c_{\varepsilon} t\right)\right) d \lambda \sim \sigma(1 / t), \quad t \rightarrow 0^{+},
$$

as follows from the Lebesgue dominated convergence theorem [the bound (7.3) holds here for every $v>0$ and $C_{1}$ depending only on $v$. Thus,

$$
\limsup _{t \rightarrow 0^{+}} \frac{1}{\sigma(1 / t)} \int_{\mathbb{R}^{2 d}} e^{-t a(w)} d w \leq(1-\varepsilon)^{-2 d} C^{\prime},
$$

because $\sigma(1 / t) \rightarrow \infty$. But we can now take $\varepsilon \rightarrow 0^{+}$to conclude

$$
\limsup _{t \rightarrow 0^{+}} \frac{1}{\sigma(1 / t)} \int_{\mathbb{R}^{2 d}} e^{-t a(w)} d w \leq C^{\prime}
$$


Similarly,

$$
\liminf _{t \rightarrow 0^{+}} \frac{1}{\sigma(1 / t)} \int_{\mathbb{R}^{2 d}} e^{-t a(w)} d w \geq C^{\prime}
$$

therefore,

$$
\frac{1}{(2 \pi)^{d}} \int_{\mathbb{R}^{2 d}} e^{-t a(w)} d w \sim \frac{C^{\prime}}{(2 \pi)^{d}} \sigma(1 / t), \quad t \rightarrow 0^{+} .
$$

On the other hand, a small computation along the same lines as the above one shows that

$$
\int_{\mathbb{R}^{2 d}} \frac{e^{-t a(w) / 4}}{\langle w\rangle^{2 \rho}} d w \ll \sigma(1 / t)^{1-\frac{\rho}{d}}=o(\sigma(1 / t)) .
$$

Inserting all this into (7.5), we conclude

$$
\int_{0}^{\infty} e^{-t \lambda} d N(\lambda) \sim \frac{C^{\prime}}{(2 \pi)^{d}} \sigma\left(\frac{1}{t}\right), \quad t \rightarrow 0^{+},
$$

and (5.4) follows from the well known Karamata Tauberian theorem [1, Theorem 1.7.1, p. 37] (see also [11, Theorem 8.1, p. 193]).

Using (5.4) and employing a classical argument (see e.g. [13, Proposition 4.6.4, p. 198], the same proof works fine in our case), we obtain that

$$
\sigma\left(\lambda_{j}\right) \sim \frac{(2 \pi)^{d}}{C^{\prime}} j, \quad j \rightarrow \infty
$$

Notice that (5.5) is equivalent to (7.8). Finally, (5.6) follows from (5.5) and

$$
\frac{f(\alpha y)}{f\left(\alpha^{\prime} y\right)}=\exp \left(\int_{\alpha^{\prime} y}^{\alpha y} \frac{f^{\prime}(t)}{f(t)} d t\right) \rightarrow \infty, \quad y \rightarrow \infty,
$$

valid for every $\alpha^{\prime}<\alpha$ because of (5.2). This completes the proof of Theorem 5.1.

Proof of Theorem 5.2 Pick $\varepsilon>0$ and find $B$ so large that

$$
(1-\varepsilon) f(r) \Phi(\vartheta) \leq a(r \vartheta) \leq(1+\varepsilon) f(r) \Phi(\vartheta)
$$

for all $\vartheta \in \mathbb{S}^{2 d-1}$ and $r>B$. Note that $\Phi$ is continuous and thus $\Phi(\vartheta)$ stays on a compact subset of $(0, \infty)$. Using that (7.2) is valid uniformly for $\alpha$ on compact subsets of $(0, \infty)$, we then obtain,

$$
\begin{aligned}
\frac{1}{\sigma(1 / t)} \int_{\mathbb{R}^{2 d}} e^{-t a(w)} d w & \leq \frac{1}{\sigma(1 / t)} \int_{\mathbb{S}^{2 d-1}} \int_{0}^{\infty} e^{-(1-\varepsilon) t \Phi(\vartheta) f(r)} r^{2 d-1} d r d \vartheta+o_{\varepsilon}(1) \\
& =\frac{1}{2 d} \int_{0}^{\infty} e^{-\lambda}\left(\int_{\mathbb{S}^{2 d-1}} \frac{\sigma(\lambda /((1-\varepsilon) \Phi(\vartheta) t))}{\sigma(1 / t)} d \vartheta\right) d \lambda+o_{\varepsilon}(1)
\end{aligned}
$$




$$
\begin{aligned}
& =\frac{\int_{0}^{\infty} e^{-\lambda} \lambda^{2 d / \beta} d \lambda}{2 d(1-\varepsilon)^{2 d / \beta}}\left(\int_{\mathbb{S}^{2 d-1}} \frac{d \vartheta}{(\Phi(\vartheta))^{2 d / \beta}}\right)+o_{\varepsilon}(1) \\
& =\frac{\Gamma\left(1+\frac{2 d}{\beta}\right)}{2 d(1-\varepsilon)^{2 d / \beta}} \int_{\mathbb{S}^{2 d-1}} \frac{d \vartheta}{(\Phi(\vartheta))^{2 d / \beta}}+o_{\varepsilon}(1), \quad t \rightarrow 0^{+} .
\end{aligned}
$$

Taking first $t \rightarrow 0^{+}$and then $\varepsilon \rightarrow 0^{+}$, we conclude that

$$
\limsup _{t \rightarrow 0^{+}} \frac{1}{\sigma(1 / t)} \int_{\mathbb{R}^{2 d}} e^{-t a(w)} d w \leq \frac{\Gamma\left(1+\frac{2 d}{\beta}\right)}{2 d} \int_{\mathbb{S}^{2 d-1}} \frac{d \vartheta}{(\Phi(\vartheta))^{2 d / \beta}}
$$

The estimate (7.7) remains valid in this case too. A similar analysis for the limit inferior, together with (7.5) and (7.7), leads to

$$
\int_{0}^{\infty} e^{-t \lambda} d N(\lambda) \sim \sigma(1 / t) \frac{\pi \Gamma\left(1+\frac{2 d}{\beta}\right)}{(2 \pi)^{d+1} d} \int_{\mathbb{S}^{2 d-1}} \frac{d \vartheta}{(\Phi(\vartheta))^{2 d / \beta}}, \quad t \rightarrow 0^{+}
$$

We can apply once again the Karamata Tauberian theorem $[1,11]$ to conclude that (5.9) holds.

The classical argument quoted above in the proof of Theorem 5.1 easily gives $\sigma\left(\lambda_{j}\right) \sim j / C, j \rightarrow \infty$, with $C=d^{-1}(2 \pi)^{-d-1} \pi \int_{\mathbb{S}^{2 d-1}}(\Phi(\vartheta))^{-2 d / \beta} d \vartheta$. This immediately implies $(j / C)^{\frac{1}{2 d}} \sim f^{-1}\left(\lambda_{j}\right)$, as $j \rightarrow \infty$. Note that (5.7) yields that $f$ is regularly varying of index $\beta$, i.e., $f(\alpha \lambda) \sim \alpha^{\beta} f(\lambda), \lambda \rightarrow \infty$, uniformly for $\alpha>0$ on compacts of $(0, \infty)$. Using this, $\lambda_{j}=f\left((j / C)^{\frac{1}{2 d}}(1+o(1))\right) \sim C^{-\frac{\beta}{2 d}} f\left(j^{\frac{1}{2 d}}\right)$, which is $(5.10)$.

Proof of Corollary 5.3 We only give the proof under the assumptions of Theorem 5.1, the proof of this corollary with the hypotheses from Theorem 5.2 is similar and the details are therefore left to the reader. By Theorem 5.1, we only need to show that

$$
\int_{a(w)<\lambda} d w \sim C^{\prime} \sigma(\lambda), \quad \lambda \rightarrow \infty
$$

where $C^{\prime}$ is given by (7.6). We show that

$$
\limsup _{\lambda \rightarrow \infty} \frac{1}{\sigma(\lambda)} \int_{a(w)<\lambda} d w \leq C^{\prime}
$$

one treats analogously the limit inferior to obtain the desired result and we thus omit the calculation. Fixing $\varepsilon>0$, using the lower bound from (5.3) (choose $B_{\varepsilon}>Y$ ), polar coordinates, and (7.2), we have 


$$
\begin{aligned}
\limsup _{\lambda \rightarrow \infty} \frac{1}{\sigma(\lambda)} \int_{a(w)<\lambda} d w & \leq \limsup _{\lambda \rightarrow \infty} \frac{1}{\sigma(\lambda)} \int_{\mathbb{S}^{2 d-1}} \int_{\left\{r \mid B_{\varepsilon}<r, a(r \vartheta)<\lambda\right\}} r^{2 d-1} d r d \vartheta \\
& \leq \lim _{\lambda \rightarrow \infty} \frac{1}{\sigma(\lambda)} \int_{\mathbb{S}^{2 d-1}} \int_{B_{\varepsilon}}^{(1+\varepsilon) f^{-1}\left(\lambda / c_{\varepsilon}\right) / \Phi(\vartheta)} r^{2 d-1} d r d \vartheta \\
& =(1+\varepsilon)^{2 d} C^{\prime} \lim _{\lambda \rightarrow \infty} \frac{\sigma\left(\lambda / c_{\varepsilon}\right)}{\sigma(\lambda)}=(1+\varepsilon)^{2 d} C^{\prime} .
\end{aligned}
$$

The result now follows by taking $\varepsilon \rightarrow 0^{+}$.

Proof of Theorem 5.4 The lower bound (5.12) still applies to show (7.7). Combining this with the asymptotic estimate (7.5), we obtain

$$
\int_{0}^{\infty} e^{-t \lambda} d N(\lambda)=\frac{1}{(2 \pi)^{d}} \int_{\mathbb{R}^{2 d}} e^{-t a(w)} d w+o(\sigma(1 / t)), \quad t \rightarrow 0^{+} .
$$

When either (5.13) or (5.16) holds, fix $v>2 d / \beta^{\prime}$ and find $\lambda_{0}>0$ such that (7.4) holds. For $0<t \leq 1 / \lambda_{0}$, we deduce from (5.12) that

$$
\begin{aligned}
\int_{\mathbb{R}^{2 d}} e^{-t a(w)} d w & \leq \int_{\mathbb{S}^{2 d-1}} \int_{B \leq r} e^{-C t f(r)} r^{2 d-1} d r d \vartheta+O_{\nu}(1) \\
& =\frac{\pi^{d}}{d !} \int_{0}^{\infty} e^{-\lambda} \sigma(\lambda /(C t)) d \lambda+O_{\nu}(1) .
\end{aligned}
$$

If (5.13) holds, then the monotonicity of $\sigma$ together with (7.4) yields

$$
\begin{aligned}
\int_{\mathbb{R}^{2 d}} e^{-t a(w)} d w & \leq \frac{\pi^{d}}{d !} \int_{0}^{C} e^{-\lambda} \sigma(\lambda /(C t)) d \lambda+\frac{\pi^{d}}{d !} \int_{C}^{\infty} e^{-\lambda} \sigma(\lambda /(C t)) d \lambda+O_{v}(1) \\
& \leq \frac{\pi^{d}}{d !} \sigma(1 / t)+\frac{\pi^{d}}{d !} \sigma(1 / t) \int_{0}^{\infty} e^{-\lambda}\left(\frac{\lambda}{C}\right)^{v} d \lambda+O_{v}(1) \\
& \leq \frac{\pi^{d}}{d !} \sigma(1 / t)\left(1+\frac{\Gamma(1+v)}{C^{v}}\right)+O_{v}(1) .
\end{aligned}
$$

Using (7.9) and keeping still $t \leq 1 / \lambda_{0}$,

$$
\begin{aligned}
N(1 / t)-N(0) & =\int_{0}^{1 / t} d N(\lambda) \leq e \int_{0}^{1 / t} e^{-t \lambda} d N(\lambda) \leq e \int_{0}^{\infty} e^{-t \lambda} d N(\lambda) \\
& \leq \frac{e}{2^{d} d !}\left(1+\frac{\Gamma(1+v)}{C^{\nu}}\right) \sigma(1 / t)\left(1+o_{\nu}(1)\right) .
\end{aligned}
$$

Dividing through by $\sigma(1 / t)$, taking the limit superior as $t \rightarrow 0^{+}$, and letting then $v \rightarrow 2 d / \beta^{\prime}$, we obtain the estimate (5.14). The lower bound (5.15) easily follows by inserting $\lambda=\lambda_{j}$ in (5.14) and the fact $N\left(\lambda_{j}\right) \geq j, \forall j \in \mathbb{N}$. If (5.16) holds, we divide 
(7.10) by $\sigma(1 / t)$ and take the limit superior as $t \rightarrow 0^{+}$. Because of (7.2) we have

$$
\limsup _{t \rightarrow 0^{+}} \frac{1}{\sigma(1 / t)} \int_{\mathbb{R}^{2 d}} e^{-t a(w)} d w \leq \frac{\pi^{d} \Gamma\left(1+2 d / \beta^{\prime}\right)}{d ! C^{2 d / \beta^{\prime}}} .
$$

Now, the same technique as before yields the rest of the assertions of the theorem.

Open Access This article is distributed under the terms of the Creative Commons Attribution 4.0 International License (http://creativecommons.org/licenses/by/4.0/), which permits unrestricted use, distribution, and reproduction in any medium, provided you give appropriate credit to the original author(s) and the source, provide a link to the Creative Commons license, and indicate if changes were made.

\section{Appendix}

We collect here some important facts concerning symbolic calculus and the construction of parametrices for operators with symbols in $\Gamma_{A_{p}, \rho}^{*, \infty}\left(\mathbb{R}^{2 d}\right)$. We start with the following continuity result.

Proposition 8.1 [17, Proposition 3.1] For each $\tau \in \mathbb{R}$, the bilinear mapping $(a, \varphi) \mapsto$ $\operatorname{Op}_{\tau}(a) \varphi, \Gamma_{A_{p}, \rho}^{*, \infty}\left(\mathbb{R}^{2 d}\right) \times \mathcal{S}^{*}\left(\mathbb{R}^{d}\right) \rightarrow \mathcal{S}^{*}\left(\mathbb{R}^{d}\right)$, is hypocontinuous and it extends to the hypocontinuous bilinear mapping $(a, T) \mapsto \mathrm{Op}_{\tau}(a) T, \Gamma_{A_{p}, \rho}^{*, \infty}\left(\mathbb{R}^{2 d}\right) \times \mathcal{S}^{*}\left(\mathbb{R}^{d}\right) \rightarrow$ $\mathcal{S}^{*}\left(\mathbb{R}^{d}\right)$. The mappings $a \mapsto \mathrm{Op}_{\tau}(a), \Gamma_{A_{p}, \rho}^{*, \infty}\left(\mathbb{R}^{2 d}\right) \rightarrow \mathcal{L}_{b}\left(\mathcal{S}^{*}\left(\mathbb{R}^{d}\right), \mathcal{S}^{*}\left(\mathbb{R}^{d}\right)\right)$, $\Gamma_{A_{p}, \rho}^{*, \infty}\left(\mathbb{R}^{2 d}\right) \rightarrow \mathcal{L}_{b}\left(\mathcal{S}^{* *}\left(\mathbb{R}^{d}\right), \mathcal{S}^{*}\left(\mathbb{R}^{d}\right)\right)$ are continuous.

As we mentioned before, changing the quantisation always results in operators with symbols in $\Gamma_{A_{p}, \rho}^{*, \infty}\left(\mathbb{R}^{2 d}\right)$ modulo $*$-regularising operators (see $[17,18]$ ).

The composition of two Weyl quantisation is again a $\Psi$ DO (modulo a $*$-regularising operator) with Weyl symbol "given" by their \#-product. More precisely

Theorem 8.2 [17, Theorem 4.2] Let $U_{1}, U_{2} \subseteq F S_{A_{p}, \rho}^{*, \infty}\left(\mathbb{R}^{2 d} ; B\right)$ be such that $U_{1} \precsim f_{1}$ and $U_{2} \precsim f_{2}$ in $F S_{A_{p}, \rho}^{*, \infty}\left(\mathbb{R}^{2 d} ; B\right)$ for some continuous positive functions $f_{1}$ and $f_{2}$ with ultrapolynomial growth of class $*$. Then:

(i) $U_{1} \# U_{2} \precsim f_{1} f_{2}$ in $F S_{A_{p}, \rho}^{*, \infty}\left(\mathbb{R}^{2 d} ; B\right)$.

(ii) Let $V_{k} \precsim f_{k} U_{k}$, with $\Sigma_{k}: U_{k} \rightarrow V_{k}$ the surjective mapping, $k=1,2$. There exists $R>0$, which can be chosen arbitrarily large, such that

$$
\left.\begin{array}{l}
\left\{\mathrm{Op}_{1 / 2}\left(\Sigma_{1}\left(\sum_{j} a_{j}\right)\right) \mathrm{Op}_{1 / 2}\left(\Sigma_{2}\left(\sum_{j} b_{j}\right)\right)\right. \\
-\mathrm{Op}_{1 / 2}\left(R\left(\sum_{j} a_{j} \# \sum_{j} b_{j}\right)\right) \mid \sum_{j} a_{j} \in U_{1}, \sum_{j} b_{j} \in U_{2}
\end{array}\right\}
$$


is an equicontinuous subset of $\mathcal{L}\left(\mathcal{S}^{*}\left(\mathbb{R}^{d}\right), \mathcal{S}^{*}\left(\mathbb{R}^{d}\right)\right)$ and

$$
\left\{R\left(\sum_{j} a_{j} \# \sum_{j} b_{j}\right) \mid \sum_{j} a_{j} \in U_{1}, \sum_{j} b_{j} \in U_{2}\right\} \precsim f_{1} f_{2} U_{1} \# U_{2} .
$$

Corollary 8.3 [17, Corollary 4.3] Let $U_{1}, U_{2} \subseteq F S_{A_{p}, \rho}^{*, \infty}\left(\mathbb{R}^{2 d} ; B\right)$ with $U_{1} \precsim f_{1}$ and $U_{2} \precsim f_{2}$ for some continuous positive functions of ultrapolynomial growth of class *. For $\sum_{j} a_{j} \in U_{1}$ and $\sum_{j} b_{j} \in U_{2}$ denote $\sum_{j} c_{j, a, b}=\sum_{j} a_{j} \# \sum_{j} b_{j} \in U_{1} \# U_{2}$. Then, there exists $R>0$, which can be chosen arbitrarily large, such that

$$
\left\{a^{w} b^{w}-c^{w} \mid a=R\left(\sum_{j} a_{j}\right), b=R\left(\sum_{j} b_{j}\right), c=R\left(\sum_{j} c_{j, a, b}\right)\right\}
$$

is an equicontinuous subset of $\mathcal{L}\left(\mathcal{S}^{*}\left(\mathbb{R}^{d}\right), \mathcal{S}^{*}\left(\mathbb{R}^{d}\right)\right)$ and $(8.1)$ holds.

Remark 8.4 Corollary 8.3 is also applicable when $U_{1}$ and $U_{2}$ are bounded subsets of $\Gamma_{A_{p}, \rho}^{\left(M_{p}\right), \infty}\left(\mathbb{R}^{2 d} ; m\right)$ for some $m>0$ (resp. of $\Gamma_{A_{p}, \rho}^{\left\{M_{p}\right\}, \infty}\left(\mathbb{R}^{2 d} ; h\right)$ for some $h>0$ ). In this case, the corollary reads: there exists $R>0$, which can be chosen arbitrary large, such that $\left\{a^{w} b^{w}-\mathrm{Op}_{1 / 2}(R(a \# b)) \mid a \in U_{1}, b \in U_{2}\right\}$ is equicontinuous $*$-regularising set and $\left\{R(a \# b) \mid a \in U_{1}, b \in U_{2}\right\}$ is bounded in $\Gamma_{A_{p}, \rho}^{\left(M_{p}\right), \infty}\left(\mathbb{R}^{2 d} ; m\right)$ for some $m>0$ (resp. of $\Gamma_{A_{p}, \rho}^{\left\{M_{p}\right\}, \infty}\left(\mathbb{R}^{2 d} ; h\right)$ for some $h>0$, cf. Lemma 2.1).

Hypoelliptic symbols have parametrices and hence they are globally regular; we can explicitly construct (the asymptotic expansions of) the parametrices.

Proposition 8.5 [17, Proposition 5.2] Let $a \in \Gamma_{A_{p}, \rho}^{*, \infty}\left(\mathbb{R}^{2 d}\right)$ be hypoelliptic. Define $q_{0}(w)=a(w)^{-1}$ on $Q_{B}^{c}$ and inductively, for $j \in \mathbb{Z}_{+}$,

$$
\begin{aligned}
q_{j}(x, \xi)= & -q_{0}(x, \xi) \sum_{s=1}^{j} \sum_{|\alpha+\beta|=s} \frac{(-1)^{|\beta|}}{\alpha ! \beta ! 2^{s}} \\
& \partial_{\xi}^{\alpha} D_{x}^{\beta} q_{j-s}(x, \xi) \partial_{\xi}^{\beta} D_{x}^{\alpha} a(x, \xi), \quad(x, \xi) \in Q_{B}^{c} .
\end{aligned}
$$

Then, for every $h>0$ there exists $C>0$ (resp. there exist $h, C>0)$ such that

$$
\left|D_{w}^{\alpha} q_{j}(w)\right| \leq C \frac{h^{|\alpha|+2 j} A_{|\alpha|+2 j}}{|a(w)|\langle w\rangle^{\rho(|\alpha|+2 j)}}, w \in Q_{B}^{c}, \alpha \in \mathbb{N}^{2 d}, j \in \mathbb{N} .
$$

If $B \leq 1$, then $\left(\sum_{j} q_{j}\right) \# a=1$ in $F S_{A_{p}, \rho}^{*, \infty}\left(\mathbb{R}^{2 d} ; 0\right)$. If $B>1$, one can extend $q_{0}$ to an element of $\Gamma_{A_{p}, \rho}^{*, \infty}\left(\mathbb{R}^{2 d}\right)$ by modifying it on $Q_{B^{\prime}} \backslash Q_{B}^{c}$, for $B^{\prime}>B$. In this case $\sum_{j} q_{j} \in F S_{A_{p}, \rho}^{*, \infty}\left(\mathbb{R}^{2 d} ; B^{\prime}\right),\left(\left(\sum_{j} q_{j}\right) \# a\right)_{k}=0$ on $Q_{B^{\prime}}^{c}, \forall k \in \mathbb{Z}_{+}$, and $\left(\left(\sum_{j} q_{j}\right) \# a\right)_{0}-$ $1=q_{0} a-1$ belongs to $\mathcal{D}^{\left(A_{p}\right)}\left(\mathbb{R}^{2 d}\right)\left(\right.$ resp. $\left.\mathcal{D}^{\left\{A_{p}\right\}}\left(\mathbb{R}^{2 d}\right)\right)$. 
In particular, for $q \sim \sum_{j} q_{j}$ there exists $*$-regularising operator $T$ such that $q^{w} a^{w}=\mathrm{Id}+T$.

Remark 8.6 A similar construction yields $\tilde{q} \in \Gamma_{A_{p}, \rho}^{*, \infty}\left(\mathbb{R}^{2 d}\right)$ such that $a^{w} \tilde{q}^{w}-$ Id is *-regularising (see [17, Subsection 6.2.1] for more details). Knowing this, it is easy to prove that we can use the left parametrix $q^{w}$ as a right one as well, i.e. both $q^{w} a^{w}-$ Id and $a^{w} q^{w}-$ Id are $*$-regularising.

Remark 8.7 For hypoelliptic $a \in \Gamma_{A_{p}, \rho}^{*, \infty}\left(\mathbb{R}^{2 d}\right)$, we can construct a parametrix $q$ out of $\sum_{j} q_{j} \in F S_{A_{p}, \rho}^{*, \infty}\left(\mathbb{R}^{2 d} ; B^{\prime}\right)$ in a specific way. Namely, applying Corollary 8.3 to $\left(\sum_{j} q_{j}\right) \# a$ together with (8.2) and Proposition 3.1, we conclude the existence of $R>0$ and a $*$-regularising operator $T$ such that $q^{w} a^{w}=\operatorname{Id}+T$, where $q=$ $R\left(\sum_{j} q_{j}\right) \in \Gamma_{A_{p}, \rho}^{*, \infty}\left(\mathbb{R}^{2 d}\right)$ satisfies the following conditions: there exist $B^{\prime \prime} \geq B^{\prime}$ and $c^{\prime \prime}, C^{\prime \prime}>0$ such that

$$
c^{\prime \prime} /|a(w)| \leq|q(w)| \leq C^{\prime \prime} /|a(w)|, \quad \forall w \in Q_{B^{\prime \prime}}^{c},
$$

and for every $h>0$ there exists $C>0$ (resp. there exist $h, C>0$ ) such that

$$
\left|D_{w}^{\alpha} q(w)\right| \leq C h^{|\alpha|} A_{\alpha}|a(w)|^{-1}\langle w\rangle^{-\rho|\alpha|}, w \in Q_{B^{\prime \prime}}^{c}, \alpha \in \mathbb{N}^{2 d}, j \in \mathbb{N} .
$$

In particular, $q$ is hypoelliptic. This estimate leads to the following simple observation. Assume that $a$ is hypoelliptic and $|a(w)| \rightarrow \infty$ as $|w| \rightarrow \infty$ and let $q$ be the parametrix for $a$ constructed above. Take $\psi \in \mathcal{D}^{\left(A_{p}\right)}\left(\mathbb{R}^{2 d}\right)$ (resp. $\psi \in \mathcal{D}^{\left\{A_{p}\right\}}\left(\mathbb{R}^{2 d}\right)$ ) such that $0 \leq \psi \leq 1, \psi=1$ on a compact neighbourhood of $Q_{B^{\prime \prime}}$ and $\psi=0$ on the complement of a slightly larger neighbourhood. Then, for each $n \in \mathbb{Z}_{+}$, the function $b_{n}(w)=q(w) \psi(w / n)$ is in $\mathcal{D}^{\left(A_{p}\right)}\left(\mathbb{R}^{2 d}\right)$ (resp. in $\mathcal{D}^{\left\{A_{p}\right\}}\left(\mathbb{R}^{2 d}\right)$ ) and hence $b_{n}^{w}$ is $*$-regularising for each $n \in \mathbb{Z}_{+}$. Employing the fact $|a(w)| \rightarrow \infty$ as $|w| \rightarrow \infty$ together with (8.4), one easily verifies that $b_{n} \rightarrow q$ in $\Gamma_{\rho}^{0}\left(\mathbb{R}^{2 d}\right)$ and hence $b_{n}^{w} \rightarrow q^{w}$ in $\mathcal{L}_{b}\left(L^{2}\left(\mathbb{R}^{d}\right), L^{2}\left(\mathbb{R}^{d}\right)\right.$ ) (see [13, Theorem 1.7.14, p. 58]). As $b_{n}^{w}, n \in \mathbb{Z}_{+}$, are compact operators on $L^{2}\left(\mathbb{R}^{d}\right)$, so is $q^{w}$.

\section{References}

1. Bingham, N.H., Goldie, C.M., Teugels, J.L.: Regular Variation, Encyclopedia of Mathematics and Its Applications, vol. 27. Cambridge University Press, Cambridge (1989)

2. Cappiello, M.: Pseudodifferential parametrices of infinite order for SG-hyperbolic problems. Rend. Sem. Mat. Univ. Pol. Torino 61(4), 411-441 (2003)

3. Cappiello, M.: Fourier integral operators of infinite order and applications to SG-hyperbolic equations. Tsukuba J. Math. 28, 311-361 (2004)

4. Cappiello, M., Pilipović, S., Prangoski, B.: Parametrices and hypoellipticity for pseudodifferential operators on spaces of tempered ultradistributions. J. Pseudo-Differ. Oper. Appl. 5(4), 491-506 (2014)

5. Cappiello, M., Pilipović, S., Prangoski, B.: Semilinear pseudodifferential equations in spaces of tempered ultradistributions. J. Math. Anal. Appl. 442(1), 317-338 (2016)

6. Carmichael, R., Kamiński, A., Pilipović, S.: Boundary Values and Convolution in Ultradistribution Spaces. World Scientific Publishing Co. Pte. Ltd., Singapore (2007)

7. Carracedo, C.M., Alix, M.S.: The Theory of Fractional Powers of Operators. Elsevier, Amsterdam (2001) 
8. Komatsu, H.: Ultradistributions, I: structure theorems and a characterization. J. Fac. Sci. Univ. Tokyo, Sect. IA Math. 20(1), 25-105 (1973)

9. Komatsu, H.: Ultradistributions, II: the kernel theorem and ultradistributions with support in a submanifold. J. Fac. Sci. Univ. Tokyo Sect. IA Math. 24(3), 607-628 (1977)

10. Komatsu, H.: Ultradistributions, III: vector valued ultradistributions and the theory of kernels. J. Fac. Sci. Univ. Tokyo Sect. IA Math. 29(3), 653-717 (1982)

11. Korevaar, J.: Tauberian Theory. A Century of Developments. Springer, Berlin (2004)

12. Köthe, G.: Topological Vector Spaces II. Springer, New York (1979)

13. Nicola, F., Rodino, L.: Global Psedo-Differential Calculus on Euclidean Spaces. Birkhäuser, Basel (2010)

14. Pazy, A.: Semigroups of Linear Operators and Applications to Partial Differential Equations. Springer, New York (1983)

15. Pilipović, S.: Tempered ultradistributions. Boll. Unione Mat. Ital. 2(2), 235-251 (1988)

16. Pilipović, S., Prangoski, B.: Anti-Wick and Weyl quantization on ultradistribution spaces. J. Math. Pures Appl. 103(2), 472-503 (2015)

17. Pilipović, S., Prangoski, B.: Complex powers for a class of infinite order hypoelliptic operators. to appear in Dissertationes Mathematicae. (2018). arXiv:1609.03728

18. Prangoski, B.: Pseudodifferential operators of infinite order in spaces of tempered ultradistributions. J. Pseudo-Differ. Oper. Appl. 4(4), 495-549 (2013)

19. Schaefer, H.H.: Topological Vector Spaces. Springer, Berlin (1970)

20. Shubin, M.A.: Pseudodifferential Operators and Spectral Theory. Springer, Berlin (2001)

\section{Publisher's Note}

Springer Nature remains neutral with regard to jurisdictional claims in published maps and institutional affiliations. 